\title{
Stereoselective synthesis of trans-dihydronarciclasine derivatives containing a 1,4-benzodioxane moiety
}

\author{
Gábor Varró $^{1,2} \cdot$ Balázs Pogrányi ${ }^{1} \cdot$ Alajos Grün ${ }^{1} \cdot$ András Simon $^{3} \cdot$ László Hegedús $^{1} \cdot$ István Kádas $^{1}$
}

Received: 27 June 2018 / Accepted: 3 September 2018/ Published online: 26 October 2018

(C) Springer-Verlag GmbH Austria, part of Springer Nature 2018

\begin{abstract}
Some new trans-dihydronarciclasine derivatives containing a 1,4-benzodioxane moiety were stereoselectively synthesised using our feasible and efficient method developed recently. These new phenanthridone alkaloid analogues were obtained in both racemic and optically active forms. High enantioselectivities (up to 99\% ee) were achieved by applying (8S,9S)-9amino(9-deoxy)epiquinine as an organocatalyst. Due to a side reaction, various methoxyphenanthridine regioisomers were also prepared which afforded further synthetic trans-dihydronarciclasine analogues modified in the ring A of the phenanthridone scaffold.
\end{abstract}

\section{Graphical abstract}

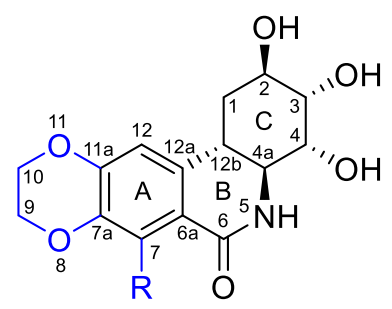

8: $\mathrm{R}=\mathrm{H}$

9: $\mathrm{R}=\mathrm{OH}$

10: $\mathrm{R}=\mathrm{OMe}$<smiles>COc1cc2c(c3c1OCCO3)C(=O)N[C@@H]1[C@H]2C[C@H](O)[C@@H](O)[C@@H]1O</smiles>

11

Keywords Alkaloids · Antitumor agents · Heterocycles · Organocatalysis · Total synthesis

Electronic supplementary material The online version of this article (https://doi.org/10.1007/s00706-018-2287-7) contains supplementary material, which is available to authorized users.

István Kádas

ikadas@mail.bme.hu

1 Department of Organic Chemistry and Technology, Budapest University of Technology and Economics, Budafoki út 8, Budapest 1111, Hungary

2 Gedeon Richter Plc., Gyömrői út 19-21, Budapest 1103, Hungary

3 Department of Inorganic and Analytical Chemistry, Budapest University of Technology and Economics, Szt. Gellért tér 4, Budapest 1111, Hungary

\section{Introduction}

Nowadays, hundreds of members of alkaloids are known, which have been isolated from the plants of Amaryllidaceae family. After recognising their anticancerous properties, more and more attention has been paid to their isolation and structural elucidation [1]. The alkaloids prepared from this plant family are divided into 12 groups according to their ring systems [2]. Among these diverse structures, the most potent antitumorous alkaloids can be found in the phenanthridone subgroup. Surprisingly, the detailed investigations of Amaryllidaceae alkaloids started only at the end of the nineteenth century, when Gerrard isolated lycorine (1, Fig. 1) from Narcissus 
<smiles>OC1C=C2CCN3Cc4cc5c(cc4[C@@H]([C@H]1O)[C@H]23)OCO5</smiles><smiles>[R]c1c2c(cc3c1C(=O)N[C@H]1C[C@H]3C[C@@H](O)[C@@H](O)[C@@H]1O)OCO2</smiles>

1: lycorine

2: $\mathrm{R}=\mathrm{OH}$ trans-dihydronarciclasine 3: R=H 7-deoxy-trans-dihydronarciclasine<smiles>[R]c1c2c(cc3c1C(=O)N[C@@H]1[C@@H](O)[C@H](O)[C@H](O)[C@H](O)[C@H]31)OCO2</smiles><smiles>[R]c1c2c(cc3c1C(=O)N[C@H]1C3=C[C@@H](O)C(O)C(O)[C@@H]1O)OCO2</smiles>

4: $\mathrm{R}=\mathrm{OH}$ pancratistatin 5: $\mathrm{R}=\mathrm{H} \quad$ 7-deoxypancratistatin

6: $\mathrm{R}=\mathrm{OH}$ narciclasine 7: $\mathrm{R}=\mathrm{H} \quad$ lycoricidine

Fig. 1 Structures of the most important Amaryllidaceae alkaloids (1-7)

pseudonarcissus in 1877 [3, 4]. Since then, several alkaloids have been obtained from various Amaryllidaceae plants including trans-dihydronarciclasine (2, Fig. 1) isolated by Pettit and co-workers from Zephyranthes candida in 1990 [5].

Compound $\mathbf{2}$ is the most potent natural anticancerous alkaloid among the Amaryllidaceae ones according to the data from National Cancer Institute (NCI, USA) [6]. Its 7-deoxy analogue (3, Fig. 1) was also isolated by Pettit and co-workers from Hymenocallis caribaea and Hymenocallis latifolia, in pure form, but its biological activity was found to be weaker than that of 2 [6]. This can be explained by the lack of hydroxyl group at the position A-7, similarly to the comparison of other representatives, such as pancratistatin (4) and 7-deoxypancratistatin (5) or narciclasine (6) and lycoricidine (7) (Fig. 1) [6]. Further structure-activity relationship studies have intensively been made to find more effective synthetic analogues, but the modifications rather touched the ring $\mathrm{C}$ of the phenanthridone skeleton [7-20] than its ring A [21-28].

Furthermore, the Amaryllidaceae alkaloids also showed significant antiviral effects. Thus, compound $\mathbf{1}$ has strong activity against herpes simplex 1 and varicella zoster DNA viruses [29] and against several RNA viruses, such as avian influenza virus (H5N1) [30] or SARS coronavirus [31]. It has also inhibitory effects against reverse transcriptase enzyme in the HIV-1 virus [32]. Besides, strong antiviral activities of some phenanthridone alkaloids including compounds 4, 6, and 7 were reported by Gabrielsen and coworkers [33, 34]. Very recently, trans-dihydronarciclasine, pancratistatin, and narciclasine have also been found to be active against Zika virus [35].

Previously, the racemic [36] and the ent-forms of 3 [37], as well as those of $\boldsymbol{2}$ [38, 39], were stereoselectively
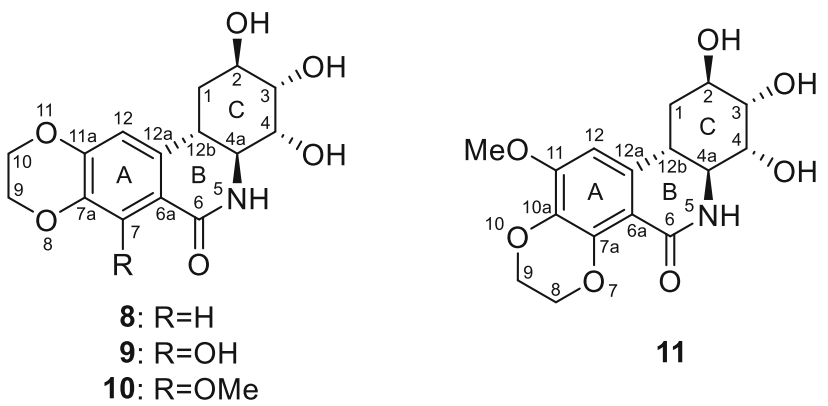

10: $\mathrm{R}=\mathrm{OMe}$

11

Fig. 2 Structures of trans-dihydronarciclasine derivatives containing a 1,4-benzodioxane moiety (8-11)

synthesised using our facile and efficient process developed recently. Later, we also reported the highly stereoselective synthesis of a series of analogues of $\mathbf{2}$ substituted by alkyloxy groups (ethoxy and/or methoxy) in the aromatic ring [40]. In this work, focusing further on the modification of ring $\mathrm{A}$ of the phenanthridone scaffold by introducing a relatively rigid substituent, the stereoselective syntheses of some new trans-dihydronarciclasine analogues containing a 1,4-benzodioxane moiety (Fig. 2), such as 2,3,4-trihydroxy-1,3,4,4a,5,9,10,12b-octahydro[1,4]dioxino[2,3-j]phenanthridin-6(2H)-one $(\mathbf{8}), \quad 2,3,4,7$-tetrahydroxy1,3,4,4a,5,9,10,12b-octahydro[1,4]dioxino[2,3-j]phenanthridin-6(2H)-one (9), 2,3,4-trihydroxy-7-methoxy-1,3,4,4a,5,9,10,12b-octahydro[1,4]dioxino[2,3-

$j]$ phenanthridin-6(2H)-one (10) and 2,3,4-trihydroxy-11methoxy-1,2,3,4,4a,8,9,12b-octahydro[1,4]dioxino[2,3$i$ phenanthridin-6(2H)-one (11), were described in both racemic and enantiopure forms.

Our modifying strategy related to ring A of the phenanthridone skeleton aimed at the methylenedioxy ($\mathrm{OCH}_{2} \mathrm{O}_{-}$) structural part, a rigid functional group, in transdihydronarciclasine, because this molecule part may also be responsible for the anticancerous activity of this alkaloid. Thus, the homologous ethylenedioxy $\left(-\mathrm{OCH}_{2} \mathrm{CH}_{2} \mathrm{O}-\right)$ one was introduced into the aromatic ring to obtain new analogues for further structure-activity relationship studies.

\section{Results and discussion}

Similarly to our previous synthetic works [38-40], the starting material was vanillin (12), an inexpensive and readily available substance. As seen in Scheme 1 , in the initial step, compound $\mathbf{1 2}$ was demethylated to 3,4-dihydroxybenzaldehyde (13) in dichloromethane by $\mathrm{AlCl}_{3}$ and pyridine, using a known method [41], in good yield (92\%). Hydroxyvanillin (15) was also obtained from 12, but in this case, at first, it was selectively iodinated to 5-iodovanillin (14) [42] almost quantitatively, and then compound $\mathbf{1 4}$ was 


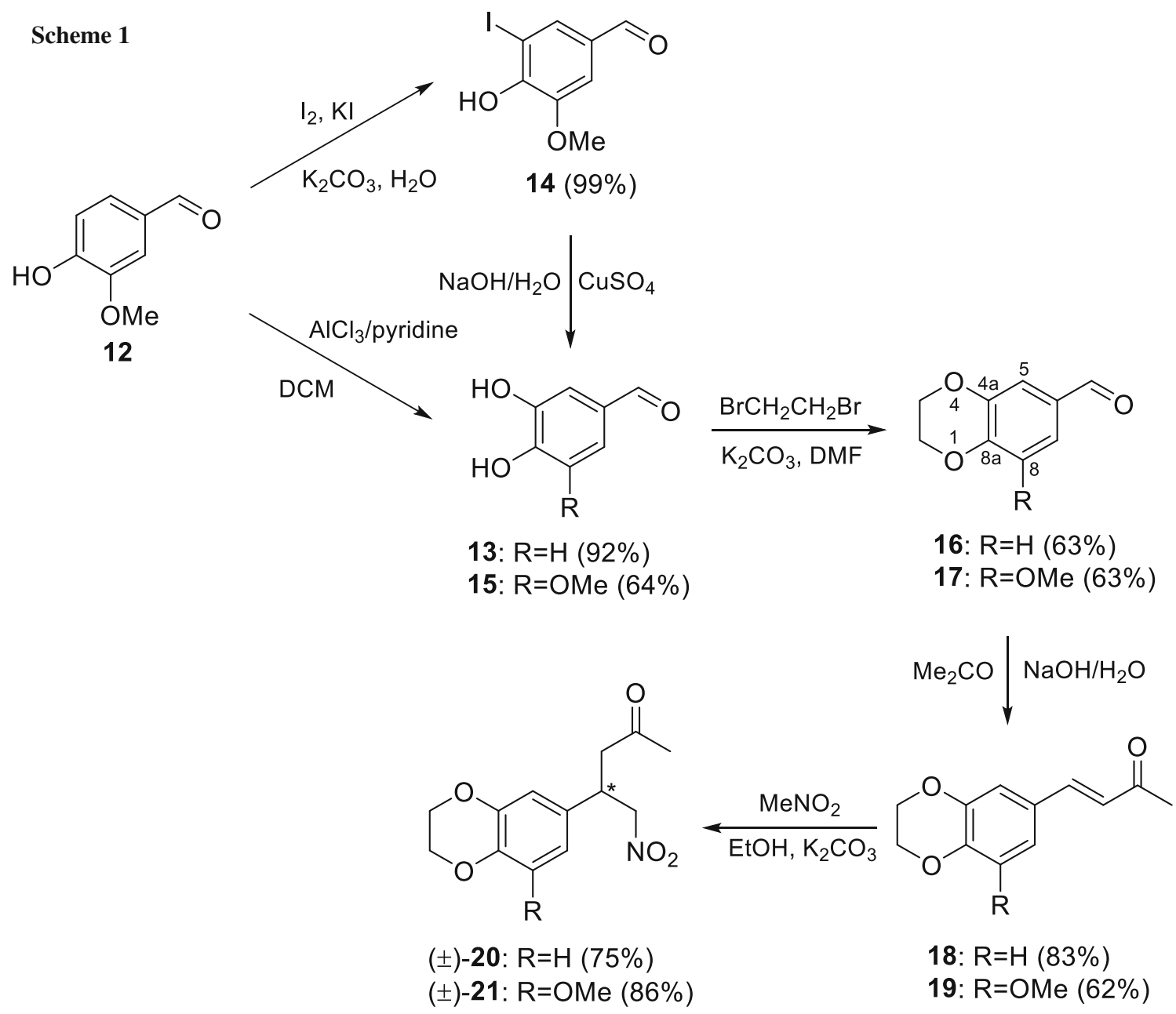

hydrolysed with $20 \%$ aqueous $\mathrm{NaOH}$ solution in presence of $\mathrm{CuSO}_{4}$ [43] to give $\mathbf{1 5}$ in $64 \%$ yield. The dihydrobenzodioxine ring was formed with 1,2-dibromoethane in DMF, in the presence of $\mathrm{K}_{2} \mathrm{CO}_{3}$ [44] to afford 2,3-dihydrobenzo[b][1,4]dioxine-6-carbaldehyde (16) and 8-methoxy-2,3- dihydrobenzo[ $b][1,4]$ dioxine-6-carbaldehyde (17) in good yield $(63 \%)$. In the next step, acetone was condensed with these benzaldehyde derivatives $\mathbf{1 6}$ and $\mathbf{1 7}$ in the Claisen-Schmidt reaction applying a high excess of acetone in water and basic conditions $(\mathrm{NaOH})$ to give 4-(2,3-dihydrobenzo[b][1,4]dioxin-6-yl)but-3-en-2-one

(18) and 4-(8-methoxy-2,3-dihydrobenzo[b][1,4]dioxin-6yl)but-3-en-2-one (19) in good yields (83 and 62\%, respectively). Since dibenzylidene acetone derivatives may also be formed during this reaction, the pure products were obtained after distillation.

The first asymmetric centre of the title molecules was formed by Michael addition of nitromethane to compounds 18 and 19 to afford ( \pm )-4-(2,3-dihydrobenzo[b][1,4]dioxin-6-yl)-5-nitropentan-2-one $[( \pm)-20]$ and $( \pm)-4-(8-$ methoxy-2,3-dihydrobenzo[b][1,4]dioxin-6-yl)-5-nitropentan-2-one $[( \pm)-21]$. These racemic representatives were prepared by using Peseke and co-workers' mild method $\left(\mathrm{MeNO}_{2}, \mathrm{EtOH}, \mathrm{K}_{2} \mathrm{CO}_{3}\right)$ [45] in very good yields (75-86\%). Moreover, this reaction allows an opportunity to obtain these intermediates in enantiomerically pure form.

The results of the enantioselective Michael addition are summarised in Table 1. Using $20 \mathrm{~mol} \%$ (8S,9S)-9amino(9-deoxy)epiquinine (22) organocatalyst [46-53] under conditions applied previously [39, 40], the optically active nitropentanones (-)-20 and (-)-21 were prepared in good yield (67\%) and excellent enantioselectivity (92-98\% $e e)$ after 7 days. The negative optical rotation values suggest, in accordance with our previous studies [39, 40], that these new intermediates enable synthesising further entforms of natural alkaloid analogues.

Henceforth, only the syntheses of the optically active compounds are shown, but the racemic derivatives can be afforded in the same way. As seen in Scheme 2, the ring C was built by the Claisen-Henry reaction using ethyl 
Table 1 Enantioselective Michael addition of nitromethane to $\mathbf{1 8}$ and $\mathbf{1 9}$ catalysed by (8S,9S)-9-amino(9-deoxy)epiquinine (22)

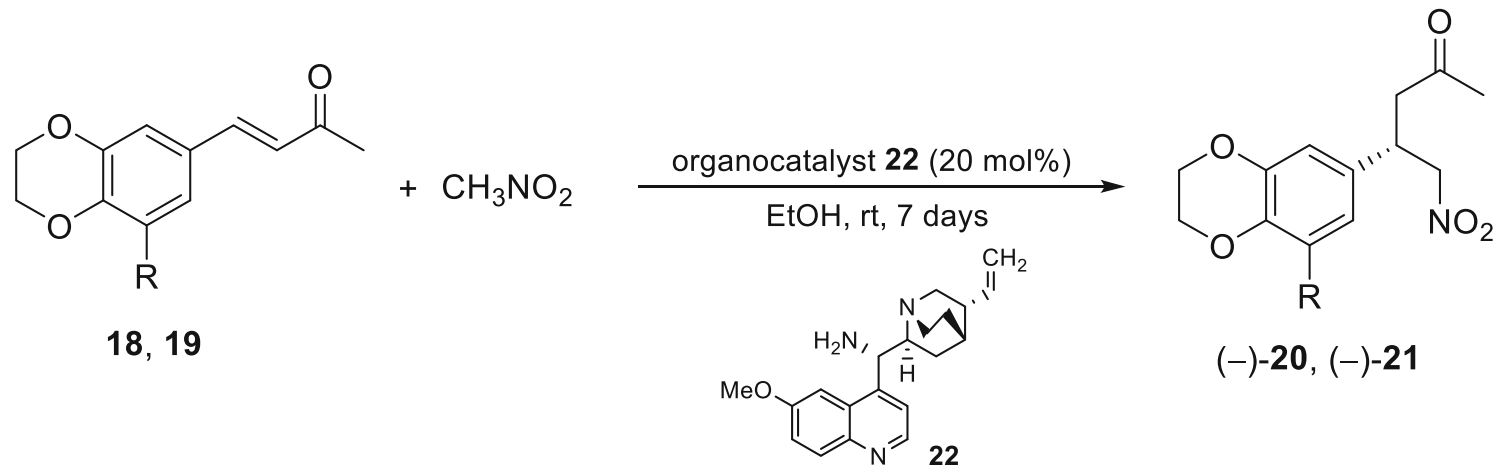

\begin{tabular}{lllll}
\hline Entry & $\mathrm{R}$ & Product & ${\text { Yield } / \%^{\mathrm{a}}}^{\mathrm{a}}$ & $e e / \%^{\mathrm{b}}$ \\
\hline 1 & $\mathrm{H}$ & $(-)-\mathbf{2 0}$ & 67 & 92 \\
2 & $\mathrm{OMe}$ & $(-)-\mathbf{2 1}$ & 67 & 98 \\
\hline
\end{tabular}

${ }^{\mathrm{a}}$ Isolated yield

${ }^{\mathrm{b}}$ Determined by chiral HPLC

formate and dry sodium methoxide in anhydrous diethyl ether to give (-)-3-hydroxy-5- $\left(2^{\prime}, 3^{\prime}\right.$-dihydrobenzo$[b][1,4]$ dioxin-6 $-y 1)-4-n i t r o c y c l o h e x a n o n e ~[(-)-23]$ and (-)-3-hydroxy-5-(8'-methoxy-2',3'-dihydrobenzo[b][1,4]dioxin-6'-yl)-4-nitrocyclohexanone $[(-)-24]$ in moderate yields (46-57\%) after column chromatography or recrystallisation from ethyl acetate. Full diastereoselectivity was achieved in this cyclisation step which can be explained by the H-bond formation between the C-3 hydroxy and C-4 nitro groups (Fig. 3), as described by Walker [54] and observed by us previously [36-40]. Prior to the reduction of the nitro group into the amino one, the carbonyl group of (-)-23 and (-)-24 was protected with ethylene glycol, in the presence of oxalic acid, in anhydrous acetonitrile to afford (+)-3-hydroxy-5-(2',3'-dihydrobenzo[b][1,4]dioxin-

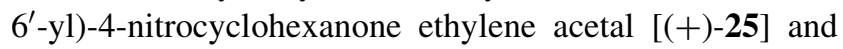
(+)-3-hydroxy-5-(8'-methoxy-2',3'-dihydrobenzo[b][1,4]dioxin- $\left.6^{\prime}-y 1\right)-4$-nitrocyclohexanone ethylene acetal $[(+)-$ 26] in good yields (75-84\%). Compound (+)-25 or (+)-26 was converted to the corresponding amino derivatives, such as (+)-4-amino-3-hydroxy-5-( $2^{\prime}, 3^{\prime}$-dihydrobenzo[ $\left.b\right]$ $[1,4]$ dioxin- $\left.6^{\prime}-y 1\right)$ cyclohexanone ethylene acetal [(+)-27] and (+)-4-amino-3-hydroxy-5-( $8^{\prime}$-methoxy-2', $3^{\prime}$-dihydrobenzo $[b][1,4]$ dioxin- $\left.6^{\prime}-y l\right) c y c l o h e x a n o n e \quad$ ethylene acetal $[(+)-28]$, in good yields $(56-75 \%)$ using our catalytic hydrogenation method $[39,55]: 10 \% \mathrm{Pd} / \mathrm{C}$ (Selcat Q [56]), methanol, $60-80{ }^{\circ} \mathrm{C}$ and 12 bar. Subsequently, amino ketal $(+)-27$ or $(+)-28$ was reacted with methyl chloroformate in a biphasic solvent mixture (water and THF) to obtain (+)-3-hydroxy-4-methoxycarbonylamino5 - $\left(2^{\prime}, 3^{\prime}\right.$-dihydrobenzo $[b][1,4]$ dioxin- 6 -yl $)$ cyclohexanone ethylene acetal $[(+)-29]$ or $(+)-3$-hydroxy-4- methoxycarbonylamino-5-( $8^{\prime}$-methoxy-2',3'-dihydrobenzo[b][1,4]dioxin- $6^{\prime}$-yl)cyclohexanone ethylene acetal [(+)-30] quantitatively. The ketal protective group of $(+)-29$ or $(+)-30$ was removed in acetone containing a catalytic amount of $p$ toluenesulphonic acid under reflux, but water elimination also took place to afford (-)-6-(2',3'-dihydrobenzo[ $b][1,4]$ dioxin$6^{\prime}$-yl)-4-oxocyclohex-2-enyl)carbamic acid methyl ester [(-)31] or (-)-6- $\left(8^{\prime}\right.$-methoxy- $2^{\prime}, 3^{\prime}$-dihydrobenzo[ $\left.b\right][1,4]$ dioxin$6^{\prime}$-yl)-4-oxocyclohex-2-enyl)carbamic acid methyl ester [(-)32] in excellent yields (88-93\%).

Then the oxo group of (-)-31 or (-)-32 was stereoselectively reduced with $\mathrm{NaBH}_{4}$, in the presence of $\mathrm{CaCl}_{2}$, in methanol (Utimoto's method [57]) to obtain (-)-6-(2',3'dihydrobenzo[b][1,4]dioxin-6'-yl)-4-hydroxycyclohex-2enyl)carbamic acid methyl ester $[(-)-33]$ or $(-)-6-\left(8^{\prime}-\right.$ methoxy-2 $2^{\prime}, 3^{\prime}$-dihydrobenzo[ $\left.b\right][1,4]$ dioxin-6'-yl)-4-hydroxycyclohex-2-enyl)carbamic acid methyl ester [(-)-34] in good yields $(67-79 \%)$ and excellent enantiopurity ( $>99 \% e e$ ) after recrystallisation from hexane/ethyl acetate $(2: 1)$. This very high stereoselectivity was achieved with an axial attack of the small hydride ion derived from $\mathrm{NaBH}_{4}$ enhanced by the coordination with $\mathrm{Ca}^{2+}$, resulting in an equatorial position of the hydroxy group formed newly (Scheme 3). However, the structure of the title compounds requires the inversion of this asymmetry centre. For this purpose, the Mitsunobu reaction [58] (triphenylphosphine, diethyl azodicarboxylate, anhydrous THF) seemed to be an obvious choice. Thus, compound (-)-33 or (-)-34 was converted to (+)-5-(2',3'-dihydrobenzo[b][1,4]dioxin-6'-yl)-4-(methoxycarbonylamino)cyclohex2-enyl benzoate $[(+)-35]$ and $(+)-5-\left(8^{\prime}\right.$-methoxy-2',3'-dihydrobenzo $[b][1,4]$ dioxin- $\left.6^{\prime}-y 1\right)-4-($ methoxycarbonylamino)- 


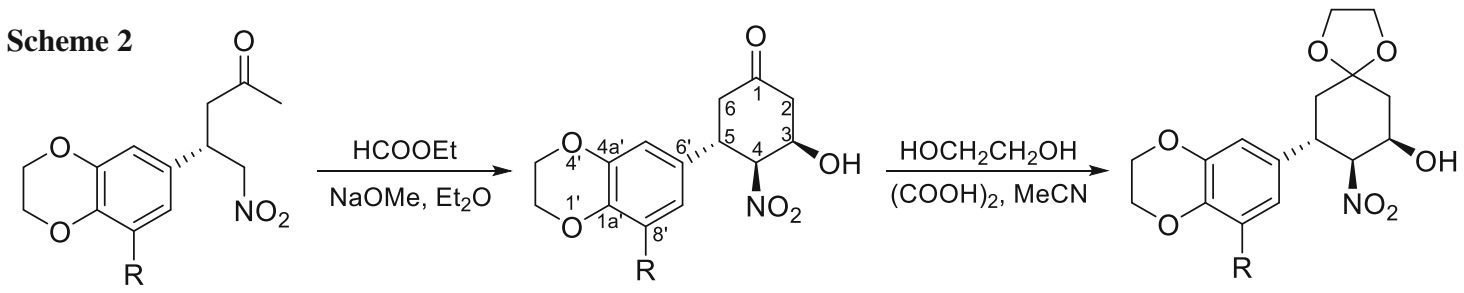
$(-)-20 \mathrm{R}=\mathrm{H}$
$(-)-23 \mathrm{R}=\mathrm{H}(46 \%)$
$(-)-21 \mathrm{R}=\mathrm{OMe}$
$(-)-24 \mathrm{R}=\mathrm{OMe}(57 \%)$

(+)-25 R=H $(84 \%)$

$(+)-26 \mathrm{R}=\mathrm{OMe}(75 \%)$

$\mathrm{H}_{2}, 10 \% \mathrm{Pd} / \mathrm{C}$<smiles>[R]c1cc([C@H]2CC(=O)C=C[C@@H]2NC(=O)OC)cc2c1OCCO2</smiles>

$(-)-31 \mathrm{R}=\mathrm{H}(93 \%)$

$(-)-32 \mathrm{R}=\mathrm{OMe}(88 \%)$<smiles>[R]c1cc([C@@H]2CCC[C@@H](O)[C@H]2NC(=O)OC)cc2c1OCC(CC(C)=O)O2</smiles>

(+)-29 R=H (100\%)

(+)-30 R=OMe $(99 \%)$<smiles>[R]c1cc([C@@H]2CC3(C[C@H](O)[C@@H]2N)OCCO3)cc2c1OCCO2</smiles>

(+)-27 R=H (56\%)

(+)-28 R=OMe $(75 \%)$

$\mathrm{NaBH}_{4}, \mathrm{CaCl}_{2} \downarrow \mathrm{MeOH}$<smiles>[R]c1cc([C@H]2C[C@@H](O)CC[C@@H]2NC(=O)OC)cc2c1OCCO2</smiles>

(-)-33 R=H (66\%, ee $99 \%)$

$(-)-34 \mathrm{R}=$ OMe $(79 \%$, ee $99 \%)$
(+)-35 R=H $(77 \%)$

(+)-36 R=OMe $(77 \%)$
$(+)-37 \mathrm{R}=\mathrm{H}(99 \%)$

(+)-38 R=OMe $(100 \%)$

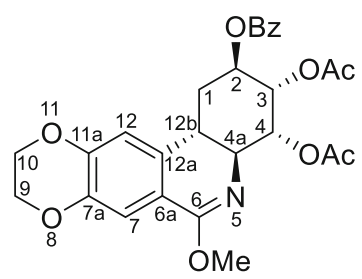

$(-)-41(20 \%)$<smiles>COC1=N[C@H]2[C@@H](C[C@H](OC(C)=O)[C@H](OC(C)=O)[C@@H]2OC(C)=O)c2cc(OC)c3c(c21)OCCO3</smiles>

(-)-43 (87\%)

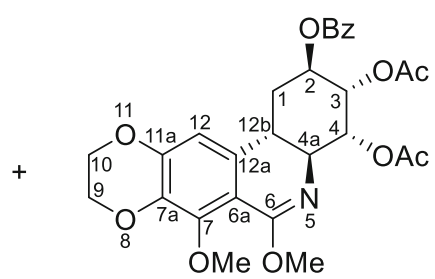

$(-)-42(87 \%)$

inseparable regioisomers (1:1) 


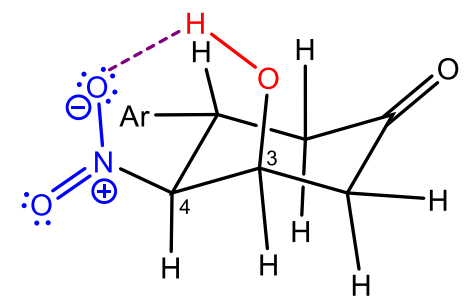

(-)-23 $\mathrm{Ar}=2^{\prime}, 3^{\prime}$-dihydrobenzo[b][1,4]dioxin-6'-yl

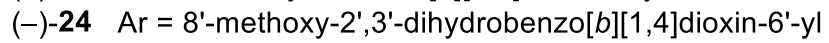

Fig. 3 Presumed structure of the 3-hydroxy-4-nitrocyclohexanone derivatives [(-)-23 or (-)-24] and its stabilisation by hydrogen bonding

cyclohex-2-enyl benzoate [(+)-36] using benzoic acid, under Mitsunobu conditions, in good yields (77\%) after column chromatography. In the next step, the stereoselective attack of osmium tetroxide to the $\mathrm{C}=\mathrm{C}$ bond of $(+)-\mathbf{3 5}$ or (+)-36, adapting the Sharpless-Upjohn method [59] $\left(\mathrm{OsO}_{4}, \mathrm{~N}\right.$-methyl-morpholine $\mathrm{N}$-oxide, THF- $\left.\mathrm{H}_{2} \mathrm{O}\right)$, was favoured by the steric hindrance of the bulky benzoyl group in axial position resulting in cis-diols, such as (+)-5( $2^{\prime}, 3^{\prime}$-dihydrobenzo[b][1,4]dioxin-6'-yl)-2,3-dihydroxy-4(methoxycarbonylamino)cyclohexyl benzoate $[(+)-37]$ and (+)-2,3-dihydroxy-5-( $8^{\prime}$-methoxy-2', 3'-dihydrobenzo $[b]$ $[1,4]$ dioxin-6'-yl)-4-(methoxycarbonylamino)cyclohexyl benzoate $[(+)-38]$, in excellent yields $(99-100 \%)$. Prior to the Bischler-Napieralski cyclisation modified by Banwell and co-workers [60], the hydroxy groups of $(+)-$ 37 or (+)-38 were protected by acetyl chloride to afford (-)-2,3-diacetoxy-5-(2',3'-dihydrobenzo[b][1,4]dioxin$6^{\prime}$-yl)-4-(methoxycarbonylamino)cyclohexyl benzoate [(-)-39] and (-)-2,3-diacetoxy-5-( $8^{\prime}$-methoxy-2', $3^{\prime}$-dihydrobenzo[1,4]dioxin-6'-yl)-4-(methoxycarbonylamino)cyclohexyl benzoate [(-)-40] in excellent yields (81-100\%). The ring closure reaction was performed with trifluoromethanesulphonic anhydride $\left(\mathrm{Tf}_{2} \mathrm{O}\right)$ and 4-(dimethylamino)pyridine (DMAP) in dichloromethane to give (-)-2benzoyloxy-6-methoxy-1,2,3,4,4a,9,10,12b-octahydro[1,4]dioxino-[2,3-j]phenanthridin-3,4-diyl diacetate $[(-)-41]$ in $20 \%$ yield, as well as an inseparable mixture of (-)-2-benzoyloxy-6,7-dimethoxy-1,2,3,4,4a,9,10,12b-octahydro[1,4]dioxino[2,3-j]phenanthridin-3,4-diyl diacetate $[(-)-\mathbf{4 2}]$ and (-)-2-benzoyloxy-6,11-dimethoxy-1,2,3,4,4a, 8,9,12b-octahydro[1,4]dioxino[2,3-i]phenanthridin-3,4-diyl diacetate [(-)43 ] in a ratio of $1: 1$, in very good yield (87\%). Although the $8^{\prime}$-deoxy derivative (-)-39 gave selectively compound (-)41, it was converted spontaneously into the corresponding lactam one resulting in its poor yield.

As seen in Scheme 4, the acidic treatment of (-)-41 with $2 \mathrm{M} \mathrm{HCl}$ in THF afforded (-)-2-benzoyloxy-6-oxo$1,2,3,4,4 \mathrm{a}, 9,10,12 \mathrm{~b}$-octahydro[1,4]dioxino[2,3-j]phenanthridine-3,4-diyl diacetate $[(-)-44]$ in moderate yield (54\%), which was subsequently deacylated by the Zemplén's method [61] $(\mathrm{NaOMe} / \mathrm{MeOH})$ in THF to give the title compound $(-)-\mathbf{8}$ in good yield $(71 \%)$.

In the next reactions the racemic form of $( \pm)-\mathbf{4 2}$ and $( \pm)-\mathbf{4 3}$ was used, but the same results could be obtained by applying their optically active ones. When the 1:1 mixture of regioisomers $( \pm)-\mathbf{4 2}$ and $( \pm)-\mathbf{4 3}$ was quantitatively converted into lactams, such as ( \pm )-2-benzoyloxy-7methoxy-6-oxo-1,2,3,4,4a,8,9,12b-octahydro[1,4]dioxino[2,3-j]phenanthridine-3,4-diyl diacetate $[( \pm)-45]$ and $( \pm)$-2-benzoyloxy-11-methoxy-6-oxo-1,2,3,4,4a,8,9,12boctahydro[1,4]dioxino[2,3-i]phenanthridine-3,4-diyl diacetate $[( \pm)-46]$, in the same way (Scheme 5), the methyl group of 7-methoxy derivative ( \pm -45 was selectively cleaved with iodotrimethylsilane (TMS-I) prepared in situ from chlorotrimethylsilane (TMS-Cl) and potassium iodide, in anhydrous acetonitrile to obtain $( \pm)$-2-benzoyloxy-7-hydroxy-6-oxo-1,2,3,4,4a,9,10,12b-octahydro[1,4]dioxino[2,3-j]phenanthridine-3,4-diyl diacetate [( \pm$)-47]$,
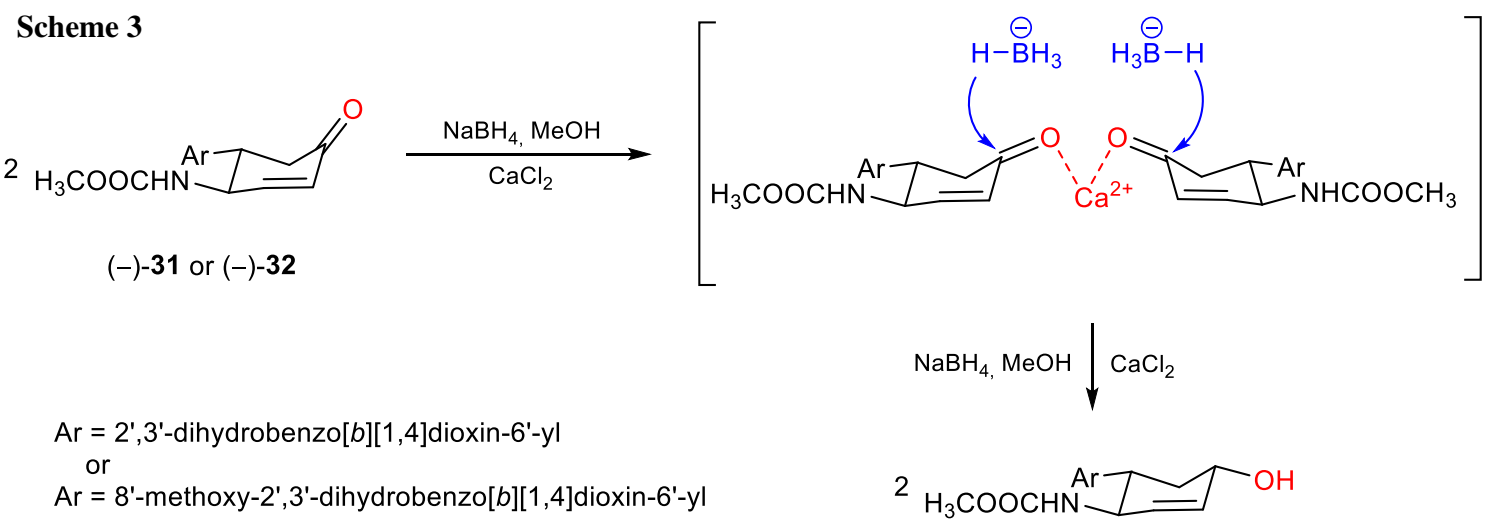

$(-)-33$ or $(-)-34$ 


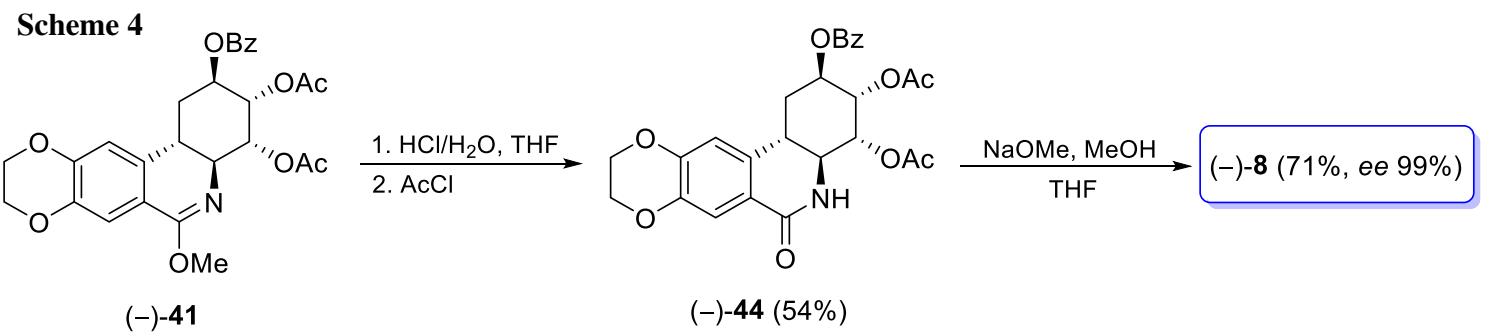

Scheme 5

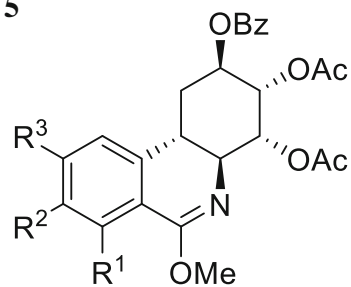
$\underset{\text { 1. } \mathrm{HCl} / \mathrm{H}_{2} \mathrm{O}, \mathrm{THF}}{\stackrel{\mathrm{AcCl}}{\longrightarrow}}$

$( \pm)-42 \mathrm{R}^{1}=\mathrm{OMe}, \mathrm{R}^{2}$ and $\mathrm{R}^{3}=\mathrm{O}\left(\mathrm{CH}_{2}\right)_{2} \mathrm{O}$ and $( \pm)-43 \mathrm{R}^{1}$ and $\mathrm{R}^{2}=\mathrm{O}\left(\mathrm{CH}_{2}\right)_{2} \mathrm{O}, \mathrm{R}^{3}=\mathrm{OMe}$<smiles>[R]c1cc2c(c([R])c1[R])C(=O)N[C@H]1[C@@H]2[C@H](OC(C)=O)[C@@H](OC(C)=O)[C@@H]1OC(C)=O</smiles>

$( \pm)-45 \mathrm{R}^{1}=\mathrm{OMe}, \mathrm{R}^{2}$ and $\mathrm{R}^{3}=\mathrm{O}\left(\mathrm{CH}_{2}\right)_{2} \mathrm{O}$ and $(100 \%)$ TMS-Cl, KI MeCN<smiles>CC(=O)O[C@H]1[C@@H](OC(C)(C)C)C[C@H]2c3cc4c(c(O)c3C(=O)N[C@@H]21)OCCO4</smiles>
$( \pm)-47(20 \%)$
$( \pm)-\mathbf{4 8}(8 \%)$

$( \pm)-49(13 \%)$

while the methyl group in A-11 position of another regioisomer $( \pm)-\mathbf{4 6}$ remained untouched allowing their separation. However, due to the poor yield (20\%) of $( \pm)-47$ and the formation of further two by-products, such as $( \pm)$ 3-acetamido-6-benzoyloxy-4-( $8^{\prime}$-methoxy-7'-methoxycarbonyl-2,3-dihydrobenzo[b][1,4]dioxin- $6^{\prime}$-yl)cyclohexane1,2-diyl diacetate $[( \pm)-48]$ and $( \pm)$-3-acetamido-6-benzoyloxy-4-(8'-methoxy-5'- methoxycarbonyl-2,3-dihydrobenzo[b][1,4]dioxin- $6^{\prime}$-yl)cyclohexane-1,2-diyl diacetate $[( \pm)-49]$, which proved also to be regioisomers (Fig. 4), our synthesis strategy was modified. The structures of these regioisomers were distinguished by the chemical shifts of $5^{\prime}-\mathrm{H}_{\mathrm{Ar}}$ and $5^{\prime}-\mathrm{C}_{\mathrm{Ar}}$ of $( \pm)-\mathbf{4 8}$, as well as $7^{\prime}-\mathrm{H}_{\mathrm{Ar}}$ and $7^{\prime}-\mathrm{C}_{\mathrm{Ar}}$ of $( \pm)-\mathbf{4 9}$, because there were significant differences between the positions of these peaks both in the ${ }^{1} \mathrm{H}$ NMR (6.72 ppm for $5^{\prime}-\mathrm{H}_{\mathrm{Ar}}$ and $6.54 \mathrm{ppm}$ for $7^{\prime}-\mathrm{H}_{\mathrm{Ar}}$ ) and ${ }^{13} \mathrm{C}$ NMR spectra (110.8 ppm for $5^{\prime}-\mathrm{C}_{\mathrm{Ar}}$ and $102.0 \mathrm{ppm}$ for $7^{\prime}-\mathrm{C}_{\mathrm{Ar}}$ ). These diversions were due to the anisotropic shielding effect of the adjacent methoxy group in compound $( \pm)-49$, which resulted in lower chemical shifts for those aromatic hydrogen and carbon atoms at position A-7.

At first, as shown in Scheme 6, the 1:1 mixture of regioisomers (-)-42 and (-)-43 was also deacylated using the Zemplén's method resulting in (-)-6,7-dimethoxy$1,2,3,4,4 \mathrm{a}, 9,10,12 \mathrm{~b}$-octahydro[1,4]dioxino[2,3-j]phenanthridine-2,3,4-triol [(-)-50] and (-)-6,11-dimethoxy$1,2,3,4,4 \mathrm{a}, 9,10,12 \mathrm{~b}$-octahydro[1,4]dioxino[2,3-i]phenanthridine-2,3,4-triol [(-)-51] in pure forms, in moderate yields (22-23\%) after column chromatography (ethyl acetate-ethanol, 20:1). After peracetylation of (-)-50 and (-)-51, the afforded (-)-7-methoxy-6-oxo-1,2,3,4,4a,9,10,12 b-octahydro[1,4]dioxino-[2,3-j]phenanthridine-2,3,- 
<smiles>CCCOC1C[C@H](c2cc3c(c(OC)c2C(=O)OC)OCCO3)C(NC(C)=O)[C@@H](OC(C)=O)[C@H]1OC(C)=O</smiles>

$( \pm)-48$<smiles>CCOC(C)=O</smiles>

$( \pm)-49$
Fig. 4 Structures of the isolated side products $( \pm)-\mathbf{4 8}$ and $( \pm)-\mathbf{4 9}$

4-triyl triacetate [(-)-52] and (-)-11-methoxy-6-oxo1,2,3,4,4a, 8,9,12b-octahydro[1,4]dioxino[2,3-i]phenanthridine-2,3,4-triyl triacetate [(-)-53] were also demethylated selectively at the position A-7 using the abovementioned method (TMS-I, acetonitrile). As a result of this cleavage, (-)-7-hydroxy-6-oxo-1,2,3,4,4a,9,10,12b-octahydro[1,4]dioxino[2,3-j]phenanthridine-2,3,4-triyl triacetate $[(-)-54]$ was obtained in moderate yield $(55 \%)$. Then, compound (-)-52, (-)-53, or (-)-54 was also deacetylated by Zemplén's method to afford (-)-9 in good yield (76\%),

Scheme 6<smiles>[R]c1cc2c(c([R])c1[R])C(OC)=N[C@@H]1[C@@H]2C[C@H](OC(C)=O)[C@@H](OC(C)=O)[C@@H]1OC(C)=O</smiles><smiles>COCCCCO</smiles><smiles>[R]c1cc2c(c([R])c1[R])C(OC)=N[C@H]1[C@@H]2C[C@H](O)[C@@H](O)[C@@H]1O</smiles>

(-)-42 $\mathrm{R}^{1}=\mathrm{OMe}, \mathrm{R}^{2}$ and $\mathrm{R}^{3}=\mathrm{O}\left(\mathrm{CH}_{2}\right)_{2} \mathrm{O}$

$(-)-50 \mathrm{R}^{1}=\mathrm{OMe}, \mathrm{R}^{2}$ and $\mathrm{R}^{3}=\mathrm{O}\left(\mathrm{CH}_{2}\right)_{2} \mathrm{O}(22 \%)$

$(-)-43 \mathrm{R}^{1}$ and $\mathrm{R}^{2}=\mathrm{O}\left(\mathrm{CH}_{2}\right)_{2} \mathrm{O}, \mathrm{R}^{3}=\mathrm{OMe}$

$(-)-51 \mathrm{R}^{1}$ and $\mathrm{R}^{2}=\mathrm{O}\left(\mathrm{CH}_{2}\right)_{2} \mathrm{O}, \mathrm{R}^{3}=\mathrm{OMe}(23 \%)$

1. $\mathrm{HCl} / \mathrm{H}_{2} \mathrm{O}$, THF 2. $\mathrm{AcCl}$<smiles>CC(=O)O[C@H]1C[C@H]2c3cc4c(c(O)c3C(=O)N[C@H]2[C@H]1OC(C)=O)OCCO4</smiles>

$(-)-54(55 \%)$
TMS-Cl, $\mathrm{KI}$

$\mathrm{MeCN}$<smiles>[R]c1cc2c(c([R])c1[R])C(=O)N[C@@H]1[C@@H]2C[C@H](OC(C)=O)[C@@H](OC(C)=O)[C@@H]1OC(C)=O</smiles>

(-)-52 $\mathrm{R}^{1}=\mathrm{OMe}, \mathrm{R}^{2}$ and $\mathrm{R}^{3}=\mathrm{O}\left(\mathrm{CH}_{2}\right)_{2} \mathrm{O}(87 \%)$

$(-)-53 \mathrm{R}^{1}$ and $\mathrm{R}^{2}=\mathrm{O}\left(\mathrm{CH}_{2}\right)_{2} \mathrm{O}, \mathrm{R}^{3}=\mathrm{OMe}(47 \%)$

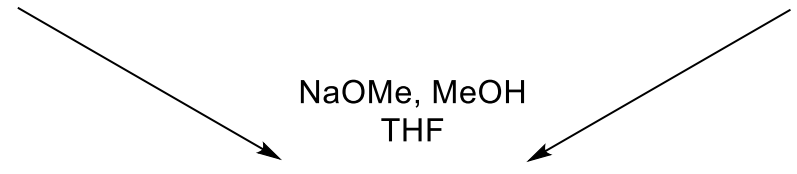

(-)-9 (76\%, ee $99 \%)$

$(-)-10(47 \%$, ee $99 \%)$

$(-)-11(47 \%$, ee $99 \%)$ 
and due to the regioisomer formation, two further synthetic analogues of trans-dihydronarciclasine (-)-10 and (-)-11 in moderate yields $(47 \%)$.

\section{Conclusion}

In conclusion, four new synthetic trans-dihydronarciclasine analogues $[(-)-8-(-)-11)]$ containing a relatively rigid $1,4-$ benzodioxane moiety in ring A were synthesised from vanillin using stereo- and enantioselective synthetic routes. These new, optically active derivatives were obtained with excellent enantiomeric purity $(99 \% e e)$. According to their negative optical rotation values, these compounds appear to be new analogues of (-)-trans-dihydronarciclasine. The preparation of compounds (-)-10 and (-)-11 was due to a side reaction and regioisomer formation, and based on our modified synthesis strategy. Biological evaluations of these potentially anticancerous and antiviral molecules are in progress.

\section{Experimental}

All reagents are commercially available from Merck. Melting points were measured on a Büchi 510 apparatus using a certified mercury thermometer (ASTM 2C). Optical rotations were measured on a Perkin Elmer 241 polarimeter. IR spectra were obtained on a PerkinElmer 1600 FT-IR instrument. NMR spectra were recorded on a Bruker AV-300 instrument. HPLC analyses were carried out with a Jasco PU-1580 apparatus equipped with a Jasco UV-1575 detector $(\lambda=256 \mathrm{~nm})$ using a Daicel Chiralpack $^{\circledR}$ OD $(250 \times 4.6 \mathrm{~mm} \times 5 \mu \mathrm{m})$ column (eluent: hexane $/ i-\mathrm{PrOH}, 8: 2$; flow rate: $2.0 \mathrm{~cm}^{3} \min ^{-1} ; 20{ }^{\circ} \mathrm{C}$ ). Elemental analyses were performed on a vario EL III instrument (Elementar Analysensysteme). Precoated silica gel plates (Merck $60 \mathrm{~F}_{254}$ ) were used for analytical TLC and Kieselgel 60 for column cromatography. Compounds 14, 15, and 22 were prepared as described previously [39], and their spectral data were found to be identical with the ones described in Ref [39].

3,4-Dihydroxybenzaldehyde (13) A solution of $35.00 \mathrm{~g} 12$ $(0.23 \mathrm{~mol})$ in $300 \mathrm{~cm}^{3}$ dichloromethane was cooled to $0{ }^{\circ} \mathrm{C}$ and subsequently $36.81 \mathrm{~g} \mathrm{AlCl}_{3}(0.28 \mathrm{~mol})$ was added. Then it was allowed to warm to $\mathrm{rt}, 81.67 \mathrm{~cm}^{3}$ pyridine $(80.20 \mathrm{~g}$, $1.01 \mathrm{~mol}$ ) was added dropwise and the reaction mixture was refluxed for 24 h. After cooling, it was acidified with $20 \%$ aqueous hydrochloric acid to $\mathrm{pH}=2$. The precipitated pyridinium salt was dissolved by adding $300 \mathrm{~cm}^{3}$ water and then the aqueous phase was extracted with ethyl acetate $\left(4 \times 250 \mathrm{~cm}^{3}\right)$. The combined organic layer was washed with brine, dried over $\mathrm{Na}_{2} \mathrm{SO}_{4}$, and evaporated in vacuo to give $29.22 \mathrm{~g} 13$ (92\%) as a pale yellow solid, which was used without further purification. M.p.: $152-153{ }^{\circ} \mathrm{C}$ (Ref. [41] $153-154^{\circ} \mathrm{C}$ ); its spectral data were found to be identical with the ones described in Ref. [62].

\section{General procedure for the synthesis of 1,4- benzodioxanes 16 and 17}

$43.80 \mathrm{~g}$ potassium carbonate $(0.32 \mathrm{~mol})$ and $15.1 \mathrm{~cm}^{3} 1,2-$ dibromoethane $(32.90 \mathrm{~g}, 0.18 \mathrm{~mol})$ were added to a solution of $22.00 \mathrm{~g} 13(0.16 \mathrm{~mol})$ or $26.70 \mathrm{~g} 15(0.16 \mathrm{~mol})$ in $250 \mathrm{~cm}^{3}$ DMF. The reaction mixture was stirred at 100-110 ${ }^{\circ} \mathrm{C}$ for $4-8 \mathrm{~h}$. After cooling to $\mathrm{rt}$, the precipitated inorganic salts were filtered and the reaction mixture was evaporated to $80 \mathrm{~cm}^{3}$ in vacuo. The residue was poured into $670 \mathrm{~cm}^{3}$ water. The product was isolated as specified.

2,3-Dihydrobenzo[b][1,4]dioxine-6-carbaldehyde (16) The aqueous phase was extracted with ethyl acetate $\left(4 \times 200 \mathrm{~cm}^{3}\right)$, and the combined organic layer was washed with brine and dried over $\mathrm{Na}_{2} \mathrm{SO}_{4}$. The crude product was purified by distillation in vacuo to afford $\mathbf{1 6}$. Yield: 63\%; white solid; m.p.: 48-49 ${ }^{\circ} \mathrm{C}$ (Ref. [63] 49.5$\left.50.5^{\circ} \mathrm{C}\right)$; b.p.: $105-108{ }^{\circ} \mathrm{C} \quad(0.3 \mathrm{mbar}) ;{ }^{1} \mathrm{H} \quad \mathrm{NMR}$ (300 $\left.\mathrm{MHz}, \mathrm{CDCl}_{3}\right): \delta=9.82(\mathrm{~s}, 1 \mathrm{H}, \mathrm{CHO}), 7.42-7.38(\mathrm{~m}$, $2 \mathrm{H}, 5-\mathrm{H}_{\mathrm{Ar}}$ and $\left.7-\mathrm{H}_{\mathrm{Ar}}\right), 6.98\left(\mathrm{~d}, J=8.7 \mathrm{~Hz}, 1 \mathrm{H}, 8-\mathrm{H}_{\mathrm{Ar}}\right)$, 4.35-4.28 (m, 4H, $\left.\mathrm{OCH}_{2} \mathrm{CH}_{2} \mathrm{O}\right) \mathrm{ppm} ;{ }^{13} \mathrm{C} \mathrm{NMR}(75 \mathrm{MHz}$, $\left.\mathrm{CDCl}_{3}\right): \delta=64.0\left(\mathrm{OCH}_{2} \mathrm{CH}_{2} \mathrm{O}\right), 64.7\left(\mathrm{OCH}_{2} \mathrm{CH}_{2} \mathrm{O}\right), 117.8$ $\left(5-\mathrm{C}_{\mathrm{Ar}}\right.$ or $\left.8-\mathrm{C}_{\mathrm{Ar}}\right), 118.4\left(5-\mathrm{C}_{\mathrm{Ar}}\right.$ or $\left.8-\mathrm{C}_{\mathrm{Ar}}\right), 124.2\left(7-\mathrm{C}_{\mathrm{Ar}}\right)$, $130.7\left(6-\mathrm{C}_{\mathrm{Ar}}\right), 143.9 \quad\left(4 \mathrm{a}-\mathrm{C}_{\mathrm{Ar}}\right), 149.1 \quad\left(8 \mathrm{a}-\mathrm{C}_{\mathrm{Ar}}\right), 190.7$ (CHO) ppm; IR (KBr): $\bar{v}=3001,2883,1687,1581,1506$, 1458, 1394, 1291, 1156, 1062, 887, $777 \mathrm{~cm}^{-1}$.

\section{8-Methoxy-2,3-dihydrobenzo[b][1,4]dioxine-6-carbalde-}

hyde (17) The precipitated crystals were filtered, washed with water, and dried to give 17, which was used without further purification. Yield: 63\%; white crystals; m.p.: 78$79{ }^{\circ} \mathrm{C}$. (Ref. [64] 69-72 ${ }^{\circ} \mathrm{C}$ ); ${ }^{1} \mathrm{H}$ NMR (300 MHz, $\mathrm{CDCl}_{3}$ ): $\delta=9.77(\mathrm{~s}, 1 \mathrm{H}, \mathrm{CHO}), 7.06\left(\mathrm{~s}, 2 \mathrm{H}, 5-\mathrm{H}_{\mathrm{Ar}}\right.$ and $\left.7-\mathrm{H}_{\mathrm{Ar}}\right)$, 4.40-4.28 (m, 4H, $\left.\mathrm{OCH}_{2} \mathrm{CH}_{2} \mathrm{O}\right), 3.93$ (s, 3H, $\left.\mathrm{OCH}_{3}\right) \mathrm{ppm}$; ${ }^{13} \mathrm{C} \mathrm{NMR}\left(75 \mathrm{MHz}, \mathrm{CDCl}_{3}\right): \delta=56.3\left(\mathrm{OCH}_{3}\right), 63.9$ $\left(\mathrm{OCH}_{2} \mathrm{CH}_{2} \mathrm{O}\right), 64.9\left(\mathrm{OCH}_{2} \mathrm{CH}_{2} \mathrm{O}\right), 103.0\left(7-\mathrm{C}_{\mathrm{Ar}}\right), 114.5$ (5$\left.\mathrm{C}_{\mathrm{Ar}}\right), 129.2\left(6-\mathrm{C}_{\mathrm{Ar}}\right), 138.8\left(8 \mathrm{a}-\mathrm{C}_{\mathrm{Ar}}\right), 144.0\left(8-\mathrm{C}_{\mathrm{Ar}}\right), 149.6$ $\left(4 \mathrm{a}-\mathrm{C}_{\mathrm{Ar}}\right), 190.7$ (CHO) ppm; IR $(\mathrm{KBr}): \bar{v}=2982,2948$, 2892, 1698, 1590, 1503, 1470, 1454, 1392, 1324, 1125, $1046,891,840 \mathrm{~cm}^{-1}$.

\section{General procedure for the synthesis of 2,3- dihydrobenzodioxine butenones 18 and 19}

A solution of $16.46 \mathrm{~g} \mathbf{1 6}(0.10 \mathrm{~mol})$ or $19.41 \mathrm{~g} \quad \mathbf{1 7}$ $(0.10 \mathrm{~mol})$ in $95 \mathrm{~cm}^{3}$ acetone $(75.15 \mathrm{~g}, 1.29 \mathrm{~mol})$ was added into $42 \mathrm{~cm}^{3}$ water; then the starting material was precipitated in a fine crystal form. Aqueous sodium 
hydroxide solution (from $1.51 \mathrm{~g}(37.76 \mathrm{mmol}) \mathrm{NaOH}$ and $6.8 \mathrm{~cm}^{3} \mathrm{H}_{2} \mathrm{O}$ ) and $378 \mathrm{~cm}^{3}$ water were also added and the yellow mixture was stirred for $20 \mathrm{~h}$ intensively at room temperature. The yellow crude product was filtered, washed with water, and dried. Finally, it was purified by distillation in vacuo to afford $\mathbf{1 8}$ or $\mathbf{1 9}$.

4-(2,3-Dihydrobenzo[b][1,4]dioxin-6-yl)but-3-en-2-one (18, $\left.\mathrm{C}_{12} \mathrm{H}_{12} \mathrm{O}_{3}\right)$ Yield: 83\%; white crystals; m.p.: 90-91 ${ }^{\circ} \mathrm{C}$; b.p.: $153{ }^{\circ} \mathrm{C}$ (0.2 mbar); ${ }^{1} \mathrm{H}$ NMR (300 MHz, $\left.\mathrm{CDCl}_{3}\right)$ : $\delta=7.41(\mathrm{~d}, J=16.2 \mathrm{~Hz}, 1 \mathrm{H}, \mathrm{Ar}-\mathrm{CH}=\mathrm{CH}), 7.08-7.04$ $\left(\mathrm{m}, 2 \mathrm{H}, 5-\mathrm{H}_{\mathrm{Ar}}\right.$ and $\left.7-\mathrm{H}_{\mathrm{Ar}}\right), 6.88\left(\mathrm{~d}, J=8.4 \mathrm{~Hz}, 1 \mathrm{H}, 8-\mathrm{H}_{\mathrm{Ar}}\right)$, $6.58(\mathrm{~d}, J=16.2 \mathrm{~Hz}, 1 \mathrm{H}, \mathrm{Ar}-\mathrm{CH}=\mathrm{CH}), 4.31-4.27(\mathrm{~m}$, $\left.4 \mathrm{H}, \mathrm{OCH}_{2} \mathrm{CH}_{2} \mathrm{O}\right), 2.36$ (s, 3H, $\left.\mathrm{COCH}_{3}\right) \mathrm{ppm} ;{ }^{13} \mathrm{C} \mathrm{NMR}$ $\left(75 \mathrm{MHz}, \mathrm{CDCl}_{3}\right): \delta=27.4\left(\mathrm{COCH}_{3}\right), 64.2\left(\mathrm{OCH}_{2} \mathrm{CH}_{2} \mathrm{O}\right)$, $64.5\left(\mathrm{OCH}_{2} \mathrm{CH}_{2} \mathrm{O}\right), 116.8\left(5-\mathrm{C}_{\mathrm{Ar}}\right.$ or $\left.8-\mathrm{C}_{\mathrm{Ar}}\right), 117.8\left(5-\mathrm{C}_{\mathrm{Ar}}\right.$ or $\left.8-\mathrm{C}_{\mathrm{Ar}}\right), 122.3\left(7-\mathrm{C}_{\mathrm{Ar}}\right), 125.5(\mathrm{Ar}-\mathrm{CH}=\mathrm{CH}), 128.0(6-$ $\left.\mathrm{C}_{\mathrm{Ar}}\right), 143.1(\mathrm{Ar}-\mathrm{CH}=\mathrm{CH}), 143.7\left(4 \mathrm{a}-\mathrm{C}_{\mathrm{Ar}}\right), 149.2(8 \mathrm{a}-$ $\mathrm{C}_{\mathrm{Ar}}$ ), 192.2 (CO) ppm; IR (KBr): $\bar{v}=2989,2894,1668$, 1641, 1579, 1513, 1455, 1427, 1360, 1300, 1251, 1122, $1061,977,884,806,779 \mathrm{~cm}^{-1}$.

4-(8-Methoxy-2,3-dihydrobenzo[b][1,4]dioxin-6-yl)but-3-en2-one (19, $\left.\mathrm{C}_{13} \mathrm{H}_{14} \mathrm{O}_{4}\right)$ Yield: $62 \%$; light yellow solid; m.p.: 96-98 ${ }^{\circ} \mathrm{C}$; b.p.: $183{ }^{\circ} \mathrm{C}(0.2 \mathrm{mbar}) ;{ }^{1} \mathrm{H}$ NMR $(300 \mathrm{MHz}$, $\left.\mathrm{CDCl}_{3}\right): \delta=7.37(\mathrm{~d}, J=16.2 \mathrm{~Hz}, \mathrm{Ar}-\mathrm{CH}=\mathrm{CH}), 6.75(\mathrm{~d}$, $J=1.8 \mathrm{~Hz}, 1 \mathrm{H}, 5-\mathrm{H}_{\mathrm{Ar}}$ or $\left.7-\mathrm{H}_{\mathrm{Ar}}\right), 6.69(\mathrm{~d}, J=1.8 \mathrm{~Hz}, 1 \mathrm{H}$, $5-\mathrm{H}_{\mathrm{Ar}}$ or $\left.7-\mathrm{H}_{\mathrm{Ar}}\right), 6.57(\mathrm{~d}, J=15.9 \mathrm{~Hz}, 1 \mathrm{H}, \mathrm{Ar}-\mathrm{CH}=\mathrm{CH})$, 4.36-4.26 (m, 4H, $\left.\mathrm{OCH}_{2} \mathrm{CH}_{2} \mathrm{O}\right), 3.90\left(\mathrm{~s}, 3 \mathrm{H}, \mathrm{OCH}_{3}\right), 2.35$ (s, 3H, $\left.\mathrm{COCH}_{3}\right) \mathrm{ppm} ;{ }^{13} \mathrm{C} \mathrm{NMR}\left(75 \mathrm{MHz}, \mathrm{CDCl}_{3}\right)$ : $\delta=27.4\left(\mathrm{COCH}_{3}\right), 56.2\left(\mathrm{OCH}_{3}\right), 64.1\left(\mathrm{OCH}_{2} \mathrm{CH}_{2} \mathrm{O}\right), 64.7$ $\left(\mathrm{OCH}_{2} \mathrm{CH}_{2} \mathrm{O}\right), 103.6\left(7-\mathrm{C}_{\mathrm{Ar}}\right), 111.0\left(5-\mathrm{C}_{\mathrm{Ar}}\right), 125.8(\mathrm{Ar}-$ $\mathrm{CH}=\mathrm{CH}), 126.8\left(6-\mathrm{C}_{\mathrm{Ar}}\right), 135.5\left(8 \mathrm{a}-\mathrm{C}_{\mathrm{Ar}}\right), 143.4(\mathrm{Ar}-$ $\mathrm{CH}=\mathrm{CH}), 144.1\left(8-\mathrm{C}_{\mathrm{Ar}}\right), 149.2\left(4 \mathrm{a}-\mathrm{C}_{\mathrm{Ar}}\right), 192.2(\mathrm{CO})$ ppm; IR (KBr): $\bar{v}=2996,2940,1666,1638,1589,1508$, $1453,1321,1274,1126,977,822 \mathrm{~cm}^{-1}$.

\section{General procedure for the synthesis of racemic nitropentanones $( \pm)-20$ and $( \pm)$-21}

To a solution of $7.70 \mathrm{~g} \mathbf{1 8}$ (37.74 mmol) or $8.84 \mathrm{~g} \mathbf{1 9}$ (37.74 mmol) in a mixture of anhydrous $15.1 \mathrm{~cm}^{3}$ ethanol and $10.22 \mathrm{~cm}^{3}$ nitromethane $(11.52 \mathrm{~g}, 0.19 \mathrm{~mol})$ was added $0.11 \mathrm{~g}$ anhydrous potassium carbonate $(0.81 \mathrm{mmol})$ and the reaction mixture was refluxed for 5-8 h. Then, it was cooled to $\mathrm{rt}$ and $14.3 \mathrm{~cm}^{3}$ water was added. The product was isolated as specified.

\section{( \pm )-4-(2,3-Dihydrobenzo[b][1,4]dioxin-6-yl)-5-nitropentan-}

2-one $\left[( \pm)-20, \mathrm{C}_{13} \mathrm{H}_{15} \mathrm{NO}_{5}\right]$ The aqueous phase was extracted with ethyl acetate $\left(3 \times 40 \mathrm{~cm}^{3}\right)$, and the combined organic layer was washed with brine, dried over $\mathrm{Na}_{2} \mathrm{SO}_{4}$, and evaporated in vacuo. Recrystallisation from ethyl acetate gave (土)-20. Yield: 75\%; white crystals; m.p.: $101-102{ }^{\circ} \mathrm{C} ; \quad R_{f}=0.52$ (hexane/EtOAc, $1: 1$ ); ${ }^{1} \mathrm{H}$ NMR $\left(300 \mathrm{MHz} \mathrm{CDCl}_{3}\right): \delta=6.80(\mathrm{~d}, J=8.1 \mathrm{~Hz}, 1 \mathrm{H}, 8-$ $\left.\mathrm{H}_{\mathrm{Ar}}\right), 6.70\left(\mathrm{~d}, J=1.8 \mathrm{~Hz}, 1 \mathrm{H}, 5-\mathrm{H}_{\mathrm{Ar}}\right), 6.67(\mathrm{dd}, J=8.4$, $\left.2.1 \mathrm{~Hz}, 1 \mathrm{H}, 7-\mathrm{H}_{\mathrm{Ar}}\right), 4.63\left(\mathrm{dd}, J=12.3,6.9 \mathrm{~Hz}, 1 \mathrm{H}, \mathrm{CH}_{2-}\right.$ $\mathrm{NO}_{2}$ ), $4.53\left(\mathrm{dd}, J=12.3,7.8 \mathrm{~Hz}, 1 \mathrm{H}, \mathrm{CH}_{2} \mathrm{NO}_{2}\right), 4.23(\mathrm{~s}$, $4 \mathrm{H}, \mathrm{OCH}_{2} \mathrm{CH}_{2} \mathrm{O}$ ), 3.89 (quint, $J=7.2 \mathrm{~Hz}, 1 \mathrm{H}, \mathrm{Ar}-\mathrm{CH}$ ), $2.86\left(\mathrm{~d}, J=7.2 \mathrm{~Hz}, 2 \mathrm{H}, \mathrm{CH}_{2} \mathrm{CO}\right), 2.12\left(\mathrm{~s}, 3 \mathrm{H}, \mathrm{COCH}_{3}\right)$ ppm; ${ }^{13} \mathrm{C} \mathrm{NMR}\left(75 \mathrm{MHz}, \mathrm{CDCl}_{3}\right): \delta=30.4\left(\mathrm{COCH}_{3}\right)$, $38.4(\mathrm{Ar}-\mathrm{CH}), 46.2\left(\mathrm{CH}_{2} \mathrm{CO}\right), 64.3\left(\mathrm{OCH}_{2} \mathrm{CH}_{2} \mathrm{O}\right), 64.3$ $\left(\mathrm{OCH}_{2} \mathrm{CH}_{2} \mathrm{O}\right), 79.6\left(\mathrm{CH}_{2} \mathrm{NO}_{2}\right), 116.1\left(5-\mathrm{C}_{\mathrm{Ar}}\right.$ or $\left.8-\mathrm{C}_{\mathrm{Ar}}\right)$, $117.7\left(5-\mathrm{C}_{\mathrm{Ar}}\right.$ or $\left.8-\mathrm{C}_{\mathrm{Ar}}\right), 120.3\left(7-\mathrm{C}_{\mathrm{Ar}}\right), 131.9\left(6-\mathrm{C}_{\mathrm{Ar}}\right), 143.1$ $\left(8 \mathrm{a}-\mathrm{C}_{\mathrm{Ar}}\right.$ or $\left.4 \mathrm{a}-\mathrm{C}_{\mathrm{Ar}}\right), 143.7\left(4 \mathrm{a}-\mathrm{C}_{\mathrm{Ar}}\right.$ or $\left.8 \mathrm{a}-\mathrm{C}_{\mathrm{Ar}}\right), 205.4(\mathrm{CO})$ ppm; IR (KBr): $\bar{v}=2878,1717,1592,1508,1433,1384$, $1313,1220,1161,1130,1071,903,819,639 \mathrm{~cm}^{-1}$.

$( \pm)-4-(8-M e t h o x y-2,3-d i h y d r o b e n z o[b][1,4]$ dioxin-6-yl)-5-nitropentan-2-one [( \pm )-21, $\left.\mathrm{C}_{14} \mathrm{H}_{17} \mathrm{NO}_{6}\right]$ After crystallisation at $0{ }^{\circ} \mathrm{C}$, the precipitated crystals were filtered, washed with water and dried to afford ( \pm )-21. Yield: $86 \%$; white solid; m.p.: 99-101 ${ }^{\circ} \mathrm{C} ; R_{f}=0.33$ (hexane/EtOAc, $1: 1$ ); ${ }^{1} \mathrm{H}$ NMR $\left(300 \mathrm{MHz} \mathrm{CDCl}_{3}\right): \delta=6.35\left(\mathrm{~d}, J=1.8 \mathrm{~Hz}, 1 \mathrm{H}, 5-\mathrm{H}_{\mathrm{Ar}}\right.$ or $\left.7-\mathrm{H}_{\mathrm{Ar}}\right), 6.34\left(\mathrm{~d}, J=1.8 \mathrm{~Hz}, 1 \mathrm{H}, 5-\mathrm{H}_{\mathrm{Ar}}\right.$ or $\left.7-\mathrm{H}_{\mathrm{Ar}}\right), 4.63(\mathrm{dd}$, $\left.J=12.3,6.9 \mathrm{~Hz}, 1 \mathrm{H}, \mathrm{CH}_{2} \mathrm{NO}_{2}\right), 4.53(\mathrm{dd}, J=12.3$, $\left.7.8 \mathrm{~Hz}, 1 \mathrm{H}, \mathrm{CH}_{2} \mathrm{NO}_{2}\right), 4.29-4.22\left(\mathrm{~m}, 4 \mathrm{H}, \mathrm{OCH}_{2} \mathrm{CH}_{2} \mathrm{O}\right)$, 3.88 (quint, $J=7.2 \mathrm{~Hz}, 1 \mathrm{H}, \mathrm{Ar}-\mathrm{CH}), 3.86\left(\mathrm{~s}, 3 \mathrm{H}, \mathrm{OCH}_{3}\right)$, $2.86\left(\mathrm{dd}, J=6.9,1.5 \mathrm{~Hz}, 2 \mathrm{H}, \mathrm{CH}_{2} \mathrm{CO}\right), 2.12(\mathrm{~s}, 3 \mathrm{H}$, $\mathrm{COCH}_{3}$ ) ppm; ${ }^{13} \mathrm{C}$ NMR $\left(75 \mathrm{MHz}, \mathrm{CDCl}_{3}\right): \delta=30.4$ $\left(\mathrm{COCH}_{3}\right), 38.9(\mathrm{Ar}-\mathrm{CH}), 46.2\left(\mathrm{CH}_{2} \mathrm{CO}\right), 56.2\left(\mathrm{OCH}_{3}\right)$, $64.2\left(\mathrm{OCH}_{2} \mathrm{CH}_{2} \mathrm{O}\right), 64.4\left(\mathrm{OCH}_{2} \mathrm{CH}_{2} \mathrm{O}\right), 79.5\left(\mathrm{CH}_{2} \mathrm{NO}_{2}\right)$, $103.8\left(7-\mathrm{C}_{\mathrm{Ar}}\right), 108.3\left(5-\mathrm{C}_{\mathrm{Ar}}\right), 131.0\left(8 \mathrm{a}-\mathrm{C}_{\mathrm{Ar}}\right), 132.6(6-$ $\left.\mathrm{C}_{\mathrm{Ar}}\right), 144.2\left(8-\mathrm{C}_{\mathrm{Ar}}\right), 149.4\left(4 \mathrm{a}-\mathrm{C}_{\mathrm{Ar}}\right), 205.4$ (CO) ppm; IR $(\mathrm{KBr}): \bar{v}=2962,1716,1599,1513,1438,1386,1326$, $1221,1130,1046,951,844,669 \mathrm{~cm}^{-1}$.

\section{General procedure for the enantioselective Michael addition using organocatalyst 22}

A solution of $8.17 \mathrm{~g} \mathbf{1 8}(40.00 \mathrm{mmol})$ or $9.37 \mathrm{~g} \mathbf{1 9}$ (40.00 mmol) and $20 \mathrm{~mol} \% \quad(8 S, 9 S)$-9-amino(9-deoxy)epiquinine (22) in $54 \mathrm{~cm}^{3}$ anhydrous nitromethane was stirred at rt for $7 \mathrm{~d}$, and then the solvent was evaporated in vacuo. The residue was purified by column chromatography (hexane/EtOAc, 1:1) to obtain optically active (-)-20 or recrystallised from $\mathrm{MeOH}$ to give (-)-21. Spectroscopic data and elemental analysis for these compounds matched those for the racemates as given above.

(-)-4-(2,3-Dihydrobenzo[b][1,4]dioxin-6-yl)-5-nitropentan2-one [(-)-20, $\left.\mathrm{C}_{13} \mathrm{H}_{15} \mathrm{NO}_{5}\right]$ Yield: $67 \%$; white solid; m.p.: 75-77 ${ }^{\circ} \mathrm{C} ; R_{f}=0.52$ (hexane/EtOAc, 1:1); HPLC: Chiralpack $^{\circledR}$ OD (hexane $/ i-\mathrm{PrOH}=8: 2$, flow rate $2.0 \mathrm{~cm}^{3} \mathrm{~min}^{-1}$, $\left.256 \mathrm{~nm}, 20{ }^{\circ} \mathrm{C}\right), t_{(-)}: 25 \mathrm{~min}, t_{(+)}: 18 \mathrm{~min} ;[\alpha]_{\mathrm{D}}^{22}=-16.1^{\circ}$ ( $c=0.94$, acetone); ee $92 \%$. 
(-)-4-(8-Methoxy-2,3-dihydrobenzo[b][1,4]dioxin-6-yl)-5nitropentan-2-one [(-)-21, $\left.\mathrm{C}_{14} \mathrm{H}_{17} \mathrm{NO}_{6}\right]$ Yield: $67 \%$; white crystals; m.p.: $111-115{ }^{\circ} \mathrm{C} ; R_{f}=0.33$ (hexane/EtOAc, 1:1); HPLC: Chiralpack ${ }^{\circledR}$ OD (hexane $/ i-\mathrm{PrOH}=8: 2$, flow rate $\left.2.0 \mathrm{~cm}^{3} \mathrm{~min}^{-1}, 256 \mathrm{~nm}, 20{ }^{\circ} \mathrm{C}\right), t_{(-)}: 32 \mathrm{~min}, t_{(+)}$: $28 \min ;[\alpha]_{\mathrm{D}}^{22}=-15.0^{\circ}(c=1.5$, acetone $)$; ee $98 \%$.

\section{General procedure for the Claisen-Henry reaction}

Dry and freshly prepared sodium methoxide powder (from $2.90 \mathrm{~g}$ sodium $(0.13 \mathrm{~mol})$ and $70 \mathrm{~cm}^{3}$ anhydrous methanol) was suspended in $100 \mathrm{~cm}^{3}$ anhydrous diethyl ether. Subsequently, $14.50 \mathrm{~cm}^{3}$ ethyl formate $(13.36 \mathrm{~g}, 0.18 \mathrm{~mol})$ and $8.07 \mathrm{~g} \quad(-)-20 \quad(30.48 \mathrm{mmol})$ or $9.00 \mathrm{~g}(-)-21$ $(30.48 \mathrm{mmol})$ were added, and the reaction mixture was stirred at $\mathrm{rt}$ for $20 \mathrm{~h}$. It was cooled to $0{ }^{\circ} \mathrm{C}$ and $51 \mathrm{~cm}^{3}$ water was added dropwise. After separating, the aqueous phase was acidified with acetic acid to $\mathrm{pH}=4$ at $0{ }^{\circ} \mathrm{C}$. The precipitated crystals were filtered, washed with water and dried. The crude product (-)-23 or (-)-24 was purified as specified.

(-)-3-Hydroxy-5-(2',3'-dihydrobenzo[b][1,4]dioxin- $6^{\prime}$-yl)4-nitrocyclohexanone [(-)-23, $\left.\mathrm{C}_{14} \mathrm{H}_{15} \mathrm{NO}_{6}\right]$ Recrystallisation from ethyl acetate gave light yellow crystals. Yield: 46\%; m.p.: $206{ }^{\circ} \mathrm{C}$; ${ }^{1} \mathrm{H}$ NMR (300 MHz, DMSO- $d_{6}$ ): $\delta=6.96\left(\mathrm{~d}, J=1.8 \mathrm{~Hz}, 1 \mathrm{H}, 5-\mathrm{H}_{\mathrm{Ar}}\right), 6.86(\mathrm{dd}, J=8.4$, $\left.1.8 \mathrm{~Hz}, 1 \mathrm{H}, 7-\mathrm{H}_{\mathrm{Ar}}\right), 6.78\left(\mathrm{~d}, J=8.1 \mathrm{~Hz}, 1 \mathrm{H}, 8-\mathrm{H}_{\mathrm{Ar}}\right), 5.98$ (d, $J=4.5 \mathrm{~Hz}, 1 \mathrm{H}, \mathrm{OH}), 5.67$ (dd, $J=11.7,2.1 \mathrm{~Hz}, 1 \mathrm{H}, 4-$ $\mathrm{H})$, 4.69-4.63 (m, 1H, 3- $\left.\mathrm{H}_{\mathrm{Cy}}\right), 4.21\left(\mathrm{~s}, 4 \mathrm{H}, \mathrm{OCH}_{2} \mathrm{CH}_{2} \mathrm{O}\right)$, $3.83(\mathrm{td}, J=12.9,4.5 \mathrm{~Hz}, 1 \mathrm{H}, 5-\mathrm{H}), 3.04(\mathrm{dd}, J=14.4$, $\left.2.7 \mathrm{~Hz}, 1 \mathrm{H}, 2-\mathrm{H}_{\beta, C y}\right), 2.65$ (t, $\left.J=14.1 \mathrm{~Hz}, 1 \mathrm{H}, 6-\mathrm{H}_{\beta}\right), 2.39$ (dt, $\left.J=14.4,3.0 \mathrm{~Hz}, 1 \mathrm{H}, 2-\mathrm{H}_{\alpha, \mathrm{Cy}}\right), 2.31$ (ddd, $J=14.7$, 5.1, $2.1 \mathrm{~Hz}, 1 \mathrm{H}, 6-\mathrm{H}_{\alpha}$ ) ppm; ${ }^{13} \mathrm{C}$ NMR $(75 \mathrm{MHz}$, DMSO$\left.d_{6}\right): \quad \delta=40.4 \quad(5-\mathrm{C}), 46.4 \quad\left(2-\mathrm{C}_{\mathrm{Cy}}\right), 47.3 \quad(6-\mathrm{C}), 63.9$ $\left(\mathrm{OCH}_{2} \mathrm{CH}_{2} \mathrm{O}\right), 64.0\left(\mathrm{OCH}_{2} \mathrm{CH}_{2} \mathrm{O}\right), 69.6\left(3-\mathrm{C}_{\mathrm{Cy}}\right), 89.4$ (4C), $115.7\left(8-\mathrm{C}_{\mathrm{Ar}}\right), 117.0\left(5-\mathrm{C}_{\mathrm{Ar}}\right), 120.1\left(7-\mathrm{C}_{\mathrm{Ar}}\right), 133.6$ (6$\left.\mathrm{C}_{\mathrm{Ar}}\right), 142.3\left(8 \mathrm{a}-\mathrm{C}_{\mathrm{Ar}}\right), 143.2\left(4 \mathrm{a}-\mathrm{C}_{\mathrm{Ar}}\right), 206.0$ (CO) ppm; IR $(\mathrm{KBr}): \bar{v}=3318,2984,2919,1714,1591,1554,1511$, 1461, 1373, 1309, 1248, 1127, 1067, 889, $814 \mathrm{~cm}^{-1}$; $[\alpha]_{\mathrm{D}}^{22}=-18.4^{\circ}\left(c=1, \mathrm{CHCl}_{3}\right)$.

(-)-3-Hydroxy-5-(8'-methoxy-2', 3'-dihydrobenzo[b][1,4]dioxin-6'-yl)-4-nitrocyclohexanone $\left[(-)-24, \mathrm{C}_{15} \mathrm{H}_{17} \mathrm{NO}_{7}\right]$ It was purified by column chromatography (hexane/EtOAc, 1:2) to afford a light yellow solid. Yield: 57\%; m.p.: 200$201{ }^{\circ} \mathrm{C} ; \quad R_{f}=0.19 \quad$ (hexane/EtOAc, $1: 1$ ); ${ }^{1} \mathrm{H} \quad \mathrm{NMR}$ (300 MHz, DMSO- $d_{6}$ ): $\delta=6.66(\mathrm{~d}, J=1.2 \mathrm{~Hz}, 1 \mathrm{H}, 5-$ $\left.\mathrm{H}_{\mathrm{Ar}}\right), 6.56\left(\mathrm{~d}, J=1.5 \mathrm{~Hz}, 1 \mathrm{H}, 7-\mathrm{H}_{\mathrm{Ar}}\right), 5.99(\mathrm{~d}, J=4.5 \mathrm{~Hz}$, $1 \mathrm{H}, \mathrm{OH}), 5.70(\mathrm{dd}, J=11.7,1.8 \mathrm{~Hz}, 1 \mathrm{H}, 4-\mathrm{H}), 4.70-4.64$ $\left(\mathrm{m}, 1 \mathrm{H}, 3-\mathrm{H}_{\mathrm{Cy}}\right), 4.18\left(\mathrm{~s}, 4 \mathrm{H}, \mathrm{OCH}_{2} \mathrm{CH}_{2} \mathrm{O}\right), 3.81$ (td, $J=12.9,4.2 \mathrm{~Hz}, 1 \mathrm{H}, 5-\mathrm{H}), 3.74\left(\mathrm{~s}, 3 \mathrm{H}, \mathrm{OCH}_{3}\right), 3.02$ (dd, $\left.J=14.1,2.1 \mathrm{~Hz}, 1 \mathrm{H}, 2-\mathrm{H}_{\beta, \mathrm{Cy}}\right), 2.67$ (t, $J=14.1 \mathrm{~Hz}, 1 \mathrm{H}, 6-$
$\mathrm{H}_{\beta}$ ), 2.40 (dt, $J=14.7,2.7 \mathrm{~Hz}, 1 \mathrm{H}, 2-\mathrm{H}_{\alpha, \mathrm{Cy}}$ ), 2.33 (ddd, $\left.J=14.7,4.5,2.1 \mathrm{~Hz}, 1 \mathrm{H}, 6-\mathrm{H}_{\alpha}\right) \mathrm{ppm} ;{ }^{13} \mathrm{C} \mathrm{NMR}(75 \mathrm{MHz}$, DMSO- $\left.d_{6}\right): \delta=39.3(5-\mathrm{C}), 46.4\left(2-\mathrm{C}_{\mathrm{Cy}}\right), 47.3(6-\mathrm{C}), 55.8$ $\left(\mathrm{OCH}_{3}\right), 63.6\left(\mathrm{OCH}_{2} \mathrm{CH}_{2} \mathrm{O}\right), 63.9\left(\mathrm{OCH}_{2} \mathrm{CH}_{2} \mathrm{O}\right), 69.6$ $\left.\mathrm{C}_{\mathrm{Cy}}\right), 89.2$ (4-C), $104.0\left(7-\mathrm{C}_{\mathrm{Ar}}\right), 108.1\left(5-\mathrm{C}_{\mathrm{Ar}}\right), 132.0(8 \mathrm{a}-$ $\left.\mathrm{C}_{\mathrm{Ar}}\right), 132.6\left(6-\mathrm{C}_{\mathrm{Ar}}\right), 143.5\left(4 \mathrm{a}-\mathrm{C}_{\mathrm{Ar}}\right), 148.6\left(8-\mathrm{C}_{\mathrm{Ar}}\right), 206.1$ (CO) ppm; IR (KBr): $\bar{v}=3337,2962,2867,1709,1599$, 1554, 1514, 1465, 1376, 1253, 1130, 1050, 888, $825 \mathrm{~cm}^{-1}$; $[\alpha]_{\mathrm{D}}^{22}=-11.4^{\circ}(c=0.5, \mathrm{THF})$.

\section{General procedure for the synthesis of ethylene acetals (+)-25 and (+)-26}

$36 \mathrm{~cm}^{3}$ ethylene glycol $(39.89 \mathrm{~g}, 0.64 \mathrm{~mol})$ and $4.40 \mathrm{~g}$ (-)-23 (15.00 mmol) or $4.85 \mathrm{~g} \mathrm{(-)-24} \mathrm{(15.00} \mathrm{mmol)} \mathrm{were}$ added to a solution of $12.62 \mathrm{~g}$ anhydrous oxalic acid $(0.14 \mathrm{~mol})$ in $213 \mathrm{~cm}^{3}$ anhydrous acetonitrile. The mixture was stirred at $\mathrm{rt}$ for 3-4 d. Then, it was poured into a cooled and saturated $594 \mathrm{~cm}^{3} \mathrm{NaHCO}_{3}$ solution. The precipitated solid was collected by filtration, washed with water, and dried to give $(+)-\mathbf{2 5}$ or $(+)-\mathbf{2 6}$.

(+)-3-Hydroxy-5-(2',3'-dihydrobenzo[b][1,4]dioxin-6'-yl)4-nitrocyclohexanone ethylene acetal [(+)-25, $\mathrm{C}_{16} \mathrm{H}_{19}$ $\left.\mathrm{NO}_{7}\right]$ Yield: 84\%; white solid; m.p.: $224-228{ }^{\circ} \mathrm{C} ;{ }^{1} \mathrm{H}$ NMR $\left(300 \mathrm{MHz}, \mathrm{CDCl}_{3}\right): \delta=6.79(\mathrm{~d}, J=8.1 \mathrm{~Hz}, 1 \mathrm{H}, 8-$ $\left.\mathrm{H}_{\mathrm{Ar}}\right), 6.75-6.70\left(\mathrm{~m}, 2 \mathrm{H}, 5-\mathrm{H}_{\mathrm{Ar}}\right.$ and $\left.7-\mathrm{H}_{\mathrm{Ar}}\right), 4.70(\mathrm{dd}$, $J=12.0,3.0 \mathrm{~Hz}, 1 \mathrm{H}, 4-\mathrm{H}), 4.63(\mathrm{dq}, J=10.2,3.0 \mathrm{~Hz}, 1 \mathrm{H}$, 3- $\left.\mathrm{H}_{\mathrm{Cy}}\right), 4.22$ (s, 4H, $\left.\mathrm{OCH}_{2} \mathrm{CH}_{2} \mathrm{O}_{\text {benzodioxane }}\right), 4.09-3.91(\mathrm{~m}$, $5 \mathrm{H}, \mathrm{OH}$ and $\left.\mathrm{OCH}_{2} \mathrm{CH}_{2} \mathrm{O}_{\text {acetal }}\right), 3.75(\mathrm{td}, J=12.6,3.9 \mathrm{~Hz}$, $1 \mathrm{H}, 5-\mathrm{H}), 2.19\left(\mathrm{dt}, J=14.4,3.0 \mathrm{~Hz}, 1 \mathrm{H}, 2-\mathrm{H}_{\alpha, \mathrm{Cy}}\right), 2.04$ $\left(\mathrm{dd}, J=14.4,2.7 \mathrm{~Hz}, 1 \mathrm{H}, 2-\mathrm{H}_{\beta, \mathrm{Cy}}\right), 2.00(\mathrm{dt}, J=12.9$, $\left.3.6 \mathrm{~Hz}, 2 \mathrm{H}, 6-\mathrm{H}_{\alpha}\right), 1.79\left(\mathrm{t}, J=13.5 \mathrm{~Hz}, 1 \mathrm{H}, 6-\mathrm{H}_{\beta}\right) \mathrm{ppm}$; ${ }^{13} \mathrm{C} \mathrm{NMR}\left(75 \mathrm{MHz}, \mathrm{CDCl}_{3}\right): \delta=37.7(5-\mathrm{C}), 38.5\left(2-\mathrm{C}_{\mathrm{Cy}}\right)$, 41.5 (6-C), $64.3\left(\mathrm{OCH}_{2} \mathrm{CH}_{2} \mathrm{O}_{\text {acetal }}, 2^{\prime}-\mathrm{C}\right.$ or $\left.3^{\prime}-\mathrm{C}\right), 64.4\left(2^{\prime}-\mathrm{C}\right.$ or $\left.3^{\prime}-\mathrm{C}\right), 65.1\left(\mathrm{OCH}_{2} \mathrm{CH}_{2} \mathrm{O}_{\text {acetal }}\right), 69.7\left(3-\mathrm{C}_{\mathrm{Cy}}\right), 91.1$ (4$\left.\mathrm{C}_{\mathrm{Cy}}\right), 107.6(1-\mathrm{C}), 116.1\left(5-\mathrm{C}_{\mathrm{Ar}}\right), 117.6\left(8-\mathrm{C}_{\mathrm{Ar}}\right), 120.2$ (7$\left.\mathrm{C}_{\mathrm{Ar}}\right), 132.6\left(6-\mathrm{C}_{\mathrm{Ar}}\right), 140.3\left(8 \mathrm{a}-\mathrm{C}_{\mathrm{Ar}}\right), 141.9\left(4 \mathrm{a}-\mathrm{C}_{\mathrm{Ar}}\right) \mathrm{ppm}$; IR (KBr): $\bar{v}=3502,2980,2943,2887,1592,1551,1507$, $1458,1386,1362,1243,1125,1050,953,890,820$, $640 \mathrm{~cm}^{-1} ;[\alpha]_{\mathrm{D}}^{22}=+47.0^{\circ}(c=0.5, \mathrm{DMF})$.

(+)-3-Hydroxy-5-(8'-methoxy-2', $3^{\prime}$-dihydrobenzo[b][1,4]dioxin-6'-yl)-4-nitrocyclohexanone ethylene acetal $[(+)-26$, $\mathrm{C}_{17} \mathrm{H}_{21} \mathrm{NO}_{8}$ ] Yield: $75 \%$; white solid; m.p.: $178-180{ }^{\circ} \mathrm{C}$; ${ }^{1} \mathrm{H} \mathrm{NMR}\left(300 \mathrm{MHz}, \mathrm{CDCl}_{3}\right): \delta=6.40\left(\mathrm{~s}, 2 \mathrm{H}, 5-\mathrm{H}_{\mathrm{Ar}}\right.$ and7$\left.\mathrm{H}_{\mathrm{Ar}}\right), 4.71(\mathrm{dd}, J=12.0,2.7 \mathrm{~Hz}, 1 \mathrm{H}, 4-\mathrm{H}), 4.63(\mathrm{dq}$, $\left.J=9.9,3.0 \mathrm{~Hz}, 1 \mathrm{H}, 3-\mathrm{H}_{\mathrm{Cy}}\right), 4.28-4.22\left(\mathrm{~m}, 4 \mathrm{H}, \mathrm{OCH}_{2-}\right.$ $\left.\mathrm{CH}_{2} \mathrm{O}_{\text {benzodioxane }}\right), 4.09-3.92\left(\mathrm{~m}, 5 \mathrm{H}, \mathrm{OH}\right.$ and $\mathrm{OCH}_{2} \mathrm{CH}_{2-}$ $\left.\mathrm{O}_{\text {acetal }}\right), 3.86\left(\mathrm{~s}, 3 \mathrm{H}, \mathrm{OCH}_{3}\right), 3.74(\mathrm{td}, J=12.6,4.2 \mathrm{~Hz}, 1 \mathrm{H}$, 5-H), 2.19 (dt, $\left.J=14.7,3.0 \mathrm{~Hz}, 1 \mathrm{H}, 2-\mathrm{H}_{\alpha, \mathrm{Cy}}\right), 2.07-1.98$ $\left(\mathrm{m}, 2 \mathrm{H}, 2-\mathrm{H}_{\beta, \mathrm{Cy}}\right.$ and $\left.6-\mathrm{H}_{\alpha}\right), 1.80\left(\mathrm{t}, J=13.5 \mathrm{~Hz}, 1 \mathrm{H}, 6-\mathrm{H}_{\beta}\right)$ ppm; ${ }^{13} \mathrm{C} \mathrm{NMR} \mathrm{(75} \mathrm{MHz,} \mathrm{CDCl}_{3}$ ): $\delta=38.2$ (5-C), 38.4 (2$\left.\mathrm{C}_{\mathrm{Cy}}\right), 41.5(6-\mathrm{C}), 56.2\left(\mathrm{OCH}_{3}\right), 64.2\left(\mathrm{OCH}_{2} \mathrm{CH}_{2} \mathrm{O}_{\text {acetal }}\right)$, 
$64.3\left(2^{\prime}-\mathrm{C}\right.$ and $\left.3^{\prime}-\mathrm{C}\right), 65.1\left(\mathrm{OCH}_{2} \mathrm{CH}_{2} \mathrm{O}_{\text {acetal }}\right), 69.6\left(3-\mathrm{C}_{\mathrm{Cy}}\right)$, 91.0 (4-C), $104.0\left(7-\mathrm{C}_{\mathrm{Ar}}\right), 107.6(1-\mathrm{C}), 108.3\left(5-\mathrm{C}_{\mathrm{Ar}}\right)$, $131.8\left(8 \mathrm{a}-\mathrm{C}_{\mathrm{Ar}}\right), 132.4\left(6-\mathrm{C}_{\mathrm{Ar}}\right), 144.1\left(4 \mathrm{a}-\mathrm{C}_{\mathrm{Ar}}\right), 148.9(8-$ $\mathrm{C}_{\mathrm{Ar}}$ ) ppm; IR (KBr): $\bar{v}=3514,2974,2939,2895,1599$, $1544,1513,1460,1385,1352,1249,1127,1051,950$, $840 \mathrm{~cm}^{-1} ;[\alpha]_{\mathrm{D}}^{22}=+13.8^{\circ}\left(c=1, \mathrm{CHCl}_{3}\right)$.

\section{General procedure for the synthesis of amines $(+)-27$ and $(+)-28$}

Over a 10\% Pd/C catalyst (Selcat Q, $0.75 \mathrm{~g}), 2.53 \mathrm{~g} \mathrm{(+)-25}$ $(7.50 \mathrm{mmol})$ or $2.75 \mathrm{~g}(+)-26(7.50 \mathrm{mmol})$ was hydrogenated in $50 \mathrm{~cm}^{3} \mathrm{MeOH}$, in a $250 \mathrm{~cm}^{3}$ stainless steel autoclave equipped with a magnetic stirrer (stirring speed: $1100 \mathrm{rpm}$ ). The reduction was carried out at 12 bar and $60-80{ }^{\circ} \mathrm{C}$ for $6 \mathrm{~h}$. After the hydrogen uptake was finished, the catalyst was removed by filtration and the filtrate was concentrated in vacuo to afford (+)-27 or (+)-28.

\section{(+)-4-Amino-3-hydroxy-5-(2',3'-dihydrobenzo[b][1,4]-}

dioxin- $6^{\prime}$-yl)cyclohexanone ethylene acetal $[(+)-27$,

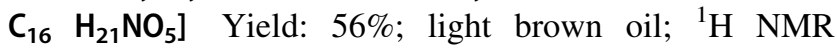
$\left(300 \mathrm{MHz}, \mathrm{CDCl}_{3}\right): \delta=6.81\left(\mathrm{~d}, J=8.4 \mathrm{~Hz}, 1 \mathrm{H}, 8-\mathrm{H}_{\mathrm{Ar}}\right)$, $6.71\left(\mathrm{~d}, J=1.2 \mathrm{~Hz}, 1 \mathrm{H}, 5-\mathrm{H}_{\mathrm{Ar}}\right), 6.67(\mathrm{dd}, J=8.4,1.8 \mathrm{~Hz}$, $\left.1 \mathrm{H}, 7-\mathrm{H}_{\mathrm{Ar}}\right), 4.23\left(\mathrm{~s}, 4 \mathrm{H}, \mathrm{OCH}_{2} \mathrm{CH}_{2} \mathrm{O}_{\text {benzodioxane }}\right), 4.06-3.85$ $\left(\mathrm{m}, 5 \mathrm{H}, 3-\mathrm{H}_{\mathrm{Cy}}\right.$ and $\left.\mathrm{OCH}_{2} \mathrm{CH}_{2} \mathrm{O}_{\text {acetal }}\right), 2.77-2.71(\mathrm{~m}, 2 \mathrm{H}, 4-$ $\mathrm{H}, 5-\mathrm{H}), 2.20\left(\mathrm{bs}, 2 \mathrm{H}, \mathrm{NH}_{2}\right), 2.11(\mathrm{dt}, J=14.1,2.7 \mathrm{~Hz}, 1 \mathrm{H}$, $\left.2-\mathrm{H}_{\alpha, \mathrm{Cy}}\right), 1.80\left(\mathrm{dd}, J=14.1,3.0 \mathrm{~Hz}, 1 \mathrm{H}, 2-\mathrm{H}_{\beta}, \mathrm{Cy}\right), 1.88-$ $1.77\left(\mathrm{~m}, 2 \mathrm{H}, 6-\mathrm{H}_{\alpha}\right.$ and $\left.6-\mathrm{H}_{\beta}\right) \mathrm{ppm} ;{ }^{13} \mathrm{C}$ NMR $(75 \mathrm{MHz}$, $\left.\mathrm{CDCl}_{3}\right): \delta=39.2\left(2-\mathrm{C}_{\mathrm{Cy}}\right), 41.6$ (6-C), 43.7 (5-C), 57.3 (4C), $64.0\left(\mathrm{OCH}_{2} \mathrm{CH}_{2} \mathrm{O}_{\text {acetal }}\right), 64.3\left(2^{\prime}-\mathrm{C}\right.$ and $\left.3^{\prime}-\mathrm{C}\right), 64.8$ $\left(\mathrm{OCH}_{2} \mathrm{CH}_{2} \mathrm{O}_{\text {acetal }}\right), 70.4\left(3-\mathrm{C}_{\mathrm{Cy}}\right), 108.8$ (1-C), 116.4 (8$\left.\mathrm{C}_{\mathrm{Ar}}\right), 117.4\left(5-\mathrm{C}_{\mathrm{Ar}}\right), 120.7\left(7-\mathrm{C}_{\mathrm{Ar}}\right), 135.4\left(6-\mathrm{C}_{\mathrm{Ar}}\right), 142.4$ $\left(8 \mathrm{a}-\mathrm{C}_{\mathrm{Ar}}\right), 143.6\left(4 \mathrm{a}-\mathrm{C}_{\mathrm{Ar}}\right) \mathrm{ppm}$; IR $(\mathrm{KBr}): \bar{v}=3503,2929$, 2880, 1589, 1508, 1433, 1371, 1289, 1245, 1110, 1067, $1002,948,888,815,747 \mathrm{~cm}^{-1} ;[\alpha]_{\mathrm{D}}^{22}=+15.5^{\circ}(c=1$, $\left.\mathrm{CHCl}_{3}\right)$.

(+)-4-Amino-3-hydroxy-5-(8'-methoxy-2', $3^{\prime}$-dihydrobenzo[b][1,4]dioxin- $6^{\prime}$-yl)cyclohexanone ethylene acetal $\left[(+)-28, \mathrm{C}_{17} \mathrm{H}_{23} \mathrm{NO}_{6}\right]$ Yield: $75 \%$; grey semi-solid; ${ }^{1} \mathrm{H}$ NMR $\left(300 \mathrm{MHz}, \mathrm{CDCl}_{3}\right): \delta=6.40(\mathrm{~d}, J=1.8 \mathrm{~Hz}, 1 \mathrm{H}, 5-$ $\mathrm{H}_{\mathrm{Ar}}$ or $\left.7-\mathrm{H}_{\mathrm{Ar}}\right), 6.35\left(\mathrm{~d}, J=1.2 \mathrm{~Hz}, 5-\mathrm{H}_{\mathrm{Ar}}\right.$ or $\left.7-\mathrm{H}_{\mathrm{Ar}}\right), 4.30-$ $4.24\left(\mathrm{~m}, 4 \mathrm{H}, \mathrm{OCH}_{2} \mathrm{CH}_{2} \mathrm{O}_{\text {benzodioxane }}\right), 4.07-3.87(\mathrm{~m}, 5 \mathrm{H}, 3-$ $\mathrm{H}_{\mathrm{Cy}}$ and $\left.\mathrm{OCH}_{2} \mathrm{CH}_{2} \mathrm{O}_{\text {acetal }}\right), 3.86\left(\mathrm{~s}, 3 \mathrm{H}, \mathrm{OCH}_{3}\right), 2.76-2.73$ (m, 2H, 4-H and 5-H), 2.12 (dt, $J=14.1,2.7 \mathrm{~Hz}, 1 \mathrm{H}, 2-$ $\mathrm{H}_{\alpha, \mathrm{Cy}}$ ), 2.07 (bs, 2H, $\mathrm{NH}_{2}$ ), $1.94(\mathrm{dd}, J=14.1,3.0 \mathrm{~Hz}, 1 \mathrm{H}$, $\left.2-\mathrm{H}_{\beta, \mathrm{Cy}}\right), 1.90-1.78\left(\mathrm{~m}, 2 \mathrm{H}, 6-\mathrm{H}_{\alpha}\right.$ and $\left.6-\mathrm{H}_{\beta}\right) \mathrm{ppm} ;{ }^{13} \mathrm{C}$ NMR $\left(75 \mathrm{MHz}, \mathrm{CDCl}_{3}\right): \delta=39.2\left(2-\mathrm{C}_{\mathrm{Cy}}\right), 41.6(6-\mathrm{C})$, 44.3 (5-C), $56.1\left(\mathrm{OCH}_{3}\right), 57.3(4-\mathrm{C}), 64.1\left(\mathrm{OCH}_{2} \mathrm{CH}_{2} \mathrm{O}_{\mathrm{ac}-}\right.$ etal $), 64.3\left(2^{\prime}-\mathrm{C}\right.$ or $\left.3^{\prime}-\mathrm{C}\right), 64.5\left(2^{\prime}-\mathrm{C}\right.$ or $\left.3^{\prime}-\mathrm{C}\right), 64.8\left(\mathrm{OCH}_{2-}\right.$ $\left.\mathrm{CH}_{2} \mathrm{O}_{\text {acetal }}\right), 70.5\left(3-\mathrm{C}_{\mathrm{Cy}}\right), 103.7\left(7-\mathrm{C}_{\mathrm{Ar}}\right), 108.9$ (1-C), $109.1\left(5-\mathrm{C}_{\mathrm{Ar}}\right), 131.9\left(6-\mathrm{C}_{\mathrm{Ar}}\right), 134.5\left(8 \mathrm{a}-\mathrm{C}_{\mathrm{Ar}}\right), 144.1(8-$ $\left.\mathrm{C}_{\mathrm{Ar}}\right), 149.0\left(4 \mathrm{a}-\mathrm{C}_{\mathrm{Ar}}\right) \mathrm{ppm}$; IR $(\mathrm{KBr}): \bar{v}=3503,2929,1598$,
1558, 1508, 1458, 1371, 1341, 1216, 1126, 1069, 952, $887 \mathrm{~cm}^{-1} ;[\alpha]_{\mathrm{D}}^{22}=+6.3^{\circ}(c=1$, methanol $)$.

\section{General procedure for the synthesis of carbamates $(+)-29$ and $(+)-30$}

Half of the required methyl chloroformate $\left(0.64 \mathrm{~cm}^{3}\right.$, $0.80 \mathrm{~g}, 8.47 \mathrm{mmol}), 3 \%$ aqueous $\mathrm{NaOH}$ solution $\left(20 \mathrm{~cm}^{3}\right)$, and subsequently the other half of methyl chloroformate $\left(0.64 \mathrm{~cm}^{3}, 0.80 \mathrm{~g}, 8.47 \mathrm{mmol}\right)$ were added to a solution of $2.54 \mathrm{~g} \mathrm{(+)-27}(8.27 \mathrm{mmol})$ or $2.79 \mathrm{~g}(+)-28(8.27 \mathrm{mmol})$ in $51 \mathrm{~cm}^{3}$ tetrahydrofuran. The reaction mixture was stirred rigorously at $\mathrm{rt}$ for $2 \mathrm{~h}$, then it was poured into $113 \mathrm{~cm}^{3}$ water and extracted with ethyl acetate $\left(4 \times 105 \mathrm{~cm}^{3}\right)$. The combined organic layer was washed with brine, dried over $\mathrm{Na}_{2} \mathrm{SO}_{4}$, and the solvent was evaporated in vacuo to give (+)-29 or (+)-30.

(+)-3-Hydroxy-4-methoxycarbonylamino-5-(2', $3^{\prime}$-dihydrobenzo[b][1,4]dioxin- $\left.6^{\prime}-y \mathrm{l}\right)$ cyclohexanone ethylene acetal $\left[(+)-29, \mathrm{C}_{18} \mathrm{H}_{23} \mathrm{NO}_{7}\right]$ Yield: $100 \%$; brown oil; ${ }^{1} \mathrm{H}$ NMR $\left(300 \mathrm{MHz}, \mathrm{CDCl}_{3}\right): \delta=6.78\left(\mathrm{~d}, J=8.1 \mathrm{~Hz}, 1 \mathrm{H}, 8-\mathrm{H}_{\mathrm{Ar}}\right)$, 6.72-6.68 (m, 2H, 5- $\mathrm{H}_{\mathrm{Ar}}$ and $\left.7-\mathrm{H}_{\mathrm{Ar}}\right), 4.98(\mathrm{~d}, J=9.0 \mathrm{~Hz}$, $1 \mathrm{H}, \mathrm{NH}), 4.22\left(\mathrm{~s}, 4 \mathrm{H}, \mathrm{OCH}_{2} \mathrm{CH}_{2} \mathrm{O}_{\text {benzodioxane }}\right.$ ), 4.15-4.08 $\left(\mathrm{m}, 1 \mathrm{H}, 3-\mathrm{H}_{\mathrm{Cy}}\right), 4.04-3.88\left(\mathrm{~m}, 4 \mathrm{H}, \mathrm{OCH}_{2} \mathrm{CH}_{2} \mathrm{O}_{\text {acetal }}\right), 3.81$ $(\mathrm{m}, 1 \mathrm{H}, 4-\mathrm{H}), 3.56(\mathrm{~d}, J=9.6 \mathrm{~Hz}, 1 \mathrm{H}, \mathrm{OH}), 3.50(\mathrm{~s}, 3 \mathrm{H}$, $\left.\mathrm{NHCOOCH}_{3}\right), 2.91(\mathrm{td}, J=11.7,3.6 \mathrm{~Hz}, 1 \mathrm{H}, 5-\mathrm{H}), 2.08$ $\left(\mathrm{dt}, J=14.4,2.7 \mathrm{~Hz}, 1 \mathrm{H}, 2-\mathrm{H}_{\alpha, \mathrm{Cy}}\right), 2.00(\mathrm{dd}, J=14.4$, $\left.2.7 \mathrm{~Hz}, 1 \mathrm{H}, 2-\mathrm{H}_{\beta, C y}\right), 1.91\left(\mathrm{dt}, J=13.2,3.3 \mathrm{~Hz}, 1 \mathrm{H}, 6-\mathrm{H}_{\alpha}\right)$, $1.84\left(\mathrm{t}, J=12.6 \mathrm{~Hz}, 1 \mathrm{H}, 6-\mathrm{H}_{\beta}\right) \mathrm{ppm} ;{ }^{13} \mathrm{C} \mathrm{NMR}(75 \mathrm{MHz}$, $\left.\mathrm{CDCl}_{3}\right): \delta=38.6\left(2-\mathrm{C}_{\mathrm{Cy}}\right), 40.8(5-\mathrm{C}), 42.5(6-\mathrm{C}), 51.9$ $\left(\mathrm{NHCOOCH}_{3}\right), 56.3$ (4-C), $64.2\left(\mathrm{OCH}_{2} \mathrm{CH}_{2} \mathrm{O}_{\text {acetal }}\right), 64.3$ $\left(2^{\prime}-\mathrm{C}\right.$ and $\left.3^{\prime}-\mathrm{C}\right), 64.9\left(\mathrm{OCH}_{2} \mathrm{CH}_{2} \mathrm{O}_{\text {acetal }}\right), 69.6\left(3-\mathrm{C}_{\mathrm{Cy}}\right)$, $108.5(1-\mathrm{C}), 116.6\left(5-\mathrm{C}_{\mathrm{Ar}}\right), 117.2\left(8-\mathrm{C}_{\mathrm{Ar}}\right), 120.4\left(7-\mathrm{C}_{\mathrm{Ar}}\right)$, $134.5\left(6-\mathrm{C}_{\mathrm{Ar}}\right), 142.3\left(8 \mathrm{a}-\mathrm{C}_{\mathrm{Ar}}\right), 143.3\left(4 \mathrm{a}-\mathrm{C}_{\mathrm{Ar}}\right), 156.4$ $\left(\mathrm{NHCOOCH}_{3}\right)$ ppm; IR (KBr): $\bar{v}=3471,3318,2950$, 2890, 1712, 1589, 1543, 1506, 1447, 1341, 1289, 1226, 1132, 1065, 993, 950, 919, 882,817, 777, $723 \mathrm{~cm}^{-1}$; $[\alpha]_{\mathrm{D}}^{22}=+17.8^{\circ}(c=1$, methanol $)$.

(+)-3-Hydroxy-4-methoxycarbonylamino-5-(8'-methoxy$2^{\prime}, 3^{\prime}$-dihydrobenzo[b][1,4]dioxin- $6^{\prime}$-yl)cyclohexanone ethylene acetal [(+)-30, $\left.\mathrm{C}_{19} \mathrm{H}_{25} \mathrm{NO}_{8}\right]$ Yield: $99 \%$; white solid (fluffy); m.p.: $84-90{ }^{\circ} \mathrm{C} ;{ }^{1} \mathrm{H}$ NMR $\left(300 \mathrm{MHz}, \mathrm{CDCl}_{3}\right.$ ): $\delta=6.39\left(\mathrm{~s}, 2 \mathrm{H}, 5-\mathrm{H}_{\mathrm{Ar}}\right.$ and $\left.7-\mathrm{H}_{\mathrm{Ar}}\right), 4.96(\mathrm{~d}, J=9.3 \mathrm{~Hz}, 1 \mathrm{H}$, $\mathrm{NH}), 4.30-4.22\left(\mathrm{~m}, 4 \mathrm{H}, \mathrm{OCH}_{2} \mathrm{CH}_{2} \mathrm{O}_{\text {benzodioxane }}\right), 4.15-4.08$ $\left(\mathrm{m}, 1 \mathrm{H}, 3-\mathrm{H}_{\mathrm{Cy}}\right), 4.05-3.91\left(\mathrm{~m}, 5 \mathrm{H}, 4-\mathrm{H}, \mathrm{OCH}_{2} \mathrm{CH}_{2} \mathrm{O}_{\text {acetal }}\right)$, $3.86\left(\mathrm{~s}, 3 \mathrm{H}, \mathrm{OCH}_{3}\right), 3.57(\mathrm{~d}, J=9.6 \mathrm{~Hz}, 1 \mathrm{H}, \mathrm{OH}), 3.51$ (s, $\left.3 \mathrm{H}, \mathrm{NHCOOCH}_{3}\right), 2.90(\mathrm{td}, J=11.4,3.0 \mathrm{~Hz}, 1 \mathrm{H}, 5-\mathrm{H})$, $2.09\left(\mathrm{dt}, J=14.4,3.0 \mathrm{~Hz}, 1 \mathrm{H}, 2-\mathrm{H}_{\alpha, \mathrm{Cy}}\right), 2.01(\mathrm{dd}, J=14.1$, $\left.3.3 \mathrm{~Hz}, 1 \mathrm{H}, 2-\mathrm{H}_{\beta, \mathrm{Cy}}\right), 1.92\left(\mathrm{dt}, J=13.2,3.3 \mathrm{~Hz}, 1 \mathrm{H}, 6-\mathrm{H}_{\alpha}\right)$, $1.83\left(\mathrm{t}, J=13.2 \mathrm{~Hz}, 1 \mathrm{H}, 6-\mathrm{H}_{\beta}\right) \mathrm{ppm} ;{ }^{13} \mathrm{C} \mathrm{NMR}(75 \mathrm{MHz}$, $\left.\mathrm{CDCl}_{3}\right): \delta=38.7\left(2-\mathrm{C}_{\mathrm{Cy}}\right), 41.2(5-\mathrm{C}), 42.8(6-\mathrm{C}), 51.9$

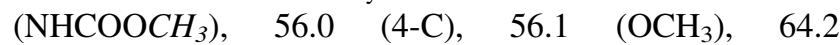


$\left(\mathrm{OCH}_{2} \mathrm{CH}_{2} \mathrm{O}_{\text {acetal }}, 2^{\prime}-\mathrm{C}\right.$ or $\left.3^{\prime}-\mathrm{C}\right), 64.5$ (2'-C or $\left.3^{\prime}-\mathrm{C}\right), 64.9$ $\left(\mathrm{OCH}_{2} \mathrm{CH}_{2} \mathrm{O}_{\text {acetal }}\right), 69.7\left(3-\mathrm{C}_{\mathrm{Cy}}\right), 103.2\left(7-\mathrm{C}_{\mathrm{Ar}}\right), 108.5$ (1C), $109.4\left(5-\mathrm{C}_{\mathrm{Ar}}\right), 131.8\left(8 \mathrm{a}-\mathrm{C}_{\mathrm{Ar}}\right), 133.6\left(6-\mathrm{C}_{\mathrm{Ar}}\right), 143.8(8-$ $\left.\mathrm{C}_{\mathrm{Ar}}\right), 148.7\left(4 \mathrm{a}-\mathrm{C}_{\mathrm{Ar}}\right), 156.4\left(\mathrm{NHCOOCH}_{3}\right) \mathrm{ppm}$; IR $(\mathrm{KBr})$ : $\bar{v}=3447,3340,2944,1714,1596,1536,1511,1457,1435$, $1340,1259,1225,1123,1080,1052,887 \mathrm{~cm}^{-1} ;[\alpha]_{\mathrm{D}}^{22-}$ $=+10.0^{\circ}\left(c=1, \mathrm{CHCl}_{3}\right)$.

\section{General procedure for the synthesis of enones $(-)-31$ and $(-)-32$}

A solution of $3.62 \mathrm{~g}(+)-29(9.91 \mathrm{mmol})$ or $3.92 \mathrm{~g}(+)-30$ (9.91 mmol) and $3.37 \mathrm{~g} p$-TsOH $(17.72 \mathrm{mmol})$ in $235 \mathrm{~cm}^{3}$ acetone was heated to reflux and stirred for $1 \mathrm{~h}$. After cooling to $\mathrm{rt}$, it was poured into $461 \mathrm{~cm}^{3}$ saturated $\mathrm{NaHCO}_{3}$ solution and extracted with ethyl acetate $\left(4 \times 138 \mathrm{~cm}^{3}\right)$. The combined organic layer was washed with brine, dried over $\mathrm{Na}_{2} \mathrm{SO}_{4}$, and the solvent was evaporated in vacuo to afford (-)-31 or (-)-32.

\section{(-)-6-(2', 3'-Dihydrobenzo[b][1,4]dioxin-6'-yl)-4-oxocyclo-}

hex-2-enyl)carbamic acid methyl ester [(-)-31, $\left.\mathrm{C}_{16} \mathrm{H}_{17} \mathrm{NO}_{5}\right]$ Yield: 93\%; white solid; m.p.: $153-155{ }^{\circ} \mathrm{C} ;{ }^{1} \mathrm{H}$ NMR $\left(300 \mathrm{MHz}, \mathrm{CDCl}_{3}\right): \delta=6.94\left(\mathrm{~d}, J=10.2 \mathrm{~Hz}, 1 \mathrm{H}, 2-\mathrm{H}_{\mathrm{Cy}}\right)$, $6.83\left(\mathrm{~d}, J=8.1 \mathrm{~Hz}, 1 \mathrm{H}, 8-\mathrm{H}_{\mathrm{Ar}}\right), 6.74(\mathrm{~d}, J=1.8 \mathrm{~Hz}, 1 \mathrm{H}, 5-$ $\left.\mathrm{H}_{\mathrm{Ar}}\right), 6.70\left(\mathrm{dd}, J=8.1,1.8 \mathrm{~Hz}, 1 \mathrm{H}, 7-\mathrm{H}_{\mathrm{Ar}}\right), 6.07(\mathrm{dd}$, $\left.J=10.2,2.4 \mathrm{~Hz}, 1 \mathrm{H}, 3-\mathrm{H}_{\mathrm{Cy}}\right), 4.82-4.71(\mathrm{~m}, 1 \mathrm{H}, \mathrm{NH})$, 4.61-4.56 (m, 1H, 1-H), $4.25\left(\mathrm{~s}, 4 \mathrm{H}, \mathrm{OCH}_{2} \mathrm{CH}_{2} \mathrm{O}\right), 3.60$ (s, $\left.3 \mathrm{H}, \mathrm{NHCOOCH}_{3}\right), 3.20-3.11(\mathrm{~m}, 1 \mathrm{H}, 6-\mathrm{H}), 2.68-2.65(\mathrm{~m}$, $2 \mathrm{H}, 5-\mathrm{H}_{\alpha}$ and $\left.5-\mathrm{H}_{\beta}\right) \mathrm{ppm} ;{ }^{13} \mathrm{C} \mathrm{NMR}\left(75 \mathrm{MHz}, \mathrm{CDCl}_{3}\right)$ : $\delta=44.9$ (5-C), 47.1 (6-C) $52.3(\mathrm{C}-1), 53.3\left(\mathrm{NHCOOCH}_{3}\right)$, $64.3\left(\mathrm{OCH}_{2} \mathrm{CH}_{2} \mathrm{O}\right), 116.0\left(5-\mathrm{C}_{\mathrm{Ar}}\right), 117.6\left(8-\mathrm{C}_{\mathrm{Ar}}\right), 120.2(7-$ $\left.\mathrm{C}_{\mathrm{Ar}}\right), 129.2\left(3-\mathrm{C}_{\mathrm{Cy}}\right), 132.9\left(6-\mathrm{C}_{\mathrm{Ar}}\right), 143.0\left(8 \mathrm{a}-\mathrm{C}_{\mathrm{Ar}}\right), 143.7$ $\left(4 \mathrm{a}-\mathrm{C}_{\mathrm{Ar}}\right), 151.6\left(2-\mathrm{C}_{\mathrm{Cy}}\right), 156.6\left(\mathrm{NHCOOCH}_{3}\right), 197.5(4-\mathrm{C})$ ppm; IR (KBr): $\bar{v}=3330,2984,2884,1698,1683,1591$, 1541, 1509, 1458, 1385, 1244, 1130, 1053, 927, 890, 817, $773 \mathrm{~cm}^{-1} ;[\alpha]_{\mathrm{D}}^{22}=-162.0^{\circ}(c=1$, acetone $)$.

(-)-6-(8'-Methoxy-2', $3^{\prime}$-dihydrobenzo[b][1,4]dioxin-6'-yl)-4oxocyclohex-2-enyl)carbamic acid methyl ester [(-)-32, $\mathrm{C}_{17} \mathrm{H}_{19} \mathrm{NO}_{6}$ ] Yield: 88\%; light brown oil; ${ }^{1} \mathrm{H}$ NMR $\left(300 \mathrm{MHz}, \mathrm{CDCl}_{3}\right): \delta=6.94\left(\mathrm{~d}, J=9.9,1 \mathrm{H}, 2-\mathrm{H}_{\mathrm{Cy}}\right), 6.40$ $\left(\mathrm{d}, J=1.8 \mathrm{~Hz}, 1 \mathrm{H}, 5-\mathrm{H}_{\mathrm{Ar}}\right.$ or $\left.7-\mathrm{H}_{\mathrm{Ar}}\right), 6.36(\mathrm{~d}, J=1.8 \mathrm{~Hz}$, $1 \mathrm{H}, 5-\mathrm{H}_{\mathrm{Ar}}$ or $\left.7-\mathrm{H}_{\mathrm{Ar}}\right), 6.07(\mathrm{dd}, J=10.2,2.4 \mathrm{~Hz}, 1 \mathrm{H}, 3-$ $\left.\mathrm{H}_{\mathrm{Cy}}\right), 4.82(\mathrm{~d}, J=7.5 \mathrm{~Hz}, 1 \mathrm{H}, \mathrm{NH}), 4.65-4.59(\mathrm{~m}, 1 \mathrm{H}, 1-$ $\mathrm{H}), 4.30-4.24\left(\mathrm{~m}, 4 \mathrm{H}, \mathrm{OCH}_{2} \mathrm{CH}_{2} \mathrm{O}\right), 3.86\left(\mathrm{~s}, 3 \mathrm{H}, \mathrm{OCH}_{3}\right)$ 3.61 (s, 3H, NHCOOCH$\left.H_{3}\right), 3.20-3.11(\mathrm{~m}, 1 \mathrm{H}, 6-\mathrm{H}), 2.68-$ $2.64\left(\mathrm{~m}, 2 \mathrm{H}, 5-\mathrm{H}_{\alpha}\right.$ and $\left.5-\mathrm{H}_{\beta}\right) \mathrm{ppm} ;{ }^{13} \mathrm{C} \mathrm{NMR}(75 \mathrm{MHz}$, $\left.\mathrm{CDCl}_{3}\right): \delta=45.0(5-\mathrm{C}), 47.6(6-\mathrm{C}), 52.3\left(\mathrm{NHCOOCH}_{3}\right)$, $53.1 \quad(1-\mathrm{C}), \quad 56.1 \quad\left(\mathrm{OCH}_{3}\right), \quad 64.2 \quad\left(\mathrm{OCH}_{2} \mathrm{CH}_{2} \mathrm{O}\right), 64.4$ $\left(\mathrm{OCH}_{2} \mathrm{CH}_{2} \mathrm{O}\right), 103.0\left(7-\mathrm{C}_{\mathrm{Ar}}\right), 108.8\left(5-\mathrm{C}_{\mathrm{Ar}}\right), 129.2\left(3-\mathrm{C}_{\mathrm{Cy}}\right)$, $132.0\left(8 \mathrm{a}-\mathrm{C}_{\mathrm{Ar}}\right), 132.4\left(6-\mathrm{C}_{\mathrm{Ar}}\right), 144.1\left(8 \mathrm{a}-\mathrm{C}_{\mathrm{Ar}}\right), 149.1(4 \mathrm{a}-$ $\left.\mathrm{C}_{\mathrm{Ar}}\right), 151.7\left(2-\mathrm{C}_{\mathrm{Cy}}\right), 156.3\left(\mathrm{NHCOOCH}_{3}\right), 197.5$ (4-C) ppm; IR (KBr): $\bar{v}=3341,2946,1699,1680,1598,1512$,
1462, 1385, 1342, 1242, 1127, 1050, 887, $652 \mathrm{~cm}^{-1}$; $[\alpha]_{\mathrm{D}}^{22}=-116.2^{\circ}\left(c=1, \mathrm{CHCl}_{3}\right)$.

\section{General procedure for the synthesis of enols (-)- 33 and $(-)-34$}

A solution of $2.63 \mathrm{~g} \mathrm{(-)-31}(8.67 \mathrm{mmol})$ or $2.89 \mathrm{~g} \mathrm{(-)-32}$ $(8.67 \mathrm{mmol})$ and $1.97 \mathrm{~g} \mathrm{CaCl}_{2}(17.75 \mathrm{mmol})$ in $264 \mathrm{~cm}^{3}$ methanol was stirred for $30 \mathrm{~min}$ at rt. Then, it was cooled to $0{ }^{\circ} \mathrm{C}$ and $0.49 \mathrm{~g} \mathrm{NaBH}_{4}(12.95 \mathrm{mmol})$ was added in one portion. It was further stirred at $0{ }^{\circ} \mathrm{C}$ for $30 \mathrm{~min}$, then poured into $372 \mathrm{~cm}^{3}$ water and extracted with ethyl acetate $\left(4 \times 188 \mathrm{~cm}^{3}\right)$. The combined organic layer was washed with brine, dried over $\mathrm{Na}_{2} \mathrm{SO}_{4}$, and the solvent was evaporated in vacuo. The residue was recrystallised from hexane/EtOAc (2:1) to give (-)-33 or (-)-34.

\section{(-)-6-(2',3'-Dihydrobenzo[b][1,4]dioxin-6'-yl)-4-hydroxycy-} clohex-2-enyl)carbamic acid methyl ester [(-)-33, $\mathrm{C}_{16} \mathrm{H}_{19} \mathrm{NO}_{5}$ ] Yield: $67 \%$; white solid; m.p.: $166-168{ }^{\circ} \mathrm{C}$; ${ }^{1} \mathrm{H} \mathrm{NMR}\left(300 \mathrm{MHz}, \mathrm{CDCl}_{3}\right): \delta=6.80(\mathrm{~d}, J=8.1 \mathrm{~Hz}, 1 \mathrm{H}$, $\left.8-\mathrm{H}_{\mathrm{Ar}}\right), 6.72\left(\mathrm{~d}, J=1.8 \mathrm{~Hz}, 1 \mathrm{H}, 5-\mathrm{H}_{\mathrm{Ar}}\right), 6.68(\mathrm{dd}, J=8.1$, $\left.1.8 \mathrm{~Hz}, 1 \mathrm{H}, 7-\mathrm{H}_{\mathrm{Ar}}\right), 5.81\left(\mathrm{dd}, J=10.2,1.2 \mathrm{~Hz}, 1 \mathrm{H}, 3-\mathrm{H}_{\mathrm{Cy}}\right)$, $5.75\left(\mathrm{~d}, J=10.2 \mathrm{~Hz}, 1 \mathrm{H}, 2-\mathrm{H}_{\mathrm{Cy}}\right), 4.60-4.50(\mathrm{~m}, 1 \mathrm{H}, \mathrm{NH})$, 4.50-4.38 (m, 1H, 4-H), 4.34-4.27 (m, 1H, 1-H), 4.24 (s, $4 \mathrm{H}, \mathrm{OCH}_{2} \mathrm{CH}_{2} \mathrm{O}$ ), 3.55 (s, $\left.3 \mathrm{H}, \mathrm{NHCOOCH}_{3}\right), 2.65-2.57$ $(\mathrm{m}, 1 \mathrm{H}, 6-\mathrm{H}), 2.24\left(\mathrm{dd}, J=12.0,5.4 \mathrm{~Hz}, 1 \mathrm{H}, 5-\mathrm{H}_{\beta}\right), 1.80$ $\left(\mathrm{td}, J=12.9,9.9 \mathrm{~Hz}, 1 \mathrm{H}, 5-\mathrm{H}_{\alpha}\right) \mathrm{ppm} ;{ }^{13} \mathrm{C} \mathrm{NMR}(75 \mathrm{MHz}$, $\left.\mathrm{CDCl}_{3}\right): \delta=40.6(5-\mathrm{C}), 45.7$ (6-C), $52.0\left(\mathrm{NHCOOCH}_{3}\right)$, 53.5 (1-C), $64.3\left(\mathrm{OCH}_{2} \mathrm{CH}_{2} \mathrm{O}\right), 67.8(4-\mathrm{C}), 116.0\left(5-\mathrm{C}_{\mathrm{Ar}}\right)$, $117.3\left(8-\mathrm{C}_{\mathrm{Ar}}\right), 120.2\left(7-\mathrm{C}_{\mathrm{Ar}}\right), 131.1\left(2-\mathrm{C}_{\mathrm{Cy}}\right), 132.4\left(3-\mathrm{C}_{\mathrm{Cy}}\right)$, $135.1\left(6-\mathrm{C}_{\mathrm{Ar}}\right), 142.5\left(8 \mathrm{a}-\mathrm{C}_{\mathrm{Ar}}\right), 143.5\left(4 \mathrm{a}-\mathrm{C}_{\mathrm{Ar}}\right), 156.5$ $\left(\mathrm{NHCOOCH}_{3}\right)$ ppm; IR (KBr): $\bar{v}=3329,2926,2876$, 1687, 1592, 1533, 1509, 1457, 1435, 1315, 1290, 1126, 1102, 1049, 928, 885, 759, $634 \mathrm{~cm}^{-1}$; HPLC: Chiralpack ${ }^{\circledR}$ OD (hexane $/ i-\mathrm{PrOH}=8: 2$, flow rate $2.0 \mathrm{~cm}^{3} \mathrm{~min}^{-1}$, $\left.256 \mathrm{~nm}, 20^{\circ} \mathrm{C}\right), \quad t_{(-)}: 23 \mathrm{~min}, \quad t_{(+)}: 30 \mathrm{~min} ; \quad[\alpha]_{\mathrm{D}}^{22-}$ $=-129.0^{\circ}\left(c=1, \mathrm{CHCl}_{3}\right)$; ee $>99 \%$.

(-)-6-(8'-Methoxy-2', $3^{\prime}$-dihydrobenzo[b][1,4]dioxin-6'-yl)-4hydroxycyclohex-2-enyl)carbamic acid methyl ester [(-)34, $\mathrm{C}_{17} \mathrm{H}_{21} \mathrm{NO}_{6}$ ] Yield: $79 \%$; white solid; m.p.: $166{ }^{\circ} \mathrm{C} ;{ }^{1} \mathrm{H}$ NMR $\left(300 \mathrm{MHz}, \mathrm{CDCl}_{3}\right): \delta=6.39(\mathrm{~d}, J=1.8 \mathrm{~Hz}, 1 \mathrm{H}$, $5-\mathrm{H}_{\mathrm{A}}$ or $\left.7-\mathrm{H}_{\mathrm{Ar}}\right), 6.36\left(\mathrm{~d}, J=1.8 \mathrm{~Hz}, 1 \mathrm{H}, 5-\mathrm{H}_{\mathrm{A}}\right.$ or $\left.7-\mathrm{H}_{\mathrm{Ar}}\right)$, $5.82\left(\mathrm{dd}, \quad J=10.2, \quad 1.2 \mathrm{~Hz}, \quad 1 \mathrm{H}, \quad 3-\mathrm{H}_{\mathrm{Cy}}\right), 5.76(\mathrm{~d}$, $\left.J=10.8 \mathrm{~Hz}, \quad 1 \mathrm{H}, \quad 2-\mathrm{H}_{\mathrm{Cy}}\right), \quad 4.60-4.50 \quad(\mathrm{~m}, \quad 1 \mathrm{H}, \quad \mathrm{NH})$, 4.50-4.40 (m, 1H, 4-H), 4.24-4.30 (m, 5H, 1-H and $\left.\mathrm{OCH}_{2} \mathrm{CH}_{2} \mathrm{O}\right), \quad 3.86 \quad\left(\mathrm{~s}, \quad 3 \mathrm{H}, \quad \mathrm{OCH}_{3}\right) 3.56 \quad(\mathrm{~s}, \quad 3 \mathrm{H}$, $\mathrm{NHCOOCH}_{3}$ ), 2.66-2.56 (m, 1H, 6-H), 2.26 (dd, $J=12.3$, $\left.5.4 \mathrm{~Hz}, 1 \mathrm{H}, 5-\mathrm{H}_{\beta}\right), 1.80\left(\mathrm{td}, J=12.9,9.9 \mathrm{~Hz}, 1 \mathrm{H}, 5-\mathrm{H}_{\alpha}\right)$ ppm; ${ }^{13} \mathrm{C}$ NMR (75 MHz, $\mathrm{CDCl}_{3}$ ): $\delta=40.0$ (5-C), 46.1 (6C), $52.1\left(\mathrm{NHCOOCH}_{3}\right), 53.7(1-\mathrm{C}), 56.1\left(\mathrm{OCH}_{3}\right), 64.3$ $\left(\mathrm{OCH}_{2} \mathrm{CH}_{2} \mathrm{O}\right), 64.5\left(\mathrm{OCH}_{2} \mathrm{CH}_{2} \mathrm{O}\right), 67.7$ (4-C), 103.1 (7$\left.\mathrm{C}_{\mathrm{Ar}}\right), 108.9\left(5-\mathrm{C}_{\mathrm{Ar}}\right), 131.1\left(2-\mathrm{C}_{\mathrm{Cy}}\right), 132.4\left(3-\mathrm{C}_{\mathrm{Cy}}\right), 133.7$ 
$\left(6-\mathrm{C}_{\mathrm{Ar}}\right), 134.1\left(8 \mathrm{a}-\mathrm{C}_{\mathrm{Ar}}\right), 143.9\left(8-\mathrm{C}_{\mathrm{Ar}}\right), 148.9\left(4 \mathrm{a}-\mathrm{C}_{\mathrm{Ar}}\right)$, $156.4\left(\mathrm{NHCOOCH}_{3}\right)$ ppm; IR $(\mathrm{KBr}): \bar{v}=3333,2930$, 1693, 1599, 1539, 1512, 1457, 1341, 1239, 1129, 1049, 887, $655 \mathrm{~cm}^{-1}$; HPLC: Chiralpack ${ }^{\circledR}$ OD (hexane $/ i-$ $\mathrm{PrOH}=8: 2$, flow rate $2.0 \mathrm{~cm}^{3} \mathrm{~min}^{-1}, 256 \mathrm{~nm}, 20^{\circ} \mathrm{C}$ ), $t_{(-)}: 30 \mathrm{~min}, t_{(+)}: 25 \mathrm{~min} ;[\alpha]_{\mathrm{D}}^{22}=-107.8^{\circ} \quad(c=1$, $\left.\mathrm{CHCl}_{3}\right) ; e e>99 \%$.

\section{General procedure for the Mitsunobu reaction}

$0.74 \mathrm{~cm}^{3}$ diethyl azodicarboxylate $(0.82 \mathrm{~g}, 4.71 \mathrm{mmol})$ in $2.6 \mathrm{~cm}^{3}$ anhydrous THF at $0{ }^{\circ} \mathrm{C}$ was added dropwise to a solution of $1.09 \mathrm{~g}(-)-33(3.58 \mathrm{mmol})$ or $1.20 \mathrm{~g}(-)-34$ $(3.58 \mathrm{mmol})$ and $1.15 \mathrm{~g}$ triphenylphosphine $(4.38 \mathrm{mmol})$ in $56 \mathrm{~cm}^{3}$ anhydrous THF, and the mixture was stirred for $10 \mathrm{~min}$. Then $0.47 \mathrm{~g}$ benzoic acid $(3.85 \mathrm{mmol})$ was also added, and the reaction mixture was stirred at $0{ }^{\circ} \mathrm{C}$ for $2 \mathrm{~h}$, then heated to $45-50{ }^{\circ} \mathrm{C}$ and further stirred for $5 \mathrm{~h}$. The solvent was evaporated in vacuo and the residue was purified as specified.

\section{(+)-5-(2',3'-Dihydrobenzo[b][1,4]dioxin-6'-yl)-4-(methoxy-} carbonylamino)cyclohex-2-enyl benzoate $[(+)-35$, $\mathrm{C}_{23} \mathrm{H}_{23} \mathrm{NO}_{6}$ ] It was purified by column chromatography $\left(\mathrm{CH}_{2} \mathrm{Cl}_{2}\right.$ /acetone, 20:1) to afford a pale yellow solid. Yield: 77\%; m.p.: 58-63 ${ }^{\circ} \mathrm{C} ; R_{f}=0.51\left(\mathrm{CHCl}_{3} /\right.$ methanol, 100:1); ${ }^{1} \mathrm{H}$ NMR $\left(300 \mathrm{MHz}, \mathrm{CDCl}_{3}\right): \delta=8.05(\mathrm{~d}, J=7.2 \mathrm{~Hz}, 2 \mathrm{H}$, $2-\mathrm{H}_{\mathrm{Bz}}$ and $\left.6-\mathrm{H}_{\mathrm{Bz}}\right), 7.57\left(\mathrm{t}, J=7.2 \mathrm{~Hz}, 1 \mathrm{H}, 4-\mathrm{H}_{\mathrm{Bz}}\right), 7.45(\mathrm{t}$, $J=7.2 \mathrm{~Hz}, 2 \mathrm{H}, 3-\mathrm{H}_{\mathrm{Bz}}$ and $\left.5-\mathrm{H}_{\mathrm{Bz}}\right), 6.81(\mathrm{~d}, J=8.4 \mathrm{~Hz}, 1 \mathrm{H}$, $\left.8-\mathrm{H}_{\mathrm{Ar}}\right), 6.76\left(\mathrm{~d}, J=1.8 \mathrm{~Hz}, 1 \mathrm{H}, 5-\mathrm{H}_{\mathrm{Ar}}\right), 6.72(\mathrm{dd}$, $\left.J=8.4 \mathrm{~Hz}, 7-\mathrm{H}_{\mathrm{Ar}}\right), 6.08-5.99\left(\mathrm{~m}, 2 \mathrm{H}, 2-\mathrm{H}_{\mathrm{Cy}}\right.$ and $\left.3-\mathrm{H}_{\mathrm{Cy}}\right)$, 5.53-5.48 (m, 1H, 1-H), 4.67-4.54 (m, 1H, NH), 4.41-4.29 $(\mathrm{m}, 1 \mathrm{H}, 4-\mathrm{H}), 4.24\left(\mathrm{~s}, 4 \mathrm{H}, \mathrm{OCH}_{2} \mathrm{CH}_{2} \mathrm{O}\right), 3.58(\mathrm{~s}, 3 \mathrm{H}$, $\mathrm{NHCOOCH}_{3}$ ), 2.93-2.83 (m, 1H, 5-H), 2.19-2.15 (m, 2H, 6- $\mathrm{H}_{\alpha}$ and $\left.6-\mathrm{H}_{\beta}\right)$ ppm; ${ }^{13} \mathrm{C} \mathrm{NMR}\left(75 \mathrm{MHz}, \mathrm{CDCl}_{3}\right)$ : $\delta=38.0$ (6-C), 42.9 (5-C), $52.1\left(\mathrm{NHCOOCH}_{3}\right), 53.2$ (4-C), $64.3\left(\mathrm{OCH}_{2} \mathrm{CH}_{2} \mathrm{O}\right), 64.4\left(\mathrm{OCH}_{2} \mathrm{CH}_{2} \mathrm{O}\right), 66.8(1-\mathrm{C}), 116.3$ $\left(5-\mathrm{C}_{\mathrm{Ar}}\right), 117.4\left(8-\mathrm{C}_{\mathrm{Ar}}\right), 120.3\left(7-\mathrm{C}_{\mathrm{Ar}}\right), 125.5\left(2-\mathrm{C}_{\mathrm{Cy}}\right), 128.4$ $\left(3-\mathrm{C}_{\mathrm{Bz}}\right.$ and $\left.5-\mathrm{C}_{\mathrm{Bz}}\right), 129.6\left(2-\mathrm{C}_{\mathrm{Bz}}\right.$ and $\left.6-\mathrm{C}_{\mathrm{Bz}}\right), 130.3\left(1-\mathrm{C}_{\mathrm{Bz}}\right)$, $133.0\left(4-\mathrm{C}_{\mathrm{Bz}}\right), 135.1\left(6-\mathrm{C}_{\mathrm{Ar}}\right), 135.8\left(3-\mathrm{C}_{\mathrm{Cy}}\right), 141.2(8 \mathrm{a}-$ $\left.\mathrm{C}_{\mathrm{Ar}}\right), 143.5\left(4 \mathrm{a}-\mathrm{C}_{\mathrm{Ar}}\right), 156.4\left(\mathrm{NHCOOCH}_{3}\right), 165.9(\mathrm{PhCO})$ ppm; IR (KBr): $\bar{v}=3356,3036,2947,1716,1591,1510$, $1452,1315,1271,1109,1053,1025,953,896,810$, $713 \mathrm{~cm}^{-1} ;[\alpha]_{\mathrm{D}}^{22}=+82.5^{\circ}\left(c=1, \mathrm{CHCl}_{3}\right)$.

(+)-5-(8'-Methoxy-2', $3^{\prime}$-dihydrobenzo[b][1,4]dioxin-6'-yl)-4(methoxycarbonylamino)cyclohex-2-enyl benzoate $[(+)-36$, $\mathrm{C}_{24} \mathrm{H}_{25} \mathrm{NO}_{7}$ ] It was isolated by column chromatography $\left(\mathrm{CHCl}_{3}\right.$ /acetone, $\left.20: 1\right)$ to give a white solid. Yield: $77 \%$; m.p.: $66-71{ }^{\circ} \mathrm{C} ; R_{f}=0.35\left(\mathrm{CHCl}_{3} /\right.$ methanol, $\left.100: 1\right) ;{ }^{1} \mathrm{H}$ NMR $\left(300 \mathrm{MHz}, \mathrm{CDCl}_{3}\right): \delta=8.05(\mathrm{~d}, J=6.9 \mathrm{~Hz}, 2 \mathrm{H}, 2-$ $\mathrm{H}_{\mathrm{Bz}}$ and $\left.6-\mathrm{H}_{\mathrm{Bz}}\right), 7.58\left(\mathrm{tt}, J=7.2,1.2 \mathrm{~Hz}, 1 \mathrm{H}, 4-\mathrm{H}_{\mathrm{Bz}}\right), 7.45$ $\left(\mathrm{t}, J=7.5 \mathrm{~Hz}, 2 \mathrm{H}, 3-\mathrm{H}_{\mathrm{B}}\right.$ and $\left.5-\mathrm{H}_{\mathrm{Bz}}\right), 6.43(\mathrm{~d}, J=1.8 \mathrm{~Hz}$,
$1 \mathrm{H}, 5-\mathrm{H}_{\mathrm{Ar}}$ or $\left.7-\mathrm{H}_{\mathrm{Ar}}\right), 6.40\left(\mathrm{~d}, J=1.8 \mathrm{~Hz}, 1 \mathrm{H}, 5-\mathrm{H}_{\mathrm{Ar}}\right.$ or $7-$ $\left.\mathrm{H}_{\mathrm{Ar}}\right), 6.08-5.99\left(\mathrm{~m}, 2 \mathrm{H}, 2-\mathrm{H}_{\mathrm{Cy}}\right.$ and $\left.3-\mathrm{H}_{\mathrm{Cy}}\right), 5.54-5.49(\mathrm{~m}$, $1 \mathrm{H}, 1-\mathrm{H}), 4.68-4.65(\mathrm{~m}, 1 \mathrm{H}, \mathrm{NH}), 4.43-4.33(\mathrm{~m}, 1 \mathrm{H}, 4-\mathrm{H})$, 4.30-4.24 (m, $\left.4 \mathrm{H}, \mathrm{OCH}_{2} \mathrm{CH}_{2} \mathrm{O}\right), 3.87\left(\mathrm{~s}, 3 \mathrm{H}, \mathrm{OCH}_{3}\right), 3.58$ (s, 3H, $\left.\mathrm{NHCOOCH}_{3}\right), 2.92-2.84(\mathrm{~m}, 1 \mathrm{H}, 5-\mathrm{H}), 2.20-2.16$ (m, 2H, 6- $\mathrm{H}_{\alpha}$ and 6- $\mathrm{H}_{\beta}$ ) ppm; ${ }^{13} \mathrm{C} \mathrm{NMR} \mathrm{(75} \mathrm{MHz}, \mathrm{CDCl}_{3}$ ): $\delta=36.0(6-\mathrm{C}), 42.0(5-\mathrm{C}), 52.1\left(\mathrm{NHCOOCH}_{3}\right), 53.4$ (4-C), $56.2\left(\mathrm{OCH}_{3}\right), 64.3\left(\mathrm{OCH}_{2} \mathrm{CH}_{2} \mathrm{O}\right), 64.5\left(\mathrm{OCH}_{2} \mathrm{CH}_{2} \mathrm{O}\right), 66.7$ (1-C), $103.2\left(7-\mathrm{C}_{\mathrm{Ar}}\right), 109.2\left(5-\mathrm{C}_{\mathrm{Ar}}\right), 125.6\left(2-\mathrm{C}_{\mathrm{Cy}}\right), 128.4$ $\left(3-\mathrm{C}_{\mathrm{Bz}}\right.$ and $\left.5-\mathrm{C}_{\mathrm{Bz}}\right), 129.6\left(2-\mathrm{C}_{\mathrm{Bz}}\right.$ and $\left.6-\mathrm{C}_{\mathrm{Bz}}\right), 130.3\left(1-\mathrm{C}_{\mathrm{Bz}}\right)$, $132.0\left(6-\mathrm{C}_{\mathrm{Ar}}\right), 133.0\left(4-\mathrm{C}_{\mathrm{Bz}}\right), 134.1\left(8 \mathrm{a}-\mathrm{C}_{\mathrm{Ar}}\right), 135.8(3-$ $\left.\mathrm{C}_{\mathrm{Cy}}\right), 143.9\left(8-\mathrm{C}_{\mathrm{Ar}}\right), 148.9\left(4 \mathrm{a}-\mathrm{C}_{\mathrm{Ar}}\right), 156.4\left(\mathrm{NHCOOCH}_{3}\right)$, 165.9 (PhCO) ppm; IR (KBr): $\bar{v}=3362,2931,1716,1599$, $1511,1453,1362,1340,1271,1129,1053,1025,887$, $649 \mathrm{~cm}^{-1} ;[\alpha]_{\mathrm{D}}^{22}=+90.3^{\circ}\left(c=1, \mathrm{CHCl}_{3}\right)$.

\section{General procedure for synthesis of cis-diols $(+)-$ 37 and $(+)-38$}

To a solution of $1.15 \mathrm{~g}(+)-\mathbf{3 5}(2.80 \mathrm{mmol})$ or $1.23 \mathrm{~g}(+)$ $36(2.80 \mathrm{mmol})$ in a mixture of $17 \mathrm{~cm}^{3}$ tetrahydrofuran and $2.8 \mathrm{~cm}^{3} \quad$ water, $\quad 0.71 \mathrm{~g} N$-methylmorpholine- $N$-oxide (6.06 mmol) and subsequently $1.22 \mathrm{~cm}^{3} 4 \%$ aqueous $\mathrm{OsO}_{4}$ solution $(48.6 \mathrm{mg}, 0.19 \mathrm{mmol})$ under argon were added. The mixture was stirred at $\mathrm{rt}$ for $24 \mathrm{~h}$, then it was poured into $112 \mathrm{~cm}^{3}$ saturated $\mathrm{Na}_{2} \mathrm{~S}_{2} \mathrm{O}_{3}$ solution and extracted with ethyl acetate $\left(4 \times 60 \mathrm{~cm}^{3}\right)$. The combined organic layer was washed with brine, dried over $\mathrm{Na}_{2} \mathrm{SO}_{4}$, and the solvent was evaporated in vacuo to afford $(+)-37$ or $(+)-$ 38.

\section{(+)-5-(2',3'-Dihydrobenzo[b][1,4]dioxin-6'-yl)-2,3-dihy-} droxy-4-(methoxycarbonylamino)cyclohexyl benzoate $[(+)$ 37, $\mathrm{C}_{23} \mathrm{H}_{25} \mathrm{NO}_{8}$ ] Yield: $99 \%$; white solid (fluffy); m.p.: $92{ }^{\circ} \mathrm{C} ; \quad{ }^{1} \mathrm{H}$ NMR $\left(300 \mathrm{MHz}, \mathrm{CDCl}_{3}\right): \delta=8.03 \quad(\mathrm{~d}$, $J=7.5 \mathrm{~Hz}, 2 \mathrm{H}, 2-\mathrm{H}_{\mathrm{Bz}}$ and $\left.6-\mathrm{H}_{\mathrm{Bz}}\right), 7.60(\mathrm{t}, J=7.5 \mathrm{~Hz}, 1 \mathrm{H}$, $\left.4-\mathrm{H}_{\mathrm{Bz}}\right), 7.47\left(\mathrm{t}, J=7.5 \mathrm{~Hz}, 2 \mathrm{H}, 3-\mathrm{H}_{\mathrm{Bz}}\right.$ and $\left.5-\mathrm{H}_{\mathrm{Bz}}\right), 6.80(\mathrm{~d}$, $\left.J=8.1 \mathrm{~Hz}, 1 \mathrm{H}, 8-\mathrm{H}_{\mathrm{Ar}}\right), 6.73\left(\mathrm{~d}, J=1.8 \mathrm{~Hz}, 1 \mathrm{H}, 5-\mathrm{H}_{\mathrm{Ar}}\right)$, $6.68\left(\mathrm{dd}, J=8.1,1.8 \mathrm{~Hz}, 1 \mathrm{H}, 5-\mathrm{H}_{\mathrm{Ar}}\right), 5.41(\mathrm{q}, J=2.7 \mathrm{~Hz}$, $1 \mathrm{H}, 1-\mathrm{H}), 4.71-4.62(\mathrm{~m}, 1 \mathrm{H}, \mathrm{NH}), 4.25-4.18(\mathrm{~m}, 5 \mathrm{H}$, $\mathrm{OCH}_{2} \mathrm{CH}_{2} \mathrm{O}$ and $\left.2-\mathrm{H}_{\mathrm{Cy}}\right), 4.06-3.95\left(\mathrm{~m}, 2 \mathrm{H}, 3-\mathrm{H}_{\mathrm{Cy}}\right.$ and 4$\mathrm{H}), 3.58\left(\mathrm{~s}, 3 \mathrm{H}, \mathrm{NHCOOCH}_{3}\right), 2.82$ (ddd, $J=13.5,11.1$, $3.0 \mathrm{~Hz}, 1 \mathrm{H}, 5-\mathrm{H}), 2.30(\mathrm{ddd}, J=15.9,11.4,2.4 \mathrm{~Hz}, 1 \mathrm{H}, 6-$ $\left.\mathrm{H}_{\beta}\right), 2.02\left(\mathrm{dt}, J=14.7,3.3 \mathrm{~Hz}, 1 \mathrm{H}, 6-\mathrm{H}_{\alpha}\right) \mathrm{ppm} ;{ }^{13} \mathrm{C} \mathrm{NMR}$ $\left(75 \mathrm{MHz}, \mathrm{CDCl}_{3}\right): \delta=32.9 \quad(6-\mathrm{C}), 41.9 \quad(5-\mathrm{C}), \quad 52.6$ $\left(\mathrm{NHCOOCH}_{3}\right), 55.7$ (4-C), $64.3\left(\mathrm{OCH}_{2} \mathrm{CH}_{2} \mathrm{O}\right), 70.1$ $\left.\mathrm{C}_{\mathrm{Cy}}\right), 71.3(1-\mathrm{C}), 74.4\left(3-\mathrm{C}_{\mathrm{Cy}}\right), 116.3\left(5-\mathrm{C}_{\mathrm{Ar}}\right), 117.6$ (8$\left.\mathrm{C}_{\mathrm{Ar}}\right), 120.4\left(7-\mathrm{C}_{\mathrm{Ar}}\right), 128.5\left(3-\mathrm{C}_{\mathrm{Bz}}\right.$ and $\left.5-\mathrm{C}_{\mathrm{Bz}}\right), 129.6\left(2-\mathrm{C}_{\mathrm{Bz}}\right.$ and $\left.6-\mathrm{C}_{\mathrm{BZ}}\right), 130.0\left(1-\mathrm{C}_{\mathrm{Bz}}\right), 133.3\left(4-\mathrm{C}_{\mathrm{Bz}}\right), 133.8\left(6-\mathrm{C}_{\mathrm{Ar}}\right)$, $142.7\left(8 \mathrm{a}-\mathrm{C}_{\mathrm{Ar}}\right), 143.7\left(4 \mathrm{a}-\mathrm{C}_{\mathrm{Ar}}\right), 158.9\left(\mathrm{NHCOOCH}_{3}\right)$, 165.1 (PhCO) ppm; IR (KBr): $\bar{v}=3421,2928,2877,1716$, 
1591, 1541, 1509, 1456, 1374, 1338, 1273, 1113, 1070, $1045,887,714 \mathrm{~cm}^{-1} ;[\alpha]_{\mathrm{D}}^{22}=+65.2^{\circ}\left(c=1, \mathrm{CHCl}_{3}\right)$.

(+)-2,3-Dihydroxy-5-(8'-methoxy-2', $3^{\prime}$-dihydrobenzo[b][1,4]dioxin- $6^{\prime}$-yl)-4-(methoxycarbonylamino)cyclohexyl benzoate $\left[(+)-38, \mathrm{C}_{24} \mathrm{H}_{27} \mathrm{NO}_{9}\right]$ Yield: $100 \%$; white solid (fluffy); m.p.: $\quad 112-120{ }^{\circ} \mathrm{C} ;{ }^{1} \mathrm{H} \quad \mathrm{NMR}$ $\left(300 \mathrm{MHz}, \mathrm{CDCl}_{3}\right): \delta=8.04(\mathrm{dd}, J=7.2,1.5 \mathrm{~Hz}, 2 \mathrm{H}, 2-$ $\mathrm{H}_{\mathrm{Bz}}$ and $\left.6-\mathrm{H}_{\mathrm{Bz}}\right), 7.61\left(\mathrm{t}, J=7.2 \mathrm{~Hz}, 1 \mathrm{H}, 4-\mathrm{H}_{\mathrm{Bz}}\right), 7.48(\mathrm{t}$, $J=7.5 \mathrm{~Hz}, 2 \mathrm{H}, 3-\mathrm{H}_{\mathrm{Bz}}$ and $\left.5-\mathrm{H}_{\mathrm{Bz}}\right), 6.39(\mathrm{~d}, J=1.8 \mathrm{~Hz}, 1 \mathrm{H}$, $5-\mathrm{H}_{\mathrm{Ar}}$ or $\left.7-\mathrm{H}_{\mathrm{Ar}}\right), 6.35\left(\mathrm{~d}, J=1.8 \mathrm{~Hz}, 1 \mathrm{H}, 5-\mathrm{H}_{\mathrm{Ar}}\right.$ or $\left.7-\mathrm{H}_{\mathrm{Ar}}\right)$, $5.43(\mathrm{q}, J=3.0 \mathrm{~Hz}, 1 \mathrm{H}, 1-\mathrm{H}), 4.65-4.64(\mathrm{~m}, 1 \mathrm{H}, \mathrm{NH}), 4.56$ (bs, $1 \mathrm{H}, \mathrm{OH}), 4.30-4.23\left(\mathrm{~m}, 4 \mathrm{H}, \mathrm{OCH}_{2} \mathrm{CH}_{2} \mathrm{O}\right), 4.21-4.18$ $\left(\mathrm{m}, 1 \mathrm{H}, 2-\mathrm{H}_{\mathrm{Cy}}\right), 4.06-3.95\left(\mathrm{~m}, 2 \mathrm{H}, 3-\mathrm{H}_{\mathrm{Cy}}\right.$ and $\left.4-\mathrm{H}\right), 3.86(\mathrm{~s}$, $\left.3 \mathrm{H}, \mathrm{OCH}_{3}\right), 3.59$ (s, $\left.3 \mathrm{H}, \mathrm{NHCOOCH}_{3}\right), 3.18(\mathrm{bs}, 1 \mathrm{H}, \mathrm{OH})$, 2.80 (ddd, $J=12.9,10.8,3.0 \mathrm{~Hz}, 1 \mathrm{H}, 5-\mathrm{H}$ ), 2.31 (ddd, $\left.J=14.1,12.0,2.4 \mathrm{~Hz}, 1 \mathrm{H}, 6-\mathrm{H}_{\beta}\right), 2.04(\mathrm{dt}, J=14.4$, $\left.3.0 \mathrm{~Hz}, 1 \mathrm{H}, 6-\mathrm{H}_{\alpha}\right) \mathrm{ppm} ;{ }^{13} \mathrm{C} \mathrm{NMR}\left(75 \mathrm{MHz}, \mathrm{CDCl}_{3}\right)$ : $\delta=32.9(6-\mathrm{C}), 42.6(5-\mathrm{C}), 52.6\left(\mathrm{NHCOOCH}_{3}\right), 55.5(4-\mathrm{C})$, $64.2\left(\mathrm{OCH}_{2} \mathrm{CH}_{2} \mathrm{O}\right), 64.4\left(\mathrm{OCH}_{2} \mathrm{CH}_{2} \mathrm{O}\right), 70.2\left(2-\mathrm{C}_{\mathrm{Cy}}\right), 71.4$ (1-C), $74.1\left(3-\mathrm{C}_{\mathrm{Cy}}\right), 103.0\left(7-\mathrm{C}_{\mathrm{Ar}}\right), 109.3\left(5-\mathrm{C}_{\mathrm{Ar}}\right), 128.5$ (3$\mathrm{C}_{\mathrm{Bz}}$ and $\left.5-\mathrm{C}_{\mathrm{Bz}}\right), 129.6\left(2-\mathrm{C}_{\mathrm{Bz}}\right.$ and $\left.6-\mathrm{C}_{\mathrm{Bz}}\right), 129.9\left(1-\mathrm{C}_{\mathrm{Bz}}\right)$, $132.1\left(6-\mathrm{C}_{\mathrm{Ar}}\right), 132.9\left(8 \mathrm{a}-\mathrm{C}_{\mathrm{Ar}}\right), 133.3\left(4-\mathrm{C}_{\mathrm{Bz}}\right), 144.0(8-$ $\left.\mathrm{C}_{\mathrm{Ar}}\right), 149.2\left(4 \mathrm{a}-\mathrm{C}_{\mathrm{Ar}}\right), 158.9\left(\mathrm{NHCOOCH}_{3}\right), 165.1(\mathrm{PhCO})$ ppm; IR $(\mathrm{KBr}): \bar{v}=3364,2930,1716,1599,1541,1511$, $1455,1370,1339,1274,1127,1071,1048,887,715 \mathrm{~cm}^{-1}$; $[\alpha]_{\mathrm{D}}^{22}=+63.2^{\circ}\left(c=1, \mathrm{CHCl}_{3}\right)$.

\section{General procedure for acetylation}

A solution of $0.99 \mathrm{~g}(+)-37(2.22 \mathrm{mmol})$ or $1.05 \mathrm{~g}(+)-\mathbf{3 8}$ $(2.22 \mathrm{mmol})$ in $8.28 \mathrm{~cm}^{3}$ acetyl chloride $(9.14 \mathrm{~g}, 0.12 \mathrm{~mol})$ was stirred at $\mathrm{rt}$ for $20-24 \mathrm{~h}$. Then, it was poured into $618 \mathrm{~cm}^{3}$ saturated $\mathrm{NaHCO}_{3}$ solution at $0{ }^{\circ} \mathrm{C}$ and extracted with ethyl acetate $\left(4 \times 110 \mathrm{~cm}^{3}\right)$. The combined organic layer was washed with brine, dried over $\mathrm{Na}_{2} \mathrm{SO}_{4}$ and concentrated in vacuo to give (-)-39 or (-)-40.

(-)-2,3-Diacetoxy-5-(2',3'-dihydrobenzo[b][1,4]dioxin-6'yl)-4-(methoxycarbonylamino)cyclohexyl benzoate [(-)-39, $\mathrm{C}_{27} \mathrm{H}_{29} \mathrm{NO}_{10}$ ] Yield: 81\%; colourless semi-solid; ${ }^{1} \mathrm{H}$ NMR $\left(300 \mathrm{MHz}, \mathrm{CDCl}_{3}\right): \delta=8.07$ (dd, $J=7.2,1.2 \mathrm{~Hz}, 2 \mathrm{H}, 2-$ $\mathrm{H}_{\mathrm{Bz}}$ and $\left.6-\mathrm{H}_{\mathrm{Bz}}\right), 7.62\left(\mathrm{t}, J=7.2 \mathrm{~Hz}, 1 \mathrm{H}, 4-\mathrm{H}_{\mathrm{Bz}}\right), 7.49(\mathrm{t}$, $J=7.5 \mathrm{~Hz}, 2 \mathrm{H}, 3-\mathrm{H}_{\mathrm{Bz}}$ and $\left.5-\mathrm{H}_{\mathrm{Bz}}\right), 6.79(\mathrm{~d}, J=8.1 \mathrm{~Hz}, 1 \mathrm{H}$, $\left.8-\mathrm{H}_{\mathrm{Ar}}\right), 6.73\left(\mathrm{~d}, J=1.8 \mathrm{~Hz}, 1 \mathrm{H}, 5-\mathrm{H}_{\mathrm{Ar}}\right), 6.70(\mathrm{dd}, J=8.4$, $\left.1.8 \mathrm{~Hz}, 1 \mathrm{H}, 7-\mathrm{H}_{\mathrm{Ar}}\right), 5.49\left(\mathrm{t}, J=3.0 \mathrm{~Hz}, 1 \mathrm{H}, 1-\mathrm{H}_{\mathrm{Cy}}\right.$ or $2-$ $\left.\mathrm{H}_{\mathrm{Cy}}\right), 5.37\left(\mathrm{dd}, J=10.8,3.0 \mathrm{~Hz}, 1 \mathrm{H}, 3-\mathrm{H}_{\mathrm{Cy}}\right), 5.28(\mathrm{q}$, $J=3.3 \mathrm{~Hz}, 1 \mathrm{H}, 1-\mathrm{H}_{\mathrm{Cy}}$ or $\left.2-\mathrm{H}_{\mathrm{Cy}}\right), 4.44(\mathrm{~d}, J=9.0 \mathrm{~Hz}, 1 \mathrm{H}$, $\mathrm{NH}), 4.27-4.16\left(\mathrm{~m}, 5 \mathrm{H}, \mathrm{OCH}_{2} \mathrm{CH}_{2} \mathrm{O}\right.$ and $\left.4-\mathrm{H}\right), 3.50(\mathrm{~s}, 3 \mathrm{H}$, $\left.\mathrm{NHCOOCH}_{3}\right), 2.95(\mathrm{td}, J=10.8,7.2 \mathrm{~Hz}, 1 \mathrm{H}, 5-\mathrm{H}), 2.23$ (s, 3H, $\left.\mathrm{COCH}_{3}\right), 2.19-2.09\left(\mathrm{~m}, 2 \mathrm{H}, 6-\mathrm{H}_{\alpha}\right.$ and $\left.6-\mathrm{H}_{\beta}\right), 2.02$ (s, 3H, $\left.\mathrm{COCH}_{3}\right)$ ppm; ${ }^{13} \mathrm{C} \mathrm{NMR}\left(75 \mathrm{MHz}, \mathrm{CDCl}_{3}\right)$ : $\delta=20.8\left(\mathrm{COCH}_{3}\right), 21.0\left(\mathrm{COCH}_{3}\right), 33.6$ (6-C), 42.9 (5-C), $52.1\left(\mathrm{NHCOOCH}_{3}\right), 53.4(4-\mathrm{C}), 64.3\left(\mathrm{OCH}_{2} \mathrm{CH}_{2} \mathrm{O}\right), 69.3$
$\left(2-\mathrm{C}_{\mathrm{Cy}}\right), 69.4$ (1-C), $71.4\left(3-\mathrm{C}_{\mathrm{Cy}}\right), 116.5\left(5-\mathrm{C}_{\mathrm{Ar}}\right), 117.3$ (8$\left.\mathrm{C}_{\mathrm{Ar}}\right), 120.6\left(7-\mathrm{C}_{\mathrm{Ar}}\right), 128.6\left(3-\mathrm{C}_{\mathrm{Bz}}\right.$ and $\left.5-\mathrm{C}_{\mathrm{Bz}}\right), 129.4$ (1$\left.\mathrm{C}_{\mathrm{Bz}}\right), 129.8\left(2-\mathrm{C}_{\mathrm{Bz}}\right.$ and $\left.6-\mathrm{C}_{\mathrm{Bz}}\right), 133.5\left(4-\mathrm{C}_{\mathrm{Bz}}\right), 133.6$ (6$\left.\mathrm{C}_{\mathrm{Ar}}\right), 142.6\left(8 \mathrm{a}-\mathrm{C}_{\mathrm{Ar}}\right), 143.4\left(4 \mathrm{a}-\mathrm{C}_{\mathrm{Ar}}\right), 156.4\left(\mathrm{NHCOOCH}_{3}\right)$, 164.9 (PhCO), $169.4\left(\mathrm{COCH}_{3}\right), 170.7\left(\mathrm{COCH}_{3}\right) \mathrm{ppm}$; IR $(\mathrm{KBr}): \bar{v}=3397,2926,1749,1721,1591,1522,1509$, 1456, 1373, 1271, 1249, 1157, 1070, 1052, 933, 887, 808, $776,717 \mathrm{~cm}^{-1} ;[\alpha]_{\mathrm{D}}^{22}=-22.4^{\circ}\left(c=1, \mathrm{CHCl}_{3}\right)$.

\section{(-)-2,3-Diacetoxy-5-(8'-methoxy-2', 3'-dihy-}

drobenzo[b][1,4]dioxin-6'-yl)-4-(methoxycarbonylamino)cyclohexyl benzoate $\left[(-)-40, \mathrm{C}_{28} \mathrm{H}_{31} \mathrm{NO}_{11}\right]$ Yield: $100 \%$; white solid (fluffy); m.p.: $114-116{ }^{\circ} \mathrm{C} ;{ }^{1} \mathrm{H} \quad \mathrm{NMR}$ $\left(300 \mathrm{MHz}, \mathrm{CDCl}_{3}\right): \delta=8.08(\mathrm{dd}, J=7.2,1.2 \mathrm{~Hz}, 2 \mathrm{H}, 2-$ $\mathrm{H}_{\mathrm{Bz}}$ and $\left.6-\mathrm{H}_{\mathrm{Bz}}\right), 7.62\left(\mathrm{t}, J=7.5 \mathrm{~Hz}, 1 \mathrm{H}, 4-\mathrm{H}_{\mathrm{Bz}}\right), 7.49(\mathrm{t}$, $J=7.5 \mathrm{~Hz}, 2 \mathrm{H}, 3-\mathrm{H}_{\mathrm{Bz}}$ and $\left.5-\mathrm{H}_{\mathrm{Bz}}\right), 6.40(\mathrm{~d}, J=1.8 \mathrm{~Hz}, 1 \mathrm{H}$, $5-\mathrm{H}_{\mathrm{Ar}}$ or $\left.7-\mathrm{H}_{\mathrm{Ar}}\right), 6.28\left(\mathrm{~s}, 1 \mathrm{H}, 5-\mathrm{H}_{\mathrm{Ar}}\right.$ or $\left.7-\mathrm{H}_{\mathrm{Ar}}\right), 5.49(\mathrm{t}$, $J=3.3 \mathrm{~Hz}, 1 \mathrm{H}, 1-\mathrm{H}_{\mathrm{Cy}}$ or $\left.2-\mathrm{H}_{\mathrm{Cy}}\right), 5.38(\mathrm{dd}, J=10.5$, $\left.3.0 \mathrm{~Hz}, 1 \mathrm{H}, 3-\mathrm{H}_{\mathrm{Cy}}\right), 5.28\left(\mathrm{q}, J=3.3 \mathrm{~Hz}, 1 \mathrm{H}, 1-\mathrm{H}_{\mathrm{Cy}}\right.$ or $2-$ $\left.\mathrm{H}_{\mathrm{Cy}}\right), 4.49(\mathrm{~d}, J=9.9 \mathrm{~Hz}, 1 \mathrm{H}, \mathrm{NH}), 4.29-4.21(\mathrm{~m}, 5 \mathrm{H}$, $\left.\mathrm{OCH}_{2} \mathrm{CH}_{2} \mathrm{O}, 4-\mathrm{H}\right), 3.86\left(\mathrm{~s}, 3 \mathrm{H}, \mathrm{OCH}_{3}\right), 3.51(\mathrm{~s}, 3 \mathrm{H}$, $\left.\mathrm{NHCOOCH}_{3}\right), 2.96(\mathrm{td}, J=11.1,7.2 \mathrm{~Hz}, 1 \mathrm{H}, 5-\mathrm{H}), 2.23$ $\left(\mathrm{s}, 3 \mathrm{H}, \mathrm{COCH}_{3}\right), 2.19\left(\mathrm{dd}, J=8.4,2.4 \mathrm{~Hz}, 1 \mathrm{H}, 6-\mathrm{H}_{\beta}\right), 2.12$ (dt, $\left.J=15.3,2.7 \mathrm{~Hz}, 1 \mathrm{H}, 6-\mathrm{H}_{\alpha}\right), 2.02\left(\mathrm{~s}, 3 \mathrm{H}, \mathrm{COCH}_{3}\right)$ ppm; ${ }^{13} \mathrm{C}$ NMR $\left(75 \mathrm{MHz}, \mathrm{CDCl}_{3}\right): \delta=20.8\left(\mathrm{COCH}_{3}\right)$, $21.0\left(\mathrm{COCH}_{3}\right), 33.9(6-\mathrm{C}), 43.2(5-\mathrm{C}), 52.1\left(\mathrm{NHCOOCH}_{3}\right)$, 53.5 (4-C), $64.2\left(\mathrm{OCH}_{2} \mathrm{CH}_{2} \mathrm{O}\right), 64.5\left(\mathrm{OCH}_{2} \mathrm{CH}_{2} \mathrm{O}\right), 69.3$ $\left(2-\mathrm{C}_{\mathrm{Cy}}\right), 69.4(1-\mathrm{C}), 71.4\left(3-\mathrm{C}_{\mathrm{Cy}}\right), 103.7\left(7-\mathrm{C}_{\mathrm{Ar}}\right), 109.1$ (5$\left.\mathrm{C}_{\mathrm{Ar}}\right), 128.6\left(3-\mathrm{C}_{\mathrm{Bz}}\right.$ and $\left.5-\mathrm{C}_{\mathrm{Bz}}\right), 129.4\left(1-\mathrm{C}_{\mathrm{Bz}}\right), 129.8\left(2-\mathrm{C}_{\mathrm{Bz}}\right.$ and 6- $\left.\mathrm{C}_{\mathrm{BZ}}\right), 133.6\left(6-\mathrm{C}_{\mathrm{Ar}}\right), 120.6\left(8 \mathrm{a}-\mathrm{C}_{\mathrm{Ar}}\right), 133.5\left(4-\mathrm{C}_{\mathrm{Bz}}\right)$, $143.9\left(8-\mathrm{C}_{\mathrm{Ar}}\right), 148.8\left(4 \mathrm{a}-\mathrm{C}_{\mathrm{Ar}}\right), 156.5\left(\mathrm{NHCOOCH}_{3}\right), 164.9$ ( $\mathrm{PhCO}), 169.4\left(\mathrm{COCH}_{3}\right), 170.6\left(\mathrm{COCH}_{3}\right) \mathrm{ppm}$; IR $(\mathrm{KBr})$ : $\bar{v}=3365,2930,1750,1726,1599,1540,1512,1455,1370$, $1273,1240,1128,1051,887,715 \mathrm{~cm}^{-1} ;[\alpha]_{\mathrm{D}}^{22}=-6.0^{\circ}$ $\left(c=1, \mathrm{CHCl}_{3}\right)$.

\section{General procedure for the modified Bischler- Napieralski cyclisation}

A solution of $1.39 \mathrm{~g} \mathrm{(-)-39}(2.64 \mathrm{mmol})$ or $1.47 \mathrm{~g} \mathrm{(-)-40}$ (2.64 mmol) and $0.97 \mathrm{~g}$ 4-(dimethylamino)pyridine (7.94 mmol) in $73 \mathrm{~cm}^{3}$ anhydrous dichloromethane was cooled to $0{ }^{\circ} \mathrm{C}$. A solution of $2.34 \mathrm{~cm}^{3}$ trifluoromethanesulphonic anhydride $(3.92 \mathrm{~g}, 13.91 \mathrm{mmol})$ in $12 \mathrm{~cm}^{3}$ anhydrous dichloromethane was added dropwise. The reaction mixture was stirred for $20-24 \mathrm{~h}$ while being allowed to warm to rt. Then, it was diluted with $46 \mathrm{~cm}^{3}$ dichloromethane, subsequently washed with $656 \mathrm{~cm}^{3}$ saturated $\mathrm{NaHCO}_{3}$ solution, $656 \mathrm{~cm}^{3} 20 \%$ aqueous $\mathrm{AcOH}$, and $656 \mathrm{~cm}^{3}$ saturated $\mathrm{NaHCO}_{3}$ solution. The organic layer was dried over $\mathrm{Na}_{2} \mathrm{SO}_{4}$ and evaporated in vacuo. The crude product was purified as specified. 
(-)-2-Benzoyloxy-6-methoxy-1,2,3,4,4a,9,10,12b-octahydro[1,4]dioxino[2,3-j]phenanthridin-3,4-diyl diacetate [(-)41, $\left.\mathrm{C}_{27} \mathrm{H}_{27} \mathrm{NO}_{9}\right]$ This product was converted spontaneously into the corresponding lactam due to traces of acid. It was separated from the lactam derivative by column chromatography $\left(\mathrm{CHCl}_{3}\right.$ /acetone, $\left.20: 1\right)$ to afford a pale yellow oil. Yield: $20 \% ; R_{f}=0.69\left(\mathrm{CH}_{2} \mathrm{Cl}_{2} /\right.$ methanol, 100:1); ${ }^{1} \mathrm{H}$ NMR $\left(300 \mathrm{MHz}, \mathrm{CDCl}_{3}\right): \delta=8.05 \quad$ (dd, $J=8.1,1.2 \mathrm{~Hz}, 2 \mathrm{H}, 2-\mathrm{H}_{\mathrm{Bz}}$ and $\left.6-\mathrm{H}_{\mathrm{Bz}}\right), 7.58(\mathrm{t}, J=7.5 \mathrm{~Hz}$, $\left.1 \mathrm{H}, 4-\mathrm{H}_{\mathrm{Bz}}\right), 7.44\left(\mathrm{t}, J=7.5 \mathrm{~Hz}, 2 \mathrm{H}, 3-\mathrm{H}_{\mathrm{Bz}}\right.$ and $\left.5-\mathrm{H}_{\mathrm{Bz}}\right), 7.21$ $\left(\mathrm{s}, 1 \mathrm{H}, 7-\mathrm{H}_{\mathrm{Ar}}\right), 6.74\left(\mathrm{~s}, 1 \mathrm{H}, 12-\mathrm{H}_{\mathrm{Ar}}\right), 5.57-5.53(\mathrm{~m}, 1 \mathrm{H}$, 3-H), 5.52 (dd, $J=10.8,3.0 \mathrm{~Hz}, 1 \mathrm{H}, 4-\mathrm{H}), 5.43$ (q, $J=2.4 \mathrm{~Hz}, 1 \mathrm{H}, 2-\mathrm{H}), 4.30-4.20\left(\mathrm{~m}, 4 \mathrm{H}, \mathrm{OCH}_{2} \mathrm{CH}_{2} \mathrm{O}\right)$, $3.80\left(\mathrm{~s}, 3 \mathrm{H}, \mathrm{OCH}_{3}\right), 3.51(\mathrm{dd}, J=13.8,10.8 \mathrm{~Hz}, 1 \mathrm{H}, 4 \mathrm{a}-$ $\mathrm{H}), 2.90(\mathrm{td}, J=12.9,3.6 \mathrm{~Hz}, 1 \mathrm{H}, 12 \mathrm{~b}-\mathrm{H}), 2.60(\mathrm{dt}$, $\left.J=14.4,3.0 \mathrm{~Hz}, 1 \mathrm{H}, 1-\mathrm{H}_{\alpha}\right), 2.13\left(\mathrm{~s}, 3 \mathrm{H}, \mathrm{COCH}_{3}\right), 2.09$ (s, $\left.3 \mathrm{H}, \mathrm{COCH}_{3}\right), 2.07-1.97\left(\mathrm{~m}, 1 \mathrm{H}, 1-\mathrm{H}_{\beta}\right) \mathrm{ppm} ;{ }^{13} \mathrm{C} \mathrm{NMR}$ $\left(75 \mathrm{MHz}, \mathrm{CDCl}_{3}\right): \delta=20.8\left(\mathrm{COCH}_{3}\right), 21.0\left(\mathrm{COCH}_{3}\right), 27.4$ (1-C), 32.8 (12b-C), $52.5\left(\mathrm{NCOCH}_{3}\right), 57.5$ (4a-C), 64.2 $\left(\mathrm{OCH}_{2} \mathrm{CH}_{2} \mathrm{O}\right), 64.6\left(\mathrm{OCH}_{2} \mathrm{CH}_{2} \mathrm{O}\right), 69.4$ (3-C), 69.6 (2-C), 72.4 (4-C), $112.5\left(12-\mathrm{C}_{\mathrm{Ar}}\right), 114.5\left(7-\mathrm{C}_{\mathrm{Ar}}\right), 119.1\left(6 \mathrm{a}-\mathrm{C}_{\mathrm{Ar}}\right)$, $128.5\left(3-\mathrm{C}_{\mathrm{Bz}}\right.$ and 5-C $\left.\mathrm{C}_{\mathrm{Bz}}\right), 129.4\left(1-\mathrm{C}_{\mathrm{Bz}}\right), 129.8\left(3-\mathrm{C}_{\mathrm{Bz}}\right.$ and 5- $\left.\mathrm{C}_{\mathrm{Bz}}\right), 133.5\left(4-\mathrm{C}_{\mathrm{Bz}}\right), 134.4\left(12 \mathrm{a}-\mathrm{C}_{\mathrm{Ar}}\right), 142.1\left(8 \mathrm{a}-\mathrm{C}_{\mathrm{Ar}}\right)$, $146.1 \quad\left(4 \mathrm{a}-\mathrm{C}_{\mathrm{Ar}}\right), 160.5$ (6-C), $165.1 \quad$ (PhCO), 169.4 $\left(\mathrm{COCH}_{3}\right), 170.5\left(\mathrm{COCH}_{3}\right) \mathrm{ppm} ;[\alpha]_{\mathrm{D}}^{22}=-63.9^{\circ}(c=1$, $\mathrm{CHCl}_{3}$ ).

\section{(-)-2-Benzoyloxy-6,7-dimethoxy-1,2,3,4,4a,9,10,12b-oc-}

tahydro[1,4]dioxino[2,3-j]phenanthridin-3,4-diyl diacetate $\left[(-)-42, \quad \mathrm{C}_{28} \mathrm{H}_{29} \mathrm{NO}_{10}\right] /(-)-2-B e n z o y l o x y-6,11$-dimethoxy$1,2,3,4,4 a, 8,9,12 b-o c t a h y d r o[1,4]$ dioxino[2,3-i]phenan-

thridin-3,4-diyl diacetate [(-)-43, $\left.\mathrm{C}_{28} \mathrm{H}_{29} \mathrm{NO}_{10}\right]$ It was purified by column chromatography $\left(\mathrm{CHCl}_{3} /\right.$ acetone, $\left.20: 1\right)$ to give a light brown solid (fluffy). Yield: $87 \%$; mixture of regioisomers (1:1); $R_{f}=0.53\left(\mathrm{CH}_{2} \mathrm{Cl}_{2} /\right.$ methanol, $\left.20: 1\right) ;{ }^{1} \mathrm{H}$ NMR $\left(300 \mathrm{MHz}, \mathrm{CDCl}_{3}\right): \delta=8.07(\mathrm{dd}, J=6.9,1.5 \mathrm{~Hz}$, $2 \mathrm{H}, 2-\mathrm{H}_{\mathrm{Bz}}$ and $\left.6-\mathrm{H}_{\mathrm{Bz}}\right), 8.05\left(\mathrm{dd}, J=6.9,1.5 \mathrm{~Hz}, 2 \mathrm{H}, 2-\mathrm{H}_{\mathrm{Bz}}\right.$ and $\left.6-\mathrm{H}_{\mathrm{Bz}}\right), 7.58\left(\mathrm{tt}, J=7.5,1.5 \mathrm{~Hz}, 1 \mathrm{H}, 4-\mathrm{H}_{\mathrm{Bz}}\right), 7.46(\mathrm{t}$, $J=7.5 \mathrm{~Hz}, 2 \mathrm{H}, 3-\mathrm{H}_{\mathrm{Bz}}$ and $\left.5-\mathrm{H}_{\mathrm{Bz}}\right), 7.45(\mathrm{t}, J=7.5 \mathrm{~Hz}, 2 \mathrm{H}$, $3-\mathrm{H}_{\mathrm{Bz}}$ and $\left.5-\mathrm{H}_{\mathrm{Bz}}\right), 6.55\left(\mathrm{~s}, 1 \mathrm{H}, \mathrm{H}_{\mathrm{Ar}}\right), 6.40\left(\mathrm{~s}, 1 \mathrm{H}, \mathrm{H}_{\mathrm{Ar}}\right)$, 5.57-5.53 (m, 2H, $2 \times 3-\mathrm{H}), 5.49(\mathrm{dd}, J=10.8,3.0 \mathrm{~Hz}$, $2 \mathrm{H}, 2 \times 4-\mathrm{H}), 5.46-5.40(\mathrm{~m}, 2 \mathrm{H}, 2 \times 2-\mathrm{H}), 4.42-4.16(\mathrm{~m}$, $\left.8 \mathrm{H}, 2 \times \mathrm{OCH}_{2} \mathrm{CH}_{2} \mathrm{O}\right), 3.91\left(\mathrm{~s}, 3 \mathrm{H}, \mathrm{Ar}-\mathrm{OCH}_{3}\right), 3.84-3.83$ $\left(3 \times \mathrm{s}, \quad 9 \mathrm{H}, \quad \mathrm{Ar}-\mathrm{OCH}_{3}, 2 \times \mathrm{CNOCH}_{3}\right), 3.40(2 \times \mathrm{dd}$, $J=13.5,10.5 \mathrm{~Hz}, 2 \mathrm{H}, 2 \times 4 \mathrm{a}-\mathrm{H}), 2.82-2.55(\mathrm{~m}, 4 \mathrm{H}$, $\left.2 \times 12 \mathrm{~b}-\mathrm{H}, 2 \times 1-\mathrm{H}_{\alpha}\right), 2.12\left(\mathrm{~s}, 6 \mathrm{H}, 2 \times \mathrm{COCH}_{3}\right), 2.09(\mathrm{~s}$, $\left.3 \mathrm{H}, \mathrm{COCH}_{3}\right), 2.08\left(\mathrm{~s}, 3 \mathrm{H}, \mathrm{COCH}_{3}\right), 1.90(2 \times \mathrm{dt}, J=12.3$, $\left.2.7 \mathrm{~Hz}, 2 \mathrm{H}, 1-\mathrm{H}_{\beta}\right) \mathrm{ppm} ;{ }^{13} \mathrm{C} \mathrm{NMR}\left(75 \mathrm{MHz}, \mathrm{CDCl}_{3}\right)$ : $\delta=20.8\left(\mathrm{COCH}_{3}\right), 21.0\left(\mathrm{COCH}_{3}\right), 27.5$ (1-C), 27.6 (1-C), 33.4 (10b-C), 33.6 (10b-C), $52.6\left(2 \times \mathrm{CNOCH}_{3}\right), 56.9$ $\left(\mathrm{OCH}_{3}\right), 57.0(2 \times 4 \mathrm{a}-\mathrm{C}), 61.7\left(\mathrm{OCH}_{3}\right), 63.9\left(\mathrm{OCH}_{2} \mathrm{CH}_{2-}\right.$ $\mathrm{O}), \quad 64.2\left(\mathrm{OCH}_{2} \mathrm{CH}_{2} \mathrm{O}\right), \quad 64.3 \quad\left(\mathrm{OCH}_{2} \mathrm{CH}_{2} \mathrm{O}\right), \quad 64.5$
$\left(\mathrm{OCH}_{2} \mathrm{CH}_{2} \mathrm{O}\right), 69.3\left(2 \times 3-\mathrm{C}_{\mathrm{Cy}}\right), 69.6\left(2-\mathrm{C}_{\mathrm{Cy}}\right), 69.7$ $\mathrm{C}_{\mathrm{Cy}}$ ), 72.4 (4-C), 72.6 (4-C), 99.4 (12-C), 108.1 (12-C), $128.5\left(3-\mathrm{C}_{\mathrm{Bz}}\right.$ and $\left.5-\mathrm{C}_{\mathrm{Bz}}\right), 128.6\left(3-\mathrm{C}_{\mathrm{Bz}}\right.$ and $\left.5-\mathrm{C}_{\mathrm{Bz}}\right), 129.3$ $\left(1-\mathrm{C}_{\mathrm{Bz}}\right), 129.4\left(1-\mathrm{C}_{\mathrm{Bz}}\right), 129.8\left(2-\mathrm{C}_{\mathrm{Bz}}\right.$ and $\left.6-\mathrm{C}_{\mathrm{Bz}}\right), 132.1$ $(2 \times 6 \mathrm{a}-\mathrm{C}), 133.4\left(4-\mathrm{C}_{\mathrm{Bz}}\right), 133.5\left(4-\mathrm{C}_{\mathrm{Bz}}\right), 134.9(2 \times 12 \mathrm{a}-$ C), 135.3 (7a-C and 10a-C), 142.5 (6b-C), 146.5 (7-C), 150.6 (11-C and 11a-C), 160.4 (6-C), 165.1 (PhCO), 165.2 $(\mathrm{PhCO}), 169.4\left(2 \times \mathrm{COCH}_{3}\right), 170.4\left(2 \times \mathrm{COCH}_{3}\right) \mathrm{ppm}$; IR $(\mathrm{KBr}): \bar{v}=2944,1752,1637,1600,1500,1437,1371$, $1334,1269,1239,1096,1070,714 \mathrm{~cm}^{-1} ;[\alpha]_{\mathrm{D}}^{22}=-114.1^{\circ}$ $\left(c=1, \mathrm{CHCl}_{3}\right)$.

\section{General procedure for the synthesis of lactams $(-)-44$ and $( \pm)-45 /( \pm)-46$}

To a solution of $0.61 \mathrm{~g}(-)-41(1.13 \mathrm{mmol})$ or $0.70 \mathrm{~g}( \pm)$ 42/(土)-43 (1.13 mmol) in $55 \mathrm{~cm}^{3}$ tetrahydrofuran, $2.80 \mathrm{~cm}^{3} 2 \mathrm{M}$ aqueous $\mathrm{HCl}$ was added, and it was stirred at $\mathrm{rt}$ for $22 \mathrm{~h}$. Then, it was poured into $120 \mathrm{~cm}^{3}$ saturated $\mathrm{NaHCO}_{3}$ solution and extracted with ethyl acetate $\left(4 \times 30 \mathrm{~cm}^{3}\right)$. The combined organic layer was washed with brine, dried over $\mathrm{Na}_{2} \mathrm{SO}_{4}$ and the solvent was evaporated in vacuo. The crude product was dissolved in $5.30 \mathrm{~cm}^{3}$ acetyl chloride $(4.78 \mathrm{~g}, 0.061 \mathrm{~mol})$ and stirred at rt for $20 \mathrm{~h}$. Then, it was poured into $380 \mathrm{~cm}^{3}$ saturated $\mathrm{NaHCO}_{3}$ solution and extracted with ethyl acetate $\left(4 \times 80 \mathrm{~cm}^{3}\right)$. The combined organic layer was washed with brine, dried over $\mathrm{Na}_{2} \mathrm{SO}_{4}$, and the solvent was evaporated in vacuo to afford $(-)-44$ or $( \pm)-\mathbf{4 5} /( \pm)-\mathbf{4 6}$.

(-)-2-Benzoyloxy-6-oxo-1,2,3,4,4a,9,10,12b-octahydro[1,4]dioxino[2,3-j]phenanthridine-3,4-diyl diacetate [(-)-44, $\mathrm{C}_{26} \mathrm{H}_{25} \mathrm{NO}_{9}$ ] Yield: 54\%; yellow oil; ${ }^{1} \mathrm{H}$ NMR (300 MHz, $\left.\mathrm{CDCl}_{3}\right): \delta=8.05\left(\mathrm{dd}, J=7.2,1.2 \mathrm{~Hz}, 2 \mathrm{H}, 2-\mathrm{H}_{\mathrm{Bz}}\right.$ and 6$\left.\mathrm{H}_{\mathrm{Bz}}\right), 7.59\left(\mathrm{tt}, J=7.5,1.2 \mathrm{~Hz}, 1 \mathrm{H}, 4-\mathrm{H}_{\mathrm{Bz}}\right), 7.46(\mathrm{t}$, $J=7.5 \mathrm{~Hz}, 2 \mathrm{H}, 3-\mathrm{H}_{\mathrm{Bz}}$ and $\left.5-\mathrm{H}_{\mathrm{Bz}}\right), 6.74\left(\mathrm{~s}, 1 \mathrm{H}, 7-\mathrm{H}_{\mathrm{Ar}}\right)$, $6.57\left(\mathrm{~s}, 1 \mathrm{H}, 12-\mathrm{H}_{\mathrm{Ar}}\right), 6.08(\mathrm{~s}, 1 \mathrm{H}, \mathrm{NH}), 5.60(\mathrm{t}, J=3.0 \mathrm{~Hz}$, $1 \mathrm{H}, 3-\mathrm{H}), 5.44$ (q, $J=3.0 \mathrm{~Hz}, 1 \mathrm{H}, 2-\mathrm{H}), 5.36(\mathrm{dd}, J=10.8$, $3.0 \mathrm{~Hz}, 1 \mathrm{H}, 4-\mathrm{H}), 4.31-4.22\left(\mathrm{~m}, 4 \mathrm{H}, \mathrm{OCH}_{2} \mathrm{CH}_{2} \mathrm{O}\right), 3.87$ $(\mathrm{dd}, J=12.0,11.1 \mathrm{~Hz}, 1 \mathrm{H}, 4 \mathrm{a}-\mathrm{H}), 3.26(\mathrm{td}, J=12.6$, $3.6 \mathrm{~Hz}, 1 \mathrm{H}, 12 \mathrm{~b}-\mathrm{H}), 2.62\left(\mathrm{dt}, J=14.4,3.0 \mathrm{~Hz}, 1 \mathrm{H}, 1-\mathrm{H}_{\alpha}\right)$, $2.11\left(\mathrm{~s}, 3 \mathrm{H}, \mathrm{COCH}_{3}\right), 2.10\left(\mathrm{~s}, 3 \mathrm{H}, \mathrm{COCH}_{3}\right), 2.08-1.08(\mathrm{~m}$, $\left.1 \mathrm{H}, 1-\mathrm{H}_{\beta}\right) \mathrm{ppm} ;{ }^{13} \mathrm{C} \mathrm{NMR}\left(75 \mathrm{MHz}, \mathrm{CDCl}_{3}\right): \delta=20.8$ $\left(\mathrm{COCH}_{3}\right), 20.9\left(\mathrm{COCH}_{3}\right), 26.8$ (1-C), 34.7 (12b-C), 53.0 (4a-C), $64.1\left(\mathrm{OCH}_{2} \mathrm{CH}_{2} \mathrm{O}\right), 64.8\left(\mathrm{OCH}_{2} \mathrm{CH}_{2} \mathrm{O}\right), 67.7$ (2-C), 69.3 (4-C), 71.9 (3-C), 112.6 (7-C), 117.8 (12-C), 122.3 $(6 \mathrm{a}-\mathrm{C}), 128.7\left(3-\mathrm{C}_{\mathrm{Bz}}, 5-\mathrm{C}_{\mathrm{Bz}}\right), 129.1\left(1-\mathrm{C}_{\mathrm{Bz}}\right), 129.8\left(2-\mathrm{C}_{\mathrm{Bz}}\right.$, 6- $\mathrm{C}_{\mathrm{Bz}}$ ), $133.7\left(4-\mathrm{C}_{\mathrm{Bz}}\right), 137.0(12 \mathrm{a}-\mathrm{C}), 142.8$ (7a-C), 147.4 (11a-C), 165.0 (6-C), 165.7 (PhCO), $169.2\left(\mathrm{COCH}_{3}\right), 170.3$ $\left(\mathrm{COCH}_{3}\right)$ ppm; IR $(\mathrm{KBr}): \bar{v}=2928,1754,1726,1669$, 1498, 1455, 1368, 1368, 1317, 1266, 1234, 1097, 1065, $925,803,712 \mathrm{~cm}^{-1} ;[\alpha]_{\mathrm{D}}^{22}=-78.7^{\circ}\left(c=1, \mathrm{CHCl}_{3}\right)$. 
(士)-2-Benzoyloxy-7-methoxy-6-oxo-1,2,3,4,4a,8,9,12b-octahydro[1,4]dioxino[2,3-j]phenanthridine-3,4-diyl diacetate $\left[( \pm)-45, \quad \mathrm{C}_{27} \mathrm{H}_{27} \mathrm{NO}_{10}\right] /( \pm)-2-B e n z o y l o x y-11-m e t h o x y-6-0 x 0-$ $1,2,3,4,4 a, 8,9,12 b$-octahydro[1,4]dioxino[2,3-i]phenan-

thridine-3,4-diyl diacetate $\left[( \pm)-46, \mathrm{C}_{27} \mathrm{H}_{27} \mathrm{NO}_{10}\right]$ The mixture of these regioisomers $(1: 1)$ proved to be inseparable in this step. Their isolation was achieved after selective demethylation, as described below.

\section{General procedure for the Zemplén deacylation}

$77 \mathrm{~cm}^{3} 0.56 \mathrm{M}$ methanolic solution of sodium methoxide was added dropwise at $\mathrm{rt}$ to a solution of $1.15 \mathrm{~g}(-)-42 /$ $(-)-43(2.13 \mathrm{mmol})$ in $153 \mathrm{~cm}^{3}$ anhydrous tetrahydrofuran, and the reaction mixture was stirred for $2 \mathrm{~h}$. Then it was poured into $500 \mathrm{~cm}^{3}$ water and extracted with ethyl acetate $\left(4 \times 120 \mathrm{~cm}^{3}\right)$. The combined organic layer was washed with brine, dried over $\mathrm{Na}_{2} \mathrm{SO}_{4}$, and the solvent was evaporated in vacuo. The regioisomers were separated by column chromatography (EtOAc/ethanol, 20:1) to give $(-)-50$ and (-)-51.

(-)-6,7-Dimethoxy-1,2,3,4,4a,9,10,12b-octahydro[1,4]dioxino[2,3-j]phenanthridine-2,3,4-triol [(-)-50, $\left.\mathrm{C}_{17} \mathrm{H}_{21} \mathrm{NO}_{7}\right]$ Yield: 22\%; white solid; m.p.: $133-136{ }^{\circ} \mathrm{C} ; R_{f}=0.42$ (EtOAc/methanol, 20:1); ${ }^{1} \mathrm{H}$ NMR (300 MHz, DMSO- $d_{6}$ ): $\delta=6.55(\mathrm{~s}, 1 \mathrm{H}, 12-\mathrm{H}), 4.83(\mathrm{~d}, J=3.3 \mathrm{~Hz}, 1 \mathrm{H}, \mathrm{OH}), 4.53$ $(\mathrm{d}, J=3.6 \mathrm{~Hz}, 1 \mathrm{H}, \mathrm{OH}), 4.31-4.18\left(\mathrm{~m}, 5 \mathrm{H}, \mathrm{OCH}_{2} \mathrm{CH}_{2} \mathrm{O}\right.$ and $\mathrm{OH}), 3.87(\mathrm{q}, J=2.7 \mathrm{~Hz}, 1 \mathrm{H}, 2-\mathrm{H}), 3.79-3.74(\mathrm{~m}, 4 \mathrm{H}$, $3-\mathrm{H}$ and $\left.\mathrm{OCH}_{3}\right), 3.71\left(\mathrm{~s}, 3 \mathrm{H}, \mathrm{CNOCH}_{3}\right), 3.68(\mathrm{dd}, J=10.2$, $3.0 \mathrm{~Hz}, 1 \mathrm{H}, 4-\mathrm{H}), 2.87$ (dd, $J=13.2,9.9 \mathrm{~Hz}, 1-\mathrm{H}, 4 \mathrm{a}-\mathrm{H})$, 2.39 (td, $J=12.9,3.3 \mathrm{~Hz}, 1 \mathrm{H}, 12 \mathrm{~b}-\mathrm{H}), 2.04$ (dt, $J=13.8$, $3.3 \mathrm{~Hz}, 1 \mathrm{H}, 1-\mathrm{H}_{\alpha}$ ), 1.62 (ddd, $J=14.4,11.1,1.8 \mathrm{~Hz}, 1 \mathrm{H}$, $\left.1-\mathrm{H}_{\beta}\right) \mathrm{ppm} ;{ }^{13} \mathrm{C}$ NMR $\left(75 \mathrm{MHz}, \mathrm{DMSO}-d_{6}\right): \delta=28.9(1-$ C), 32.2 (12b-C), $52.1\left(\mathrm{CNOCH}_{3}\right), 58.9$ (4a-C), 60.8 $\left(\mathrm{OCH}_{3}\right), 63.7\left(\mathrm{OCH}_{2} \mathrm{CH}_{2} \mathrm{O}\right), 64.2\left(\mathrm{OCH}_{2} \mathrm{CH}_{2} \mathrm{O}\right), 68.6$ (2C), 71.2 (4-C), 71.8 (3-C), 107.5 (12-C), 111.9 (6a-C), 135.8 (7a-C), 136.7 (12a-C), 146.1 (7-C), 146.6 (11a-C), 159.1 (6-C) ppm; IR (KBr): $\bar{v}=3408,2926,1717,1637$, 1608, 1574, 1483, 1437, 1333, 1226, 1122, 1040, $812 \mathrm{~cm}^{-1} ;[\alpha]_{\mathrm{D}}^{22}=-49.0^{\circ}(c=0.3$, methanol $)$.

(-)-6,11-Dimethoxy-1,2,3,4,4a,9,10,12b-octahydro[1,4]dioxino[2,3-i]phenanthridine-2,3,4-triol [(-)-51, $\left.\mathrm{C}_{17} \mathrm{H}_{21} \mathrm{NO}_{7}\right]$ Yield: $23 \%$; white solid; m.p.: $132-134{ }^{\circ} \mathrm{C} ; R_{f}=0.26$ (EtOAc/methanol, 20:1); ${ }^{1} \mathrm{H}$ NMR (300 MHz, DMSO- $d_{6}$ ): $\delta=6.49(\mathrm{~s}, 1 \mathrm{H}, 12-\mathrm{H}), 4.84(\mathrm{~d}, J=3.3 \mathrm{~Hz}, 1 \mathrm{H}, \mathrm{OH}), 4.54$ $(\mathrm{d}, J=3.3 \mathrm{~Hz}, 1 \mathrm{H}, \mathrm{OH}), 4.34-4.14\left(\mathrm{~m}, 5 \mathrm{H}, \mathrm{OCH}_{2} \mathrm{CH}_{2} \mathrm{O}\right.$ and $\mathrm{OH}), 3.89(\mathrm{q}, J=2.4 \mathrm{~Hz}, 1 \mathrm{H}, 2-\mathrm{H}), 3.80$ (s, 3H, $\left.\mathrm{OCH}_{3}\right), 3.77(\mathrm{t}, J=3.0 \mathrm{~Hz}, 1 \mathrm{H}, 3-\mathrm{H}), 3.71(\mathrm{~s}, 3 \mathrm{H}$, $\mathrm{CNOCH}_{3}$ ), 3.69 (overlapped dd, 1H, 4-H), 2.87 (dd, $J=13.8,10.2 \mathrm{~Hz}, 1-\mathrm{H}, 4 \mathrm{a}-\mathrm{H}), 2.42(\mathrm{td}, J=12.9,3.3 \mathrm{~Hz}$, 1H, 12b-H), 2.14 (dt, $J=12.9,3.0 \mathrm{~Hz}, 1 \mathrm{H}, 1-\mathrm{H}_{\alpha}$ ), 1.62 (ddd, $\left.J=14.4,12.0,2.4 \mathrm{~Hz}, 1 \mathrm{H}, 1-\mathrm{H}_{\beta}\right) \mathrm{ppm} ;{ }^{13} \mathrm{C} \mathrm{NMR}$
(75 MHz, DMSO- $\left.d_{6}\right): \delta=28.9$ (1-C), 32.5 (12b-C), 51.9 $\left(\mathrm{CNOCH}_{3}\right), 55.6\left(\mathrm{OCH}_{3}\right), 58.9(4 \mathrm{a}-\mathrm{C}), 63.0\left(\mathrm{OCH}_{2} \mathrm{CH}_{2} \mathrm{O}\right)$, $63.8\left(\mathrm{OCH}_{2} \mathrm{CH}_{2} \mathrm{O}\right), 68.6$ (2-C), 71.3 (4-C), 71.8 (3-C), 99.6 (12-C), 107.2 (6a-C), 131.4 (10a-C), 136.9 (12a-C), 141.9 (6b-C), 150.5 (11-C), 159.4 (6-C) ppm; IR (KBr): $\bar{v}=3420,2924,1716,1699,1635,1602,1558,1457,1384$, 1334, $1132, \quad 1065 \mathrm{~cm}^{-1} ; \quad[\alpha]_{\mathrm{D}}^{22}=-63.8^{\circ} \quad(c=0.3$, methanol).

\section{General procedure for the synthesis of triacetoxy lactams (-)-52 and (-)-53}

To a solution of $0.23 \mathrm{~g}(-)-\mathbf{5 0}(0.65 \mathrm{mmol})$ or $0.23 \mathrm{~g} \mathrm{(-)}$ $51(0.65 \mathrm{mmol})$ in $32 \mathrm{~cm}^{3}$ tetrahydrofuran, $1.60 \mathrm{~cm}^{3} 2 \mathrm{M}$ aqueous $\mathrm{HCl}$ was added and the reaction mixture was stirred at $\mathrm{rt}$ for $22 \mathrm{~h}$. After evaporation of the solvent in vacuo, the residue was dissolved in $2.44 \mathrm{~cm}^{3}$ acetyl chloride $(2.20 \mathrm{~g}, 28.03 \mathrm{mmol})$ and stirred at $\mathrm{rt}$ for $20 \mathrm{~h}$. Then, it was poured into $186 \mathrm{~cm}^{3}$ saturated $\mathrm{NaHCO}_{3}$ solution at $0{ }^{\circ} \mathrm{C}$ and extracted with ethyl acetate $\left(4 \times 40 \mathrm{~cm}^{3}\right)$. The combined organic layer was washed with brine, dried over $\mathrm{Na}_{2} \mathrm{SO}_{4}$, and the solvent was evaporated in vacuo. The crude product was purified by preparative TLC $\left(\mathrm{CHCl}_{3}\right)$ acetone, 3:1) to afford (-)-52 or (-)-53.

(-)-7-Methoxy-6-oxo-1,2,3,4,4a,9,10,12b-octahydro[1,4]dioxino[2,3-j]phenanthridine-2,3,4-triyl triacetate [(-)-52, $\mathrm{C}_{22} \mathrm{H}_{25} \mathrm{NO}_{10}$ ] Yield: 87\%; white solid; m.p.: $122-125{ }^{\circ} \mathrm{C}$; $R_{f}=0.39\left(\mathrm{CHCl}_{3}\right.$ /acetone, 3:1); ${ }^{1} \mathrm{H}$ NMR $(300 \mathrm{MHz}$, $\left.\mathrm{CDCl}_{3}\right): \delta=6.52(\mathrm{~s}, 1 \mathrm{H}, 12-\mathrm{H}), 6.03(\mathrm{~s}, 1 \mathrm{H}, \mathrm{NH}), 5.42(\mathrm{t}$, $J=3.0 \mathrm{~Hz}, 1 \mathrm{H}, 3-\mathrm{H}), 5.18(\mathrm{q}, J=3.0 \mathrm{~Hz} 1 \mathrm{H}, 2-\mathrm{H}), 5.16$ $(\mathrm{dd}, J=11.1,2.7 \mathrm{~Hz}, 1 \mathrm{H}, 4-\mathrm{H}), 4.36-4.26(\mathrm{~m}, 4 \mathrm{H}$, $\left.\mathrm{OCH}_{2} \mathrm{CH}_{2} \mathrm{O}\right), 3.94\left(\mathrm{~s}, 3 \mathrm{H}, \mathrm{OCH}_{3}\right), 3.71-3.63(\mathrm{~m}, 1 \mathrm{H}, 4 \mathrm{a}-$ $\mathrm{H}), 3.06(\mathrm{td}, J=12.6,3.6 \mathrm{~Hz}, 1 \mathrm{H}, 12 \mathrm{~b}-\mathrm{H}), 2.40(\mathrm{dt}$, $\left.J=14.7,3.0 \mathrm{~Hz}, 1 \mathrm{H}, 1-\mathrm{H}_{\alpha}\right), 2.13\left(\mathrm{~s}, 3 \mathrm{H}, \mathrm{COCH}_{3}\right), 2.07$ (s, $\left.3 \mathrm{H}, \mathrm{COCH}_{3}\right), 2.06\left(\mathrm{~s}, 3 \mathrm{H}, \mathrm{COCH}_{3}\right), 1.87$ (ddd, $J=14.7$, 12.9, $\left.2.4 \mathrm{~Hz}, 1 \mathrm{H}, 1-\mathrm{H}_{\beta}\right) \mathrm{ppm} ;{ }^{13} \mathrm{C} \mathrm{NMR}\left(75 \mathrm{MHz}, \mathrm{CDCl}_{3}\right)$ : $\delta=20.7\left(\mathrm{COCH}_{3}\right), 20.8\left(\mathrm{COCH}_{3}\right), 21.0\left(\mathrm{COCH}_{3}\right), 26.7$ (1C), 35.3 (12b-C), $52.2(4 \mathrm{a}-\mathrm{C}), 61.9\left(\mathrm{OCH}_{3}\right), 64.1\left(\mathrm{OCH}_{2-}\right.$ $\left.\mathrm{CH}_{2} \mathrm{O}\right), 64.6\left(\mathrm{OCH}_{2} \mathrm{CH}_{2} \mathrm{O}\right), 67.4$ (3-C), 68.6 (2-C), 71.6 (4C), 107.9 (12-C), 115.5 (6a-C), 133.8 (7a-C), 137.1 (12aC), 147.4 (7-C), 151.0 (11a-C), 163.6 (6-C), 169.1 $\left(\mathrm{COCH}_{3}\right), 169.4\left(\mathrm{COCH}_{3}\right), 170.4\left(\mathrm{COCH}_{3}\right) \mathrm{ppm}$; IR $(\mathrm{KBr})$ : $\bar{v}=3197,3089,2931,2874,1751,1670,1606,1476,1372$, $1332,1244,1224,1118,1062,1041,859 \mathrm{~cm}^{-1} ;[\alpha]_{\mathrm{D}}^{22}=$ $-77.2^{\circ}\left(c=0.5, \mathrm{CHCl}_{3}\right)$.

(-)-11-Methoxy-6-oxo-1,2,3,4,4a,8,9,12b-octahydro[1,4]dioxino[2,3-i]phenanthridine-2,3,4-triyl triacetate [(-)-53, $\mathrm{C}_{22} \mathrm{H}_{25} \mathrm{NO}_{10}$ ] Yield: $47 \%$; white solid; m.p.: $208-211{ }^{\circ} \mathrm{C}$; $R_{f}=0.17 \quad\left(\mathrm{CHCl}_{3}\right.$ /acetone, 3:1); ${ }^{1} \mathrm{H}$ NMR $(300 \mathrm{MHz}$, DMSO- $\left.d_{6}\right): \delta=7.98(\mathrm{~s}, 1 \mathrm{H}, \mathrm{NH}), 6.48(\mathrm{~s}, 1 \mathrm{H}, 12-\mathrm{H}), 5.23$ $(\mathrm{t}, J=3.0 \mathrm{~Hz}, 1 \mathrm{H}, 3-\mathrm{H}), 5.06(\mathrm{q}, J=2.7 \mathrm{~Hz}, 1 \mathrm{H}, 2-\mathrm{H})$, 4.91 (dd, $J=10.8,2.7 \mathrm{~Hz}, 2 \mathrm{H}, 4-\mathrm{H}), 4.37-4.12(\mathrm{~m}, 4 \mathrm{H}$, 
$\left.\mathrm{OCH}_{2} \mathrm{CH}_{2} \mathrm{O}\right), 3.83\left(\mathrm{~s}, 3 \mathrm{H}, \mathrm{OCH}_{3}\right), 3.53-3.45(\mathrm{~m}, 1 \mathrm{H}, 4 \mathrm{a}-$ $\mathrm{H}), 2.95(\mathrm{td}, J=12.3,3.3 \mathrm{~Hz}, 1 \mathrm{H}, 12 \mathrm{~b}-\mathrm{H}), 2.11(\mathrm{~s}, 3 \mathrm{H}$, $\left.\mathrm{COCH}_{3}\right), 2.04\left(\mathrm{~s}, 3 \mathrm{H}, \mathrm{COCH}_{3}\right), 1.95\left(\mathrm{~s}, 3 \mathrm{H}, \mathrm{COCH}_{3}\right), 1.78$ (ddd, $J=14.4,12.9,2.4 \mathrm{~Hz}, 1 \mathrm{H}, 1-\mathrm{H}_{\beta}$ ) ppm (the sign of 1$\mathrm{H}_{\alpha}$ is covered by that of DMSO- $\left.d_{6}\right) ;{ }^{13} \mathrm{C} \mathrm{NMR}(75 \mathrm{MHz}$, DMSO- $\left.d_{6}\right): \quad \delta=20.4 \quad\left(\mathrm{COCH}_{3}\right), 20.7 \quad\left(\mathrm{COCH}_{3}\right), \quad 20.9$ $\left(\mathrm{COCH}_{3}\right), 26.0$ (1-C), 35.3 (12b-C), 51.6 (4a-C), 55.7 $\left(\mathrm{OCH}_{3}\right), 63.0\left(\mathrm{OCH}_{2} \mathrm{CH}_{2} \mathrm{O}\right), 63.1\left(\mathrm{OCH}_{2} \mathrm{CH}_{2} \mathrm{O}\right), 66.6$ (3C), 68.1 (2-C), 70.5 (4-C), 99.5 (12-C), 111.0 (6a-C), 131.9 (7a-C), 134.1 (12a-C), 144.6 (7-C), 150.6 (11a-C), 162.5 (6-C), $169.1\left(\mathrm{COCH}_{3}\right), 169.3\left(\mathrm{COCH}_{3}\right), 169.9\left(\mathrm{COCH}_{3}\right)$ ppm; IR (KBr): $\bar{v}=3195,3091,2939,1752,1667,1597$, 1494, 1451, 1370, 1330, 1251, 1157, 1128, 1060, 1028, $799 \mathrm{~cm}^{-1} ;[\alpha]_{\mathrm{D}}^{22}=-135.5^{\circ}\left(c=0.5, \mathrm{CHCl}_{3}\right)$.

\section{General procedure for the selective demethylation}

To a solution of $0.18 \mathrm{~g}( \pm)-45 /( \pm)-46(0.35 \mathrm{mmol})$ or $0.16 \mathrm{~g}(-)-52(0.35 \mathrm{mmol})$ and $57.3 \mathrm{mg}$ potassium iodide $(0.35 \mathrm{mmol})$ in $16 \mathrm{~cm}^{3}$ anhydrous acetonitrile, $48.7 \mathrm{mg}$ chlorotrimethylsilane $(0.45 \mathrm{mmol})$ in $3.1 \mathrm{~cm}^{3}$ anhydrous acetonitrile was added. The reaction mixture was heated to $60{ }^{\circ} \mathrm{C}$ and stirred for $4 \mathrm{~h}$. Then, it was cooled to $0{ }^{\circ} \mathrm{C}$ and $26 \mathrm{~cm}^{3}$ water was added dropwise to quench the reaction. After extraction with ethyl acetate $\left(4 \times 26 \mathrm{~cm}^{3}\right)$, the combined organic layer was washed with brine, dried over $\mathrm{Na}_{2} \mathrm{SO}_{4}$, and the solvent was evaporated in vacuo. The residue was purified by preparative TLC (EtOAc/heptane, 1:1) to give $( \pm)-\mathbf{4 7}$ or $(-)-54$. Compound $( \pm)-\mathbf{4 6}$, as well as the by-products $( \pm)-\mathbf{4 8}$ and $( \pm)-\mathbf{4 9}$, was isolated from the crude product obtained by the conversion of $( \pm)-\mathbf{4 5}$ / (土)-46.

\section{( \pm )-2-Benzoyloxy-7-hydroxy-6-oxo-1,2,3,4,4a,9,10,12b-oc-} tahydro[1,4]dioxino[2,3-j]phenanthridine-3,4-diyl diacetate [( \pm -47, $\left.\mathrm{C}_{26} \mathrm{H}_{25} \mathrm{NO}_{10}\right]$ Yield: $20 \%$; white solid; m.p.: 201$204{ }^{\circ} \mathrm{C} ;{ }^{1} \mathrm{H}$ NMR $\left(300 \mathrm{MHz}, \mathrm{CDCl}_{3}\right): \delta=12.52(\mathrm{~s}, 1 \mathrm{H}$, $\mathrm{OH}), 8.03\left(\mathrm{dd}, J=7.8,1.2 \mathrm{~Hz}, 2 \mathrm{H}, 2-\mathrm{H}_{\mathrm{Bz}}\right.$ and $\left.6-\mathrm{H}_{\mathrm{Bz}}\right), 7.59$ $\left(\mathrm{tt}, J=7.5,1.2 \mathrm{~Hz}, 1 \mathrm{H}, 4-\mathrm{H}_{\mathrm{Bz}}\right), 7.46(\mathrm{t}, J=7.5 \mathrm{~Hz}, 2 \mathrm{H}, 3-$ $\mathrm{H}_{\mathrm{Bz}}$ and 5- $\left.\mathrm{H}_{\mathrm{Bz}}\right), 6.27(\mathrm{~s}, 1 \mathrm{H}, 12-\mathrm{H}), 6.10(\mathrm{~s}, 1 \mathrm{H}, \mathrm{NH}), 5.60$ (t, $J=3.0 \mathrm{~Hz}, 1 \mathrm{H}, 3-\mathrm{H}), 5.43$ (q, $J=3.0 \mathrm{~Hz}, 1 \mathrm{H}, 2-\mathrm{H})$, $5.33(\mathrm{dd}, J=10.8,2.7 \mathrm{~Hz}, 1 \mathrm{H}, 4-\mathrm{H}), 4.31$ (s, 4H, $\mathrm{OCH}_{2-}$ $\mathrm{CH}_{2} \mathrm{O}$ ), 3.85 (dd, $\left.J=12.3,11.1 \mathrm{~Hz}, 1 \mathrm{H}, 4 \mathrm{a}-\mathrm{H}\right), 3.20$ (td, $J=12.9,3.3 \mathrm{~Hz}, 1 \mathrm{H}, 12 \mathrm{~b}-\mathrm{H}), 2.60$ (dt, $J=14.7,2.7 \mathrm{~Hz}$, $\left.1 \mathrm{H}, 1-\mathrm{H}_{\alpha}\right), 2.12\left(\mathrm{~s}, 3 \mathrm{H}, \mathrm{COCH}_{3}\right), 2.11\left(\mathrm{~s}, 3 \mathrm{H}, \mathrm{COCH}_{3}\right)$, 2.01 (ddd, $\left.J=14.7,12.6,2.4 \mathrm{~Hz}, 1 \mathrm{H}, 1-\mathrm{H}_{\beta}\right) \mathrm{ppm} ;{ }^{13} \mathrm{C}$ NMR $\quad\left(75 \mathrm{MHz}, \quad \mathrm{CDCl}_{3}\right): \quad \delta=20.7 \quad\left(\mathrm{COCH}_{3}\right), \quad 20.8$ $\left(\mathrm{COCH}_{3}\right), 26.5$ (1-C), 34.3 (12b-C), 52.8 (4a-C), 64.1 $\left(\mathrm{OCH}_{2} \mathrm{CH}_{2} \mathrm{O}\right), 64.8\left(\mathrm{OCH}_{2} \mathrm{CH}_{2} \mathrm{O}\right), 67.5$ (3-C), 69.1 (2-C), 71.9 (4-C), 103.7 (12-C), 104.5 (6a-C), 128.6 (3- $\mathrm{C}_{\mathrm{Bz}}$ and 5$\left.\mathrm{C}_{\mathrm{Bz}}\right), 129.1\left(1-\mathrm{C}_{\mathrm{Bz}}\right), 129.8\left(2-\mathrm{C}_{\mathrm{Bz}}\right.$ and 6- $\left.\mathrm{C}_{\mathrm{Bz}}\right), 131.1(7 \mathrm{a}-\mathrm{C})$,
132.2 (12a-C), 133.7 (4-C $\left.\mathrm{Bz}_{3}\right), 148.2$ (7-C), 152.6 (11a-C), 164.9 (6-C), $169.2(\mathrm{COPh}), \quad 170.1 \quad\left(\mathrm{COCH}_{3}\right), \quad 170.3$ $\left(\mathrm{COCH}_{3}\right)$ ppm; IR $(\mathrm{KBr}): \bar{v}=3446,2930,1753,1731$, $1683,1652,1448,1362,1270,1239,1157,1069,1028$, $803,711 \mathrm{~cm}^{-1}$.

(-)-7-Hydroxy-6-oxo-1,2,3,4,4a,9,10,12b-octahydro[1,4]dioxino[2,3-j]phenanthridine-2,3,4-triyl triacetate [(-)-54, $\mathrm{C}_{21} \mathrm{H}_{23} \mathrm{NO}_{10}$ ] Yield: 55\%; white solid; m.p.: $154-156{ }^{\circ} \mathrm{C}$; $R_{f}=0.85$ (hexane/EtOAc, 1:2); ${ }^{1} \mathrm{H}$ NMR $\quad(300 \mathrm{MHz}$, $\left.\mathrm{CDCl}_{3}\right): \delta=12.54(\mathrm{~s}, 1 \mathrm{H}, \mathrm{OH}), 6.25(\mathrm{~s}, 1 \mathrm{H}, 12-\mathrm{H}), 5.95(\mathrm{~s}$, $1 \mathrm{H}, \mathrm{NH}), 5.44(\mathrm{t}, J=3.0 \mathrm{~Hz}, 1 \mathrm{H}, 3-\mathrm{H}), 5.20-5.16(\mathrm{~m}, 2 \mathrm{H}$, $2-\mathrm{H}$ and 4-H), $4.32\left(\mathrm{~s}, 4 \mathrm{H}, \mathrm{OCH}_{2} \mathrm{CH}_{2} \mathrm{O}\right), 3.76(\mathrm{dd}$, $J=12.6,11.1 \mathrm{~Hz}, 1 \mathrm{H}, 4 \mathrm{a}-\mathrm{H}), 3.10(\mathrm{td}, J=13.2,3.3 \mathrm{~Hz}$, 1H, 12b-H), 2.43 (dt, $\left.J=14.4,2.7 \mathrm{~Hz}, 1 \mathrm{H}, 1-\mathrm{H}_{\alpha}\right), 2.13$ (s, $\left.3 \mathrm{H}, \mathrm{COCH}_{3}\right), 2.09\left(\mathrm{~s}, 3 \mathrm{H}, \mathrm{COCH}_{3}\right), 2.08\left(\mathrm{~s}, 3 \mathrm{H}, \mathrm{COCH}_{3}\right)$, 1.90 (ddd, $J=14.4,12.6,2.7 \mathrm{~Hz}, 1 \mathrm{H}, 1-\mathrm{H}_{\beta}$ ) ppm; ${ }^{13} \mathrm{C}$ NMR $\left(75 \mathrm{MHz}, \quad \mathrm{CDCl}_{3}\right): \quad \delta=20.7 \quad\left(\mathrm{COCH}_{3}\right), \quad 20.8$ $\left(\mathrm{COCH}_{3}\right), 21.0\left(\mathrm{COCH}_{3}\right), 26.4$ (1-C), 34.1 (12b-C), 52.8 (4a-C), $64.1\left(\mathrm{OCH}_{2} \mathrm{CH}_{2} \mathrm{O}\right), 64.8\left(\mathrm{OCH}_{2} \mathrm{CH}_{2} \mathrm{O}\right), 67.3(3-\mathrm{C})$, 68.5 (2-C), 71.8 (4-C), 103.7 (12-C), 104.5 (6a-C), 131.1 (7a-C), 132.2 (12a-C), 148.2 (7-C), 152.6 (11a-C), 169.1 (6-C), $169.3\left(\mathrm{COCH}_{3}\right), 170.1\left(\mathrm{COCH}_{3}\right), 170.3\left(\mathrm{COCH}_{3}\right)$ ppm; IR (KBr): $\bar{v}=3337,2937,1752,1652,1586,1447$, $1370,1246,1225,1125,1056,1035,858 \mathrm{~cm}^{-1} ;[\alpha]_{\mathrm{D}}^{22}=$ $-58.0^{\circ}\left(c=0.5, \mathrm{CHCl}_{3}\right)$.

( \pm )-2-Benzoyloxy-11-methoxy-6-oxo-1,2,3,4,4a,8,9,12b-octahydro[1,4]dioxino[2,3-i]phenanthridine-3,4-diyl diacetate [(土)-46, $\left.\mathrm{C}_{27} \mathrm{H}_{27} \mathrm{NO}_{10}\right]$ Yield: $18 \%$; white solid (fluffy); m.p.: $237-238{ }^{\circ} \mathrm{C} ; \quad R_{f}=0.51$ (hexane/EtOAc, $1: 2$ ); ${ }^{1} \mathrm{H}$ NMR $\left(300 \mathrm{MHz}, \mathrm{CDCl}_{3}\right): \delta=8.06(\mathrm{dd}, J=8.1,0.9 \mathrm{~Hz}$, $2 \mathrm{H}, 2-\mathrm{H}_{\mathrm{Bz}}$ and $\left.6-\mathrm{H}_{\mathrm{Bz}}\right), 7.60(\mathrm{tt}, J=7.5,1.2 \mathrm{~Hz}, 1 \mathrm{H}, 4-$ $\left.\mathrm{H}_{\mathrm{Bz}}\right), 7.47\left(\mathrm{t}, J=7.5 \mathrm{~Hz}, 2 \mathrm{H}, 3-\mathrm{H}_{\mathrm{Bz}}\right.$ and $\left.5-\mathrm{H}_{\mathrm{Bz}}\right), 7.08(\mathrm{~s}$, $\left.1 \mathrm{H}, 12-\mathrm{H}_{\mathrm{Ar}}\right), 6.37(\mathrm{~s}, 1 \mathrm{H}, \mathrm{NH}), 5.59(\mathrm{t}, J=3.0 \mathrm{~Hz}, 1 \mathrm{H}, 3-$ H), $5.45(\mathrm{q}, J=3.0 \mathrm{~Hz}, 1 \mathrm{H}, 2-\mathrm{H}), 5.36(\mathrm{dd}, J=10.8$, $3.0 \mathrm{~Hz}, 1 \mathrm{H}, 4-\mathrm{H}), 4.54-4.50$ (m, $\left.1 \mathrm{H}, \mathrm{OHCHCH}_{2} \mathrm{O}\right), 4.39$ $4.23\left(\mathrm{~m}, 3 \mathrm{H}, \mathrm{OHCHCH} \mathrm{H}_{2} \mathrm{O}\right), 3.92\left(\mathrm{~s}, 3 \mathrm{H}, \mathrm{OCH}_{3}\right), 3.77$ (dd, $J=12.0,11.4 \mathrm{~Hz}, 1 \mathrm{H}, 4 \mathrm{a}-\mathrm{H}), 3.19(\mathrm{td}, J=12.0,3.0 \mathrm{~Hz}$, $1 \mathrm{H}, 12 \mathrm{~b}-\mathrm{H}), 2.64\left(\mathrm{dt}, J=12.6,3.0 \mathrm{~Hz}, 1 \mathrm{H}, 1-\mathrm{H}_{\alpha}\right), 2.11(\mathrm{~s}$, $\left.6 \mathrm{H}, 2 \times \mathrm{COCH}_{3}\right), 2.07-1.97\left(\mathrm{~m}, 1 \mathrm{H}, 1-\mathrm{H}_{\beta}\right) \mathrm{ppm} ;{ }^{13} \mathrm{C}$ NMR $\left(75 \mathrm{MHz}, \quad \mathrm{CDCl}_{3}\right): \quad \delta=20.7 \quad\left(\mathrm{COCH}_{3}\right), \quad 20.8$ $\left(\mathrm{COCH}_{3}\right), 27.0$ (1-C), 35.5 (12b-C), 52.1 (4a-C), 56.2 $\left(\mathrm{OCH}_{3}\right), 63.9\left(\mathrm{OCH}_{2} \mathrm{CH}_{2} \mathrm{O}\right), 64.6\left(\mathrm{OCH}_{2} \mathrm{CH}_{2} \mathrm{O}\right), 67.7$ (2C), 69.2 (4-C), 71.5 (3-C), 99.2 (6a-C), 110.6 (12-C), 128.7 $\left(3-\mathrm{C}_{\mathrm{Bz}}\right.$ and $\left.5-\mathrm{C}_{\mathrm{Bz}}\right), 129.0\left(1-\mathrm{C}_{\mathrm{Bz}}\right), 129.8\left(2-\mathrm{C}_{\mathrm{Bz}}\right.$ and $\left.6-\mathrm{C}_{\mathrm{Bz}}\right)$, 132.5 (10a-C), 133.7 (4-C $\mathrm{Bz}_{\mathrm{Bz}}$ ), 134.2 (12a-C), 145.5 (6b-C), 151.7 (11-C), 165.0 (6-C), $165.1(\mathrm{COPh}), 169.2\left(\mathrm{COCH}_{3}\right)$, $170.4\left(\mathrm{COCH}_{3}\right)$ ppm; IR $(\mathrm{KBr}): \bar{v}=3179,3078,2935$, 1753, 1723, 1668, 1598, 1495, 1451, 1369, 1330, 1269, $1238,1137,1097,1060,715 \mathrm{~cm}^{-1}$. 
(士)-3-Acetamido-6-benzoyloxy-4-(8'-methoxy-7'-methoxycarbonyl-2,3-dihydrobenzo[b][1,4]dioxin-6'-yl)cyclohexane1,2-diyl diacetate $\left[( \pm)-48, \mathrm{C}_{30} \mathrm{H}_{33} \mathrm{NO}_{12}\right]$ Yield: $8 \%$; white solid; m.p.: $244-245{ }^{\circ} \mathrm{C} ; R_{f}=0.48\left(\mathrm{CHCl}_{3} /\right.$ acetone, $\left.20: 1\right)$; ${ }^{1} \mathrm{H}$ NMR $\left(300 \mathrm{MHz}, \mathrm{CDCl}_{3}\right): \delta=8.05$ (dd, $J=7.8$, $1.2 \mathrm{~Hz}, 2 \mathrm{H}, 2-\mathrm{H}_{\mathrm{Bz}}$ and $\left.6-\mathrm{H}_{\mathrm{Bz}}\right), 7.61(\mathrm{tt}, J=7.5,1.2 \mathrm{~Hz}$, $\left.1 \mathrm{H}, 4-\mathrm{H}_{\mathrm{Bz}}\right), 7.49\left(\mathrm{t}, J=7.2 \mathrm{~Hz}, 2 \mathrm{H}, 3-\mathrm{H}_{\mathrm{Bz}}\right.$ and $\left.5-\mathrm{H}_{\mathrm{Bz}}\right), 6.72$ $\left(\mathrm{s}, 1 \mathrm{H}, 5-\mathrm{H}_{\mathrm{Ar}}\right), 6.17(\mathrm{~d}, J=9.6 \mathrm{~Hz}, 1 \mathrm{H}, \mathrm{NH}), 5.46(\mathrm{t}$, $\left.J=2.7 \mathrm{~Hz}, 1 \mathrm{H}, 2-\mathrm{H}_{\mathrm{Cy}}\right), 5.29(\mathrm{q}, J=3.0 \mathrm{~Hz}, 1 \mathrm{H}, 1-\mathrm{H})$, $5.22\left(\mathrm{dd}, \quad J=10.8, \quad 3.0 \mathrm{~Hz}, \quad 1 \mathrm{H}, 3-\mathrm{H}_{\mathrm{Cy}}\right), 4.49$ (q, $J=10.5 \mathrm{~Hz}, 1 \mathrm{H}, 4-\mathrm{H}), 4.34-4.23\left(\mathrm{~m}, 4 \mathrm{H}, \mathrm{OCH}_{2} \mathrm{CH}_{2} \mathrm{O}\right)$, $3.81\left(\mathrm{~s}, 3 \mathrm{H}, \mathrm{Ar}-\mathrm{OCH}_{3}\right), 3.60\left(\mathrm{~s}, 3 \mathrm{H}, \mathrm{COOCH}_{3}\right), 2.97$ (td, $J=12.0,4.2 \mathrm{~Hz}, 1 \mathrm{H}, 5-\mathrm{H}), 2.25\left(\mathrm{~s}, 3 \mathrm{H}, 2-\mathrm{CHOCOCH}_{3}\right.$ ), 2.25-2.21 (m, 1H, 6- $\left.\mathrm{H}_{\alpha}\right), 2.17-2.13\left(\mathrm{~m}, 1 \mathrm{H}, 6-\mathrm{H}_{\beta}\right), 2.01(\mathrm{~s}$, 3H, 3-CHOCOCH 3 ), 1.75 (s, 3H, $\left.\mathrm{NHCOCH}_{3}\right) \mathrm{ppm} ;{ }^{13} \mathrm{C}$ NMR $\left(75 \mathrm{MHz}, \mathrm{CDCl}_{3}\right): \delta=20.8\left(3-\mathrm{CHOCOCH}_{3}\right), 21.1$ $\left(2-\mathrm{CHOCOCH}_{3}\right), 23.1\left(\mathrm{NHCOCH}_{3}\right), 32.4$ (6-C), 38.8 (5C), 51.5 (4-C), $52.2\left(\mathrm{COOCH}_{3}\right), 61.5\left(\mathrm{Ar}-\mathrm{OCH}_{3}\right), 64.1$ $\left(\mathrm{OCH}_{2} \mathrm{CH}_{2} \mathrm{O}\right), 64.3\left(\mathrm{OCH}_{2} \mathrm{CH}_{2} \mathrm{O}\right), 69.0\left(2-\mathrm{C}_{\mathrm{Cy}}\right), 69.4$ (1C), $72.0\left(3-\mathrm{C}_{\mathrm{Cy}}\right), 110.8\left(5-\mathrm{C}_{\mathrm{Ar}}\right), 121.3\left(7-\mathrm{C}_{\mathrm{Ar}}\right), 128.6$ (3$\mathrm{C}_{\mathrm{Bz}}$ and 5- $\left.\mathrm{C}_{\mathrm{Bz}}\right), 129.4\left(1-\mathrm{C}_{\mathrm{Bz}}\right), 129.7\left(2-\mathrm{C}_{\mathrm{Bz}}\right.$ and $\left.6-\mathrm{C}_{\mathrm{Bz}}\right)$, $130.3\left(6-\mathrm{C}_{\mathrm{Ar}}\right), 133.6\left(4-\mathrm{C}_{\mathrm{Bz}}\right), 135.9\left(8 \mathrm{a}-\mathrm{C}_{\mathrm{Ar}}\right), 146.0\left(8-\mathrm{C}_{\mathrm{Ar}}\right)$ $146.2\left(4 \mathrm{a}-\mathrm{C}_{\mathrm{Ar}}\right), 164.6(\mathrm{OCOPh}), 169.1\left(\mathrm{COOCH}_{3}\right), 169.6$ $\left(2-\mathrm{CHOCOCH}_{3}\right), \quad 170.0 \quad\left(\mathrm{NHCOCH}_{3}\right), \quad 170.8$ $\mathrm{CHOCOCH}_{3}$ ) ppm; IR (KBr): $\bar{v}=3368,2951,1755,1731$, 1675, 1608, 1541, 1509, 1442, 1374, 1337, 1274, 1221, $1168,1128,1110,1069,716 \mathrm{~cm}^{-1}$.

( \pm )-3-Acetamido-6-benzoyloxy-4-(8'-methoxy-5'-methoxycarbonyl-2,3-dihydrobenzo[b][1,4]dioxin-6'-yl)cyclohexane1,2-diyl diacetate $\left[( \pm)-49, \mathrm{C}_{30} \mathrm{H}_{33} \mathrm{NO}_{12}\right]$ Yield: $13 \%$; white solid (fluffy); m.p.: $109-112{ }^{\circ} \mathrm{C} ; R_{f}=0.40\left(\mathrm{CHCl}_{3} /\right.$ acetone, 20:1); ${ }^{1} \mathrm{H}$ NMR $\left(300 \mathrm{MHz}, \mathrm{CDCl}_{3}\right.$ ): $\delta=8.07$ (dd, $J=7.8,1.2 \mathrm{~Hz}, 2 \mathrm{H}, 2-\mathrm{H}_{\mathrm{Bz}}$ and $\left.6-\mathrm{H}_{\mathrm{Bz}}\right), 7.62(\mathrm{tt}, J=7.5$, $\left.1.2 \mathrm{~Hz}, 1 \mathrm{H}, 4-\mathrm{H}_{\mathrm{Bz}}\right), 7.50\left(\mathrm{t}, J=7.2 \mathrm{~Hz}, 2 \mathrm{H}, 3-\mathrm{H}_{\mathrm{Bz}}\right.$ and $5-$ $\left.\mathrm{H}_{\mathrm{Bz}}\right), 6.54\left(\mathrm{~s}, 1 \mathrm{H}, 7-\mathrm{H}_{\mathrm{Ar}}\right), 5.83(\mathrm{~d}, J=9.9 \mathrm{~Hz}, 1 \mathrm{H}, \mathrm{NH})$, $5.48\left(\mathrm{t}, J=3.0 \mathrm{~Hz}, 1 \mathrm{H}, 2-\mathrm{H}_{\mathrm{Cy}}\right), 5.27(\mathrm{q}, J=3.0 \mathrm{~Hz}, 1 \mathrm{H}, 1-$ $\mathrm{H}), 5.25$ (dd, $J=$ overlapped and $\left.3.3 \mathrm{~Hz}, 1 \mathrm{H}, 3-\mathrm{H}_{\mathrm{Cy}}\right), 4.66$ $(\mathrm{q}, J=10.8 \mathrm{~Hz}, 1 \mathrm{H}, 4-\mathrm{H}), 4.32-4.22\left(\mathrm{~m}, 4 \mathrm{H}, \mathrm{OCH}_{2} \mathrm{CH}_{2-}\right.$ O), $3.92\left(\mathrm{~s}, 3 \mathrm{H}, \mathrm{Ar}-\mathrm{OCH}_{3}\right), 3.53\left(\mathrm{~s}, 3 \mathrm{H}, \mathrm{COOCH}_{3}\right), 3.09$ $(\mathrm{td}, \quad J=12.0,4.2 \mathrm{~Hz}, 1 \mathrm{H}, 5-\mathrm{H}), 2.26 \quad(\mathrm{~s}, 3 \mathrm{H}, \quad 2-$ $\left.\mathrm{CHOCOCH}_{3}\right), 2.26-2.22\left(\mathrm{~m}, 1 \mathrm{H}, 6-\mathrm{H}_{\alpha}\right), 2.17-2.13(\mathrm{~m}, 1 \mathrm{H}$, $\left.6-\mathrm{H}_{\beta}\right), 2.02\left(\mathrm{~s}, 3 \mathrm{H}, 3-\mathrm{CHOCOCH}_{3}\right), 1.74(\mathrm{~s}, 3 \mathrm{H}$, $\mathrm{NHCOCH}_{3}$ ) ppm; $\left.{ }^{13} \mathrm{C} \mathrm{NMR} \mathrm{(75} \mathrm{MHz}, \mathrm{CDCl}_{3}\right): \delta=20.8$ $\left(3-\mathrm{CHOCOCH}_{3}\right), \quad 21.2 \quad\left(2-\mathrm{CHOCOCH}_{3}\right), \quad 23.2$ $\left(\mathrm{NHCOCH}_{3}\right), 33.2$ (6-C), 39.2 (5-C), 50.6 (4-C), 52.2 $\left(\mathrm{COOCH}_{3}\right), 56.4\left(\mathrm{Ar}-\mathrm{OCH}_{3}\right), 64.2\left(\mathrm{OCH}_{2} \mathrm{CH}_{2} \mathrm{O}\right), 64.4$ $\left(\mathrm{OCH}_{2} \mathrm{CH}_{2} \mathrm{O}\right), 69.1\left(2-\mathrm{C}_{\mathrm{Cy}}\right), 69.5(1-\mathrm{C}), 71.8\left(3-\mathrm{C}_{\mathrm{Cy}}\right)$, $102.0\left(7-\mathrm{C}_{\mathrm{Ar}}\right), 116.2\left(5-\mathrm{C}_{\mathrm{Ar}}\right), 128.6\left(3-\mathrm{C}_{\mathrm{Bz}}\right.$ and $\left.5-\mathrm{C}_{\mathrm{Bz}}\right)$, $129.4\left(1-\mathrm{C}_{\mathrm{Bz}}\right), 129.7\left(2-\mathrm{C}_{\mathrm{Bz}}\right.$ and $\left.6-\mathrm{C}_{\mathrm{Bz}}\right), 130.3\left(6-\mathrm{C}_{\mathrm{Ar}}\right)$, $131.8\left(8 \mathrm{a}-\mathrm{C}_{\mathrm{Ar}}\right), 133.6\left(4-\mathrm{C}_{\mathrm{Bz}}\right), 141.2\left(4 \mathrm{a}-\mathrm{C}_{\mathrm{Ar}}\right), 150.2(8-$ $\left.\mathrm{C}_{\mathrm{Ar}}\right) \quad 164.6(\mathrm{OCOPh}), 168.5 \quad\left(\mathrm{COOCH}_{3}\right), 169.6 \quad(2-$ $\left.\mathrm{CHOCOCH}_{3}\right), 169.9\left(\mathrm{NHCOCH}_{3}\right), 170.7\left(3-\mathrm{CHOCOCH}_{3}\right)$ ppm; IR (KBr): $\bar{v}=2948,1750,1729,1670,1541,1493$, $1456,1371,1332,1276,1239,1160,1112,1069$, $716 \mathrm{~cm}^{-1}$.

\section{General procedure for the modified Zemplén's deacetylation}

To a solution of $84.2 \mathrm{mg}(-)-44(0.17 \mathrm{mmol}), 78.7 \mathrm{mg}$ (-)-52 (0.17 mmol), $78.7 \mathrm{mg}(-)-53 \quad(0.17 \mathrm{mmol})$, or $76.3 \mathrm{mg}(-)-54(0.17 \mathrm{mmol})$ in $12.5 \mathrm{~cm}^{3}$ anhydrous tetrahydrofuran, $6.50 \mathrm{~cm}^{3} 0.53 \mathrm{M}$ methanolic solution of sodium methoxide was added dropwise at $\mathrm{rt}$ and the reaction mixture was stirred also at $\mathrm{rt}$ for $2 \mathrm{~h}$. Then Amberlyte IR-120 (strongly acidic resin) was added until the $\mathrm{pH}$ became 6 . The solid resin was filtered and washed with $10 \mathrm{~cm}^{3}$ methanol, and then the filtrate was concentrated in vacuo. The crude product was purified by preparative TLC (EtOAc/ethanol, 6:1) to afford (-)-8, (-)-9, (-)-10, or (-)-11.

(-)-2,3,4-Trihydroxy-1,3,4,4a,5,9,10,12b-octahydro[1,4]dioxino[2,3-j]phenanthridin-6(2H)-one $\left[(-)-8, \mathrm{C}_{15} \mathrm{H}_{17} \mathrm{NO}_{6}\right]$ Yield: $71 \%$; white solid (fluffy); m.p.: $162{ }^{\circ} \mathrm{C} ; R_{f}=0.52$ (EtOAc/methanol, 20:1); ${ }^{1} \mathrm{H}$ NMR (300 MHz, DMSO- $\left.d_{6}\right)$ : $\delta=7.30$ (s, 1H, NH), 6.90 (s, 1H, 7-H), 6.77 (s, 1H, 12-H), 5.17-4.93 (m, 2H, $2 \times \mathrm{OH}), 4.93-4.79(\mathrm{~m}, 1 \mathrm{H}, \mathrm{OH}), 4.29$ $4.24\left(\mathrm{~m}, 4 \mathrm{H}, \mathrm{OCH}_{2} \mathrm{CH}_{2} \mathrm{O}\right), 3.91-3.84(\mathrm{~m}, 1 \mathrm{H}, 2-\mathrm{H}), 3.72-$ $3.68(\mathrm{~m}, 2 \mathrm{H}, 3-\mathrm{H}$ and 4-H), $2.86(\mathrm{td}, J=11.7,3.0 \mathrm{~Hz}, 1 \mathrm{H}$, 12b-H), 2.09 (dt, $J=13.5,3.0 \mathrm{~Hz}, 1 \mathrm{H}, 1-\mathrm{H}_{\alpha}$ ), 1.63 (ddd, $\left.J=14.7, \quad 11.7, \quad 2.1 \mathrm{~Hz}, \quad 1 \mathrm{H}, \quad 1-\mathrm{H}_{\beta}\right) \quad \mathrm{ppm} ;{ }^{13} \mathrm{C} \quad \mathrm{NMR}$ (75 MHz, DMSO- $\left.d_{6}\right): \delta=28.7$ (1-C), 34.2 (12b-C), 55.7 (4a-C), $64.4\left(\mathrm{OCH}_{2} \mathrm{CH}_{2} \mathrm{O}\right), 64.9\left(\mathrm{OCH}_{2} \mathrm{CH}_{2} \mathrm{O}\right), 69.1$ (2-C), 70.2 (4-C), 72.2 (3-C), 112.8 (7-C), 116.5 (12-C), 123.1 (6a-C), 136.4 (12a-C), 142.3 (7a-C), 147.1 (11a-C), 164.7 (6-C) ppm; IR (KBr): $\bar{v}=3446,2910,1716,1683,1580$, 1509, 1473, 1374, 1315, 1225, 1148, 1076, 1034, 912, 889, $790 \mathrm{~cm}^{-1} ;[\alpha]_{\mathrm{D}}^{22}=-26.7^{\circ}(c=1$, methanol); $e e>99 \%$.

(-)-2,3,4,7-Tetrahydroxy-1,2,3,4,4a,9,10,12b-octahydro[1,4]dioxino[2,3-j]phenanthridin-6(2H)-one [(-)-9, $\left.\mathrm{C}_{15} \mathrm{H}_{17} \mathrm{NO}_{7}\right]$ Yield: 76\%; white solid; m.p.: $174-176{ }^{\circ} \mathrm{C}$; $R_{f}=0.76$ (EtOAc/methanol, 4:1); ${ }^{1} \mathrm{H}$ NMR $(300 \mathrm{MHz}$, DMSO- $\left.d_{6}\right): \delta=13.0(\mathrm{~s}, 1 \mathrm{H}, \mathrm{OH}), 7.45(\mathrm{~s}, 1 \mathrm{H}, \mathrm{NH}), 6.25$ (s, 1H, 12-H), 5.01-4.92 (m, 2H, $2 \times \mathrm{OH}), 4.89-4.82(\mathrm{~m}$, $1 \mathrm{H}, \mathrm{OH}), 4.28-4.22\left(\mathrm{~m}, 4 \mathrm{H}, \mathrm{OCH}_{2} \mathrm{CH}_{2} \mathrm{O}\right), 3.91-3.83(\mathrm{~m}$, $1 \mathrm{H}, 2-\mathrm{H}$ or $3-\mathrm{H}$ or $4-\mathrm{H}), 3.77-3.65(\mathrm{~m}, 2 \mathrm{H}, 2 \times 2-\mathrm{H}$ or $3-\mathrm{H}$ or $4-\mathrm{H}), 2.81(\mathrm{td}, J=12.3,3.3 \mathrm{~Hz}, 1 \mathrm{H}, 12 \mathrm{~b}-\mathrm{H}), 2.10-1.98$ $\left(\mathrm{m}, 1 \mathrm{H}, 1-\mathrm{H}_{\alpha}\right), 1.66-1.56\left(\mathrm{~m}, 1 \mathrm{H}, 1-\mathrm{H}_{\beta}\right) \mathrm{ppm}$ (the sign of $4 \mathrm{a}-\mathrm{H}$ is covered by that of water in DMSO- $\left.d_{6}\right) ;{ }^{13} \mathrm{C} \mathrm{NMR}$ (75 MHz, DMSO- $\left.d_{6}\right): \delta=27.8$ (1-C), 33.1 (12b-C), 55.1 (4a-C), $63.4\left(\mathrm{OCH}_{2} \mathrm{CH}_{2} \mathrm{O}\right), 64.4\left(\mathrm{OCH}_{2} \mathrm{CH}_{2} \mathrm{O}\right), 68.4$ (2-C), 69.5 (4-C), 71.6 (3-C), 102.8 (12-C), 104.4 (6a-C), 130.1 (8a-C), 134.4 (12a-C), 147.5 (7-C), 151.5 (11a-C), 169.7 (6-C) ppm; IR (KBr): $\bar{v}=3421,2926,1647,1626,1587$, 
$1448,1400,1362,1281,1230,1126,1064,1030,910$, $811 \mathrm{~cm}^{-1} ;[\alpha]_{\mathrm{D}}^{22}=-18.5^{\circ}(c=0.13$, ethanol $) ; e e>99 \%$.

\section{(-)-2,3,4-Trihydroxy-7-methoxy-1,2,3,4,4a,9,10,12b-octahy-} dro[1,4]dioxino[2,3-j]phenanthridin-6(2H)-one [(-)-10, $\left.\mathrm{C}_{16} \mathrm{H}_{19} \mathrm{NO}_{7}\right]$ Yield: $47 \%$; white solid; m.p.: $163-168{ }^{\circ} \mathrm{C}$; $R_{f}=0.39$ (EtOAc/methanol, 4:1); ${ }^{1} \mathrm{H}$ NMR $(300 \mathrm{MHz}$, DMSO- $\left.d_{6}\right): \delta=6.91(\mathrm{~s}, 1 \mathrm{H}, \mathrm{NH}), 6.53(\mathrm{~s}, 1 \mathrm{H}, 12-\mathrm{H}), 5.10-$ $4.91(\mathrm{~m}, 1 \mathrm{H}, \mathrm{OH}), 4.91-4.67(\mathrm{~m}, 2 \mathrm{H}, 2 \times \mathrm{OH}), 4.31-4.21$ $\left(\mathrm{m}, 4 \mathrm{H}, \mathrm{OCH}_{2} \mathrm{CH}_{2} \mathrm{O}\right), 3.89-3.84(\mathrm{~m}, 1 \mathrm{H}, 2-\mathrm{H}$ or $3-\mathrm{OH}$ or $4-\mathrm{H}), 3.74\left(\mathrm{~s}, 3 \mathrm{H}, \mathrm{OCH}_{3}\right), 3.72-3.65(\mathrm{~m}, 2 \mathrm{H}, 2 \times 2-\mathrm{H}$ or $3-$ $\mathrm{OH}$ or $4-\mathrm{H}), 3.17(\mathrm{dd}, J=11.1,10.5 \mathrm{~Hz}, 1 \mathrm{H}, 4 \mathrm{a}-\mathrm{H}), 2.74$ $(\mathrm{td}, J=12.6,3.6 \mathrm{~Hz}, 1 \mathrm{H}, 12 \mathrm{~b}-\mathrm{H}), 2.03$ (dt, $J=12.9$, $\left.2.7 \mathrm{~Hz}, 1 \mathrm{H}, 1-\mathrm{H}_{\alpha}\right), 1.63-1.53\left(\mathrm{~m}, 1 \mathrm{H}, 1-\mathrm{H}_{\beta}\right) \mathrm{ppm} ;{ }^{13} \mathrm{C}$ NMR (75 MHz, DMSO- $\left.d_{6}\right): \delta=28.3$ (1-C), 34.8 (12b-C), $54.5 \quad(4 \mathrm{a}-\mathrm{C}), 60.9 \quad\left(\mathrm{OCH}_{3}\right), 63.6\left(\mathrm{OCH}_{2} \mathrm{CH}_{2} \mathrm{O}\right), 64.2$ $\left(\mathrm{OCH}_{2} \mathrm{CH}_{2} \mathrm{O}\right), 68.5$ (2-C), 69.4 (4-C), 71.5 (3-C), 107.3 (12-C), 116.1 (6a-C), 135.9 (10a-C), 136.1 (12a-C), 146.5 (6b-C), 149.4 (11-C), 162.6 (6-C) ppm; IR (KBr): $\bar{v}=3392,2927,1717,1652,1475,1331,1226,1122,1068$, $1039,910 \mathrm{~cm}^{-1} ; \quad[\alpha]_{\mathrm{D}}^{22}=-34.5^{\circ} \quad(c=0.63$, ethanol $)$; $e e>99 \%$.

\section{(-)-2,3,4-Trihydroxy-11-methoxy-1,2,3,4,4a,8,9,12b-octahy-} dro[1,4]dioxino[2,3-i]phenanthridin-6(2H)-one [(-)-11, $\left.\mathrm{C}_{16} \mathrm{H}_{19} \mathrm{NO}_{7}\right] \quad$ Yield: $47 \%$; white solid; m.p.: $204-207{ }^{\circ} \mathrm{C}$; $R_{f}=0.20$ (EtOAc/methanol, 4:1); ${ }^{1} \mathrm{H}$ NMR $(300 \mathrm{MHz}$, DMSO- $\left.d_{6}\right): \delta=6.76(\mathrm{~s}, 1 \mathrm{H}, \mathrm{NH}), 6.45(\mathrm{~s}, 1 \mathrm{H}, 12-\mathrm{H}), 4.96$ $(\mathrm{d}, J=3.3 \mathrm{~Hz}, 1 \mathrm{H}, \mathrm{OH}), 4.89(\mathrm{~d}, J=6.3 \mathrm{~Hz}, 1 \mathrm{H}, \mathrm{OH})$, $4.78(\mathrm{~d}, J=2.4 \mathrm{~Hz}, 1 \mathrm{H}, \mathrm{OH}), 4.31-4.11\left(\mathrm{~m}, 4 \mathrm{H}, \mathrm{OCH}_{2-}\right.$ $\left.\mathrm{CH}_{2} \mathrm{O}\right), 3.91-3.86(\mathrm{~m}, 1 \mathrm{H}, 2-\mathrm{H}$ or $3-\mathrm{H}$ or $4-\mathrm{H}), 3.81(\mathrm{~s}, 3 \mathrm{H}$, $\left.\mathrm{OCH}_{3}\right), 3.73-3.65(\mathrm{~m}, 2 \mathrm{H}, 2 \times 2 \mathrm{H}$ or $3-\mathrm{H}$ or $4-\mathrm{H}), 3.19$ (dd, $J=11.4,10.2 \mathrm{~Hz}, 1 \mathrm{H}, 4 \mathrm{a}-\mathrm{H}), 2.77$ (td, $J=12.6$, $3.3 \mathrm{~Hz}, 1 \mathrm{H}, 12 \mathrm{~b}-\mathrm{H}), 2.13\left(\mathrm{dt}, J=13.2,3.3 \mathrm{~Hz}, 1 \mathrm{H}, 1-\mathrm{H}_{\alpha}\right.$ ), $1.69-1.60\left(\mathrm{~m}, 1 \mathrm{H}, 1-\mathrm{H}_{\beta}\right) \mathrm{ppm} ;{ }^{13} \mathrm{C}$ NMR $(75 \mathrm{MHz}$, DMSO- $\left.d_{6}\right): \delta=28.3$ (1-C), 35.0 (12b-C), 54.5 (4a-C), 55.6 $\left(\mathrm{OCH}_{3}\right), 63.0\left(\mathrm{OCH}_{2} \mathrm{CH}_{2} \mathrm{O}\right), 63.7\left(\mathrm{OCH}_{2} \mathrm{CH}_{2} \mathrm{O}\right), 68.6$ (2C), 69.6 (4-C), 71.6 (3-C), 99.3 (12-C), 111.0 (6a-C), 131.5 (10a-C), 136.1 (12a-C), 144.5 (6b-C), 150.5 (11-C), 162.7 (6-C) ppm; IR (KBr): $\bar{v}=3399,2923,1648,1600,1495$, $1455,13631329,1131,1068,900 \mathrm{~cm}^{-1} ;[\alpha]_{\mathrm{D}}^{22}=-66.5^{\circ}$ ( $c=0.25$, ethanol); $e e>99 \%$.

\section{References}

1. Kornienko A, Evidente A (2008) Chem Rev 108:1982

2. Ghosal S, Saini KS, Razdan S (1985) Phytochemistry 10:2141

3. Gerrard AW (1877) Pharm J 8:214

4. Cook JW, Loudon JD (1952) Alkaloids of the amaryllidaceae. In: Manske RHF, Holmes HL (eds) The alkaloids: chemistry and physiology, vol 2. Academic Press, New York, p 331

5. Pettit GR, Cragg GM, Singh SB, Duke JA, Doubek DL (1990) J Nat Prod 53:176
6. Pettit GR, Pettit GR III, Backhaus RA, Boyd MR, Meerow AW (1993) J Nat Prod 56:1682

7. Banwell MG, Cowden CJ, Gable RW (1994) J Chem Soc Perkin Trans 1:3515

8. McNulty J, Mo R (1998) Chem Commun:933

9. McNulty J, Mao J, Gibe R, Mo R, Wolf S, Pettit GR, Herald DL, Boyd MR (2001) Bioorg Med Chem Lett 11:169

10. McNulty J, Larichev V, Pandey S (2005) Bioorg Med Chem Lett 15:5315

11. Hudlicky T, Rinner U, Gonzalez D, Akgun H, Schilling S, Siengalewicz P, Martinot TA, Pettit GR (2002) J Org Chem 67:8726

12. Shukla KH, Boehmler DJ, Bogacyzk S, Duvall BR, Peterson WA, McElroy WT, DeShong P (2006) Org Lett 8:4183

13. Ortiz JC, Ozores L, Cagide-Fagín F, Alonso R (2006) Chem Commun: 4239

14. Matveenko M, Kokas OJ, Banwell MG, Willis AC (2007) Org Lett 9:3683

15. Metveenko M, Banwell MG, Joffe M, Wan S, Fantino E (2009) Chem Biodiversity 6:685

16. Collins J, Drouin M, Sun X, Rinner U, Hudlicky T (2008) Org Lett 10:361

17. Manpadi M, Kireev AS, Magedov IV, Altig J, Tongwa P, Antipin MY, Evidente A, van Otterlo WAL, Kornienko A (2009) J Org Chem $74: 7122$

18. Collins J, Rinner U, Moser M, Hudlicky T, Ghiviriga I, Romero AE, Kornienko A, Ma D, Griffin C, Pandey S (2010) J Org Chem 75:3069

19. Vshyvenko S, Scattolon J, Hudlicky T, Romero AE, Kornienko A (2011) Bioorg Med Chem Lett 21:4750

20. Vshyvenko S, Scattolon J, Hudlicky T, Romero AE, Kornienko A, Ma D, Tuffley I, Pandey S (2012) Can J Chem 90:932

21. Rinner U, Hillebrenner HL, Adams DR, Hudlicky T, Pettit GR (2004) Bioorg Med Chem Lett 14:2911

22. Rinner U, Hudlicky T, Gordon H, Pettit GR (2004) Angew Chem Int Ed 43:5342

23. Moser M, Sun T, Hudlicky T (2005) Org Lett 75:669

24. Afarinkia K, Ndibwami A (2007) Synlett 2007:1940

25. de La Sovera V, Bellomo A, Gonzalez D (2011) Tetrahedron Lett 52:430

26. Nieto-García O, Alonso R (2013) Org Biomol Chem 11:515

27. Vshyvenko S, Reisenauer MR, Rogelj S, Hudlicky T (2014) Bioorg Med Chem Lett 24:4236

28. Vshyvenko S, W'Giorgis Z, Weber A, Neverova N, Hedberg B, Hudlicky T (2015) Adv Synth Catal 357:83

29. McNulty J, D'Aiuto L, Zhi Y, McClain L, Zepeda-Velázquez C, Ler S, Jenkins HA, Yee MB, Piazza P, Yolken RH, Kinchington PR, Nimgaonkar VL (2016) ACS Med Chem Lett 7:46

30. He J, Qi W-B, Wang L, Tian J, Jiao P-R, Liu G-Q, Ye W-C, Liao M (2013) Influenza Other Respir Viruses 7:922

31. Li S, Chen C, Zhang H, Guo H, Wang H, Wang L, Zhang X, Hua S, Yu J, Xiao P, Li R, Tan X (2005) Antivir Res 67:18

32. Szlávik L, Gyuris Á, Minárovits J, Forgó P, Molnár J, Hohmann J (2004) Planta Med 70:871

33. Gabrielsen B, Monath TP, Huggins JW, Kefauver DF, Pettit GR, Groszek G, Hollingshead M, Kirsi JJ, Shannon WM, Schubert EM, DaRe J, Ugarkar B, Ussery MA, Phelan MJ (1992) J Nat Prod 55:1569

34. Gabrielsen B, Monath TP, Huggins JW, Kirsi JJ, Hollingshead M, Shannon WM, Pettit GR (1992) Activity of selected amaryllidaceae constituents and related synthetic substances against medically important RNA viruses. In: Chu CK, Cutler HG (eds), Natural Products as Antiviral Agents, Springer, US, New York, p 121

35. Revu O, Zepeda-Velázquez $\mathrm{C}$, Nielsen AJ, McNulty J, Yolken RH, Jones-Brando L (2016) ChemistrySelect 1:5895 
36. Szántó G, Hegedűs L, Mattyasovszky L, Simon A, Simon Á, Kádas I (2009) Tetrahedron Lett 50:2857

37. Szántó G, Hegedűs L, Mattyasovszky L, Simon A, Simon Á, Bitter I, Tóth G, Tőke L, Kádas I (2009) Tetrahedron 65:8412

38. Varró G, Hegedűs L, Simon A, Kádas I (2016) Tetrahedron Lett $57: 1544$

39. Varró G, Hegedűs L, Simon A, Balogh A, Grün A, Leveles I, Vértessy BG, Kádas I (2017) J Nat Prod 80:1909

40. Varró G, Mattyasovszky L, Grün A, Simon A, Hegedűs L, Kádas I (2018) Synthesis 50:625

41. Lange RG (1962) J Org Chem 27:2037

42. Smith P (1958) J Chem Soc: 3740

43. Altemöller M, Gehring T, Cudaj J, Podlech J, Goessmann H, Feldmann C, Rothenberger A (2009) Eur J Org Chem 2009:2130

44. Leblond B, Taverne T, Beausoleil E, Chauvignac C, Casagrande A-S, Desire L (2011) Substituted isoquinolines and their use as tubulin polymerization inhibitors. PCT Int. Patent Appl. WO 2011151423, June 1 2011; (2011) Chem Abstr 156:35001

45. Peseke K, Götze L, Reinke H, Cedeño QA, Suarez JQ, Andreu MG, Castro HV (1997) J Prakt Chem/Chem-Ztg 339:656

46. Brunner H, Bugler J, Nuber B (1995) Tetrahedron: Asymmetry 6:1699

47. Brunner H, Bugler J (1997) Bull Soc Chim Belg 106:77

48. Lv J, Zhang J, Lin Z, Wang Y (2009) Chem Eur J 15:972

49. Xie J-W, Chen W, Li R, Zeng M, Du W, Yue L, Chen Y-C, Wu Y, Zhu J, Deng J-G (2007) Angew Chem Int Ed 46:389
50. Dong L-T, Lu R-J, Du Q-S, Liu S-P, Xuan Y-N, Yan M (2009) Tetrahedron 65:4124

51. Liu C, Lu Y (2010) Org Lett 12:2278

52. Cui H-F, Li P, Wang X-W, Chai Z, Yang Y-Q, Cai Y-P, Zhu S-Z, Zhao G (2011) Tetrahedron 67:312

53. Liu W, Mei D, Wang W, Duan W (2013) Tetrahedron Lett $54: 3791$

54. Walker GN (1965) J Org Chem 30:1416

55. Szántó G, Kádas I, Kárpáti T, Hegedüs L (2010) Reac Kinet Mech Cat 99:85

56. Máthé T, Tungler A, Petró J (1982) Process for the preparation of supported metal catalysts. US Patent 4,361,500, Nov 30 1982; (1983) Chem Abstr 98:78874

57. Fuji H, Oshima K, Utimoto K (1991) Chem Lett 20:1847

58. Shull BK, Sakai T, Nichols JB, Koreeda M (1997) J Org Chem 62:8294

59. VanRheenen V, Kelly RC, Cha DY (1976) Tetrahedron Lett 17:1973

60. Banwell MG, Bissett BD, Busato S, Cowden CJ, Hockless DCR, Holman JW, Read RW, Wu AW (1995) J Chem Soc Chem Commun:2551

61. Zemplén G, Pacsu E (1929) Ber Dtsch Chem Ges 62:1613

62. Pouységu L, Sylla T, Garnier T, Rojas LB, Charris J, Deffieux D, Quideau S (2010) Tetrahedron 10:5908

63. Sugasawa S, Arata Y (1956) Pharm Bull 4:406

64. Czakó Z, Juhász L, Docsa T, Gergely P, Antus S (2010) Pharmazie 65:235 